\title{
Combined application of a biopesticide and a botanical compound to control fungus gnats Bradysia impatiens
}

\author{
Dissertation \\ zur Erlangung des Doktorgrades \\ der Fakultät für Agrarwissenschaften \\ der Georg-August-Universität Göttingen \\ vorgelegt von \\ Mahsa Dehghani \\ geboren am 24.04.1987 in Kerman, Iran
}

Göttingen, July 2020 
1. Referentin/Referent: Prof. Dr. Stefan Vidal

2. Korreferentin/Korreferent: Dr. sc. nat. Rainer Meyhöfer

3. Korreferentin/Korreferent: Prof. Dr. Petr Karlovsky

Tag der mündlichen Prüfung: 08.07.2020 
Table of contents

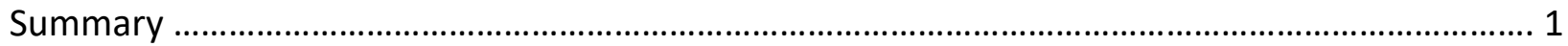

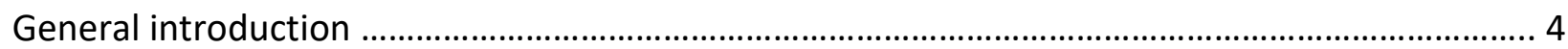

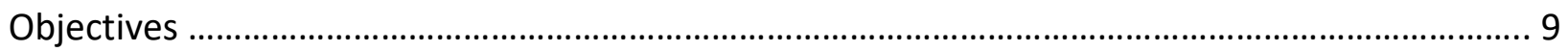

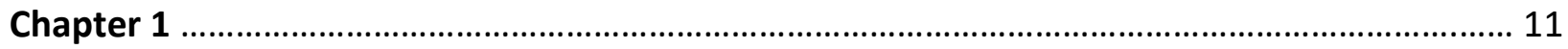

Utilization of entomopathogenic fungi, Metarhizium brunneum and Beauveria bassiana to control larvae of fungus gnats

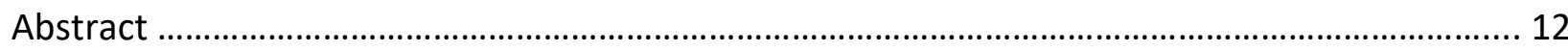

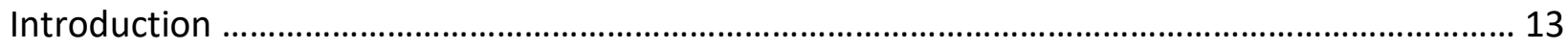

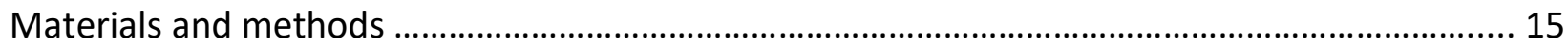

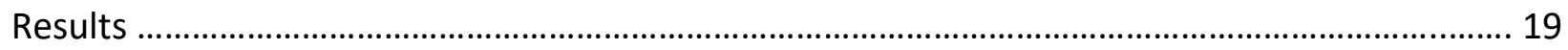

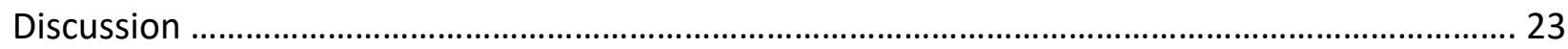

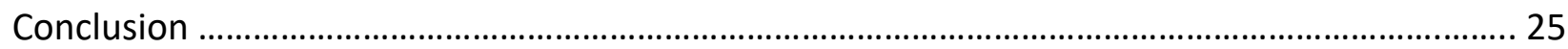

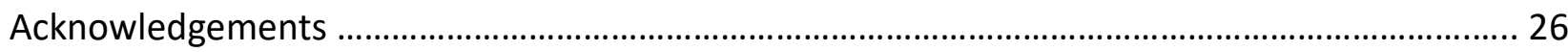

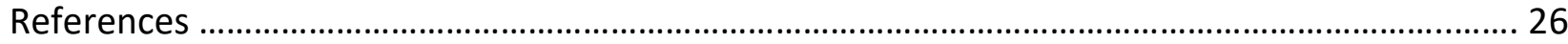

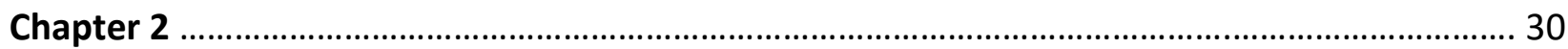

Combining a biopesticide and a botanical compound against fungus gnat larvae Bradysia impatiens (Diptera: Sciaridae)

Abstract

Introduction 
Results

Discussion 44

Conclusion

Acknowledgements

References

Chapter 3

An attract and kill formulation for fungus gnat control with combining a biopesticide and a botanical compound

Abstract 52

Introduction 53

Materials and methods 55

Results 61

Discussion 71

Conclusion 75

Acknowledgements 75

References 75

Chapter 4 83

Combined application of a biopesticide and a botanical compound to control fungus gnats Bradysia impatiens on different host plants

Abstract 


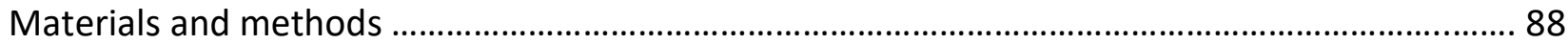

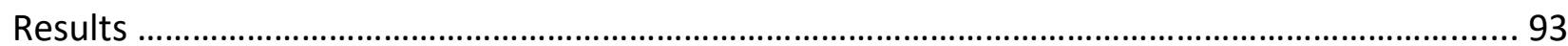

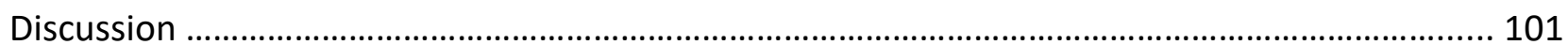

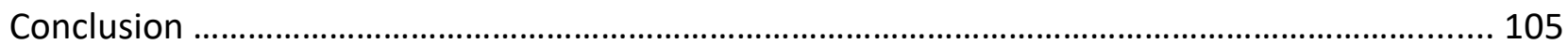

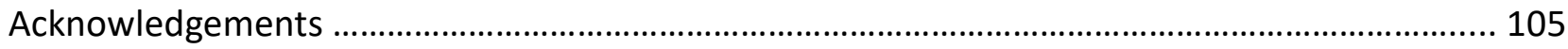

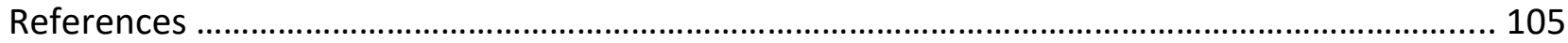

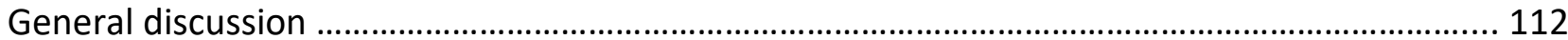

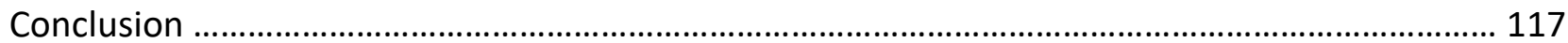

References: General introduction and general discussion ..................................................... 119

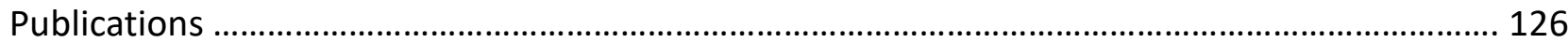

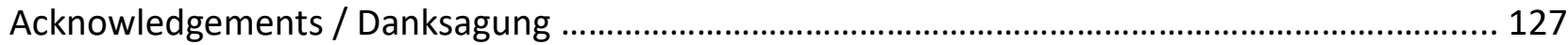




\section{Summary}

Fungus gnats, including the common species Bradysia impatiens Johannsen (Diptera: Sciaridae) are one of the most common pests in greenhouse production systems, mainly due to the direct feeding damage by larvae on the roots and stems. Environmental pollution, human health concerns and a high risk of insecticides require new, environmentally safe and effective strategies for the management of fungus gnats in greenhouses. Specifically, organic production systems need to fall back on biological control strategies for fungus gnat control. Several microbial products and biorationale insecticides have been developed and evaluated as pest control alternatives, with varying efficacies. In a first series of bioassays we evaluated the efficacy of entomopathogenic fungi on the survival rate and adult emergence of fungus gnats. In a second series of bioassays, "attract and kill" formulations for fungus gnat control by combining a biopesticide (Metarhizium brunneum $\mathrm{Cb} 15$ and Spinosad) and a botanical compound, formulated in a slow release capsule were studied. In subsequent laboratory bioassays, the application of biopesticides (M. brunneum, Bacillus thuringiensis Bt and Spinosad) alone or in combination with the botanical capsule were tested on attraction and oviposition of the fungus gnat B. impatiens. Furthermore, the effectiveness of the two biopesticides ( $M$. brunneum and Spinosad) individually or in combination with the botanical capsules were assessed against fungus gnats using three different host plants (basil, parsley and poinsettia), under greenhouse conditions. These combined formulations were assessed to improve the control efficiency of fungus gnats aiming at an appropriate practical control strategy for fungus gnat $B$. impatiens control in greenhouse production systems.

1. The effect of the entomopathogenic fungi EPF (isolates of two Metarhizium brunneum, three Beauveria bassiana and one Acremonium strictum) as pure fungal diets or as spore suspensions mixed with the substrate were studied on fungus gnat larvae $B$. impatiens.

a. Fungus gnat larvae are able to survive even on pure entomopathogenic fungal diets. Larval stages of fungus gnats were more susceptible to $M$. brunneum in comparison to other fungal strains, which resulted in a reduced number of emerging adults. Larvae fed on fungal strains B. bassiana and $A$. strictum had a high percentage of survivorship.

b. Less larvae of $B$. impatiens survived, when EPF spore suspensions were mixed with the substrate. Hence, fewer numbers of adults emerged from larvae exposed to the substrate treated with M. brunneum and A. strictum.

c. M. brunneum was therefore selected as a suitable EPF to control fungus gnat larvae. 
2. The effectiveness of two different formulations of botanical compounds containing mint oil (Mentha and Minx) and the combination of a biopesticide with these botanical compounds was assessed against larvae of $B$. impatiens.

a. Minx capsules showed an attractant effect for fungus gnat larvae.

b. The biopesticides $M$. brunneum or Bt individually reduced the emergence rate of fungus gnat adults.

c. The application of M. brunneum or Bt combined with the botanical capsules Minx resulted in a higher reduction of adult emergence, although not significantly, as compared to the single treatments interpreted as an additive effect on the efficacy of biopesticide.

d. The combined application of biopesticides ( $M$. brunneum or Bt) and the botanical capsules (Minx) points towards an "attract and kill" strategy.

3. The egg-laying behavior of fungus gnats when exposed to $M$. brunneum and two biopesticides (Bt or Spinosad) individually or in combination with Minx capsules was tested in laboratory experiments. Moreover, the effect of combining a biopesticide (M. brunneum, Neem Azal or Spinosad) and the botanical capsules (Minx) was evaluated in an "attract and kill" approach targeting fungus gnat larvae in laboratory experiments.

a. Fungus gnat females preferred to lay more eggs in Spinosad treatments individually or in combination with the botanical capsules.

b. Eggs laid by fungus gnat females in Spinosad treatments did not successfully finalize their life cycle to adults, resulting in an efficient reduction of adult emergence, either when applied alone $(99.81 \%)$ or in combination with the botanical compound $(99.93 \%)$ as compared to the other treatments. Hence, Spinosad in combination with Minx capsules might be used as a "trap crop strategy".

c. Fungus gnat larvae showed a positive interaction with the Minx capsules. Moreover, fungus gnat females exhibited a strong ovipositional preference for the substrate treated with the botanical capsules vs. the control substrate.

d. The combination of biopesticides (M. brunneum and Spinosad) with the Minx capsules, specifically Spinosad, resulted in an additive effect in reducing the emergence of adults.

4. The combined application of two biopesticides (M. brunneum or Spinosad) and Minx capsules was tested against the fungus gnats species on three different host plants (basil, parsley and poinsettia), under semi-greenhouse conditions. 


\section{Summary}

a. Fungus gnat females laid more eggs on parsley and poinsettia plants soils treated with Spinosad alone or in combination with Minx capsules as compared to the other treatments. Using basil plants, females preferred to lay more eggs in treatments with Minx capsules or the control in comparison to other treatments.

b. In all of three host plant species tested, Spinosad treatments alone or in combination with Minx significantly reduced the number of emerging adults.

The results compiled so far still require further improvements to make these co-formulations (e.g. biopesticides $M$. brunneum or Spinosad in combination with the botanical capsules) practically feasible for greenhouse producers to manage fungus gnat populations. 


\section{General introduction}

Dark-winged fungus gnats of the genus Bradysia spp. (Diptera: Sciaridae) are major insect pests of greenhouses and nurseries (Hamlen \& Mead, 1979). This genus comprises various species with the most important species B. impatiens Johannsen and B. coprophila Comstock (Wilkinson \& Daugherty, 1970, Harris et al., 1996). Additional species are found in greenhouse production systems, e.g. B. odoriphaga, B. agrestis and B. ocellaris (Cloyd, 2008). The larval stages of fungus gnats feed on fungi and other microorganisms in soils or decaying plant material (Kennedy, 1974, Cloyd, 2014). In addition, fungus gnat larvae cause a considerable damage in plants by feeding on healthy plant roots and tunneling into stems, disrupting water and nutrient uptake which results in wilting and stunted growth of plants (Wilkinson \& Daugherty, 1970a, Fawzi \& Kelly, 1982). Furthermore, fungus gnat larvae are capable to disseminate the soil-borne plant pathogens including Pythium spp., Fusarium spp., and Verticillium spp., from infected to non-infected plants (Gardiner et al., 1990, Gillespie \& Menzies, 1993, Stanghellini \& El-Hamalawi, 2005, Cloyd, 2015).

Fungus gnat adults only cause minimal plant damage; however, they are able to carry the aerial conidia of some fungal spores e.g. Botrytis cinerea, on their bodies, from infected to healthy plants (James at al., 1995, Cloyd, 2000). In general, adults reside around or near the surface of the substrate and females lay 100 to 200 eggs into crevices of the substrate (Cloyd, 2008). The life cycle of fungus gnat includes the egg, four larval instars, pupa and adult. The development time from eggs to adults takes between 20-28 days. The variation of the developmental time is dependent on diet quality, genetic variation and temperature (Wilkinson \& Daugherty, 1970a, Harris et al., 1996, Braun, 2011). Eggs hatch into larvae, which are found the top of the growing medium or plant tissues (Dennis, 1978). Besides the larval diets on decaying plant materials and healthy plants, it was observed that the larvae of Sciarids may be cannibalistic under certain conditions (Steffan, 1966, Wilkinson \& Daugherty, 1970a).

Growing medium containing high moisture contents (Hungerford, 1916) and various bacteria and fungi as food source are preferred for larval fungus gnats (Brues, 1946). The type of growing medium and high level of microbial activity could affect the abundance and the development of fungus gnat larvae (Anas \& Reeleder, 1988, Jagdale et al., 2004). For instance, Kennedy (1974) reported a rapid development and high survivorship of $B$. impatiens larvae when exposed to fungal diets e.g. Alternaria tenuis Nees or brewer's yeast (Saccharomyces cerevisiae Meyen ex E.C. Hansen). However, not all fungi are a suitable food source for fungus gnat larvae. For instance, 
larvae that fed on Trichoderma harzianum Rifai were not able to survive and complete their life cycle to adults (Cloyd et al., 2007).

Fungus gnats can be controlled by physical, chemical and biological approaches. Physical techniques such as moisture management, sanitation and scouting adults by yellow sticky cards are important to deal with fungus gnats in greenhouse production systems (Cloyd, 2015). The use of physical barriers such as sand and diatomaceous earth on the surface of the substrate helps to prevent female fungus gnats to lay eggs, although this strategy was not found to be satisfying in all cases (Cloyd, 2015). Raudenbush et al. (2014) used Growstones ${ }^{\mathrm{TM}}$ as a physical barrier to reduce fungus gnat adult emergence. Chemical control methods including synthetic insecticides and insect growth regulators (IGR) are commonly used to alleviate problems with fungus gnats in greenhouses (Lindquist et al., 1985, Cloyd, 2008). Ludwig \& Oetting (2001) reported that a wide range of IGRs (e.g. fenoxycarb and pyriproxyfen) could provide the highest reduction of fungus gnat emergence. However, the extensive use of chemical insecticides by greenhouse producers resulted in the development of resistance and also has harmful effects on non-target organisms, human and environment (Bartlett \& Keil, 1997, Cloyd, 2008). Hence, there is a need to develop strategies using biological control agents (BCAs) as an alternative management sterategy to control fungus gnats. BCAs offer a wide variety of agents, which have been shown to be effective against fungus gnats (Cloyd, 2015). BCAs such as predatory mites (e.g. Gaeolaelaps aculeifer, Hypoaspis miles), a rove beetle (Dalotia coriaria Kraatz) and biopesticides have been used to manage fungus gnat populations (Gillespie \& Quiring, 1990, Chambers et al., 1993, Harris et al., 1995, Carney et al., 2002). Entomopathogenic nematodes (EPN) as a biopesticide, specifically Steinernema feltiae, were found to be highly effective against fungus gnats (Harris et al., 1995, Jagdale et al., 2004).

In general, a common character of biological insecticides, principally derived from bacteria and fungi, is the production of metabolic compounds that could be toxic against insect pests (Quesada-Moraga et al., 2006). One of the microbial insecticides, Bacillus thuringiensis Berliner subsp. israelensis (Bt) was firstly reported by Osborne et al. (1985) for fungus gnats control. However, several studies reported low or no effect of Bt against fungus gnats (Cloyd \& Dickinson, 2006). One problem related to the application of Bt might be due to the short residual activity of this product in the substrate (Cloyd \& Dickinson, 2006). This bioinsecticide is characterized by producing crystalline inclusions containing proteins with a high insecticidal activity (Höfte \& Whiteley, 1989). Besides Bt, there have been some reports on neem-based products, exhibiting 
insecticidal properties against fungus gnats. Ludwig \& Oetting (2001) reported the reduction of $B$. coprophila adult emergence after exposure to neem with its active ingredient azadirachtin. This natural insecticide was shown to be effective in suppressing fungus gnat populations (Cloyd, 2008, Koller, 2011). This bioinsecticide has been reported to inhibit the release of ecdysteroids resulting in an incomplete moulting of immature insect (Mordue \& Nisbet, 2000).

Another naturally derived biorational insecticide used in this study is Spinosad. This microbial insecticide derived from metabolites of the Actinobacteria Saccharopolyspora spinosa (Mertz \& Yao, 1990) was registered for use in 100 crops (Thompson et al., 2000). This natural insecticide has a low toxicity for mammals (Cleveland et al., 2001) and is comprised of two components, spinosyn $A$ and spinosyn $D$, which are responsible for the insecticidal properties (Thompson et al., 2000). It has been reported that this biopesticide is a neurotoxin and acts by depolarizing insect nerve system including acetylcholine and GAPA receptors, resulting in a disruption of insect neurons, paralysis and death (Salgado, 1998). The strong insecticidal activity of Spinosad has been shown for several insect pests, particularly Diptera e.g. fruit flies (Tephritidae) (Stark et al., 2004, Yee, 2018) and eye gnats Liohippelates collusor (Chloropidae) (Jiang \& Mulla, 2006). This insecticide, according to the label, can be applied for controlling pest species e.g. thrips on a number of ornamental plant species including poinsettia, hibiscus, Chrysanthemum and transvaal daisy (Cloyd, 2009). The application of Spinosad against thrips in these plant species might also aid in fungus gnats management in greenhouse production system. Moreover, some studies have reported the attraction and oviposition of insect pests to Spinosad. Perez et al. (2007) reported the attraction of females Aedes aegypti (Diptera: Culicidae) to Spinosad. Scott (1998) also indicated the protrusion of the ovipositor of houseflies and expelling the eggs when exposed to Spinosad.

Entomopathogenic fungi (EPF) as microbial control agents gained increasing attention in recent years by researchers and enterprises. EPF are widely used as a part of integrated pest management (IPM) with low-hazard effects on human health and environment. Butt et al. (2001) stated that the most EPFs belong to the order of the Hypocreales. The commercially fungal products in this order are Beauveria bassiana (Balsamo) Vuillemin and Metarhizium anisopliae (Metschnikoff) Sorokin (Ascomycota: Hypocreales), which have been shown to be highly effective against a wide range of insects including dipteran insects. EPFs might be considered as contact bioinsecticides because of the fungal invasion by penetrating hyphae through the cuticle insects and releasing blastospores into the haemocoel (Pedrini, 2018). However, the death of the insect 
can be due to a number of factors, including a combination of mechanical damage, toxicosis and nutrients exhaustion (Hajek \& St. Lager, 1994). Additionally, EPFs are able to produce toxic substances known as secondary metabolites (Vey et al., 2001), resulting in immunosuppressive insects facilitating the fungal invasion (Roberts et al., 1992, Trienens \& Rohlfs, 2012). The main advantages of EPFs include the mass production of propagules, high efficacy to infect insects and the reasonable costs (Vega et al., 2008, Jackson et al., 2010).

The use of EPF needs to be evaluated as an alternative management strategy for controlling the fungus gnat species $B$. impatiens because information on the efficacy of EPFs against fungus gnats is limited so far. The effectiveness of B. bassiana for controlling the fungus gnat Lycoriella ingenua Dufour (Diptera: Sciaridae) in mushroom production was reported by Andreadis et al. (2016). Filotas et al. (2005) found that the shore flies Scatella stagnalis Fallen, known as a vector of fungal pathogens in greenhouses, is infected by B. bassiana (Stanghellini \& El-Hamalawi, 2005). Another entomopathogenic fungus, $M$. anisopliae has been shown to be effective in reducing fungus gnat larvae of L. auripilla Winnertz (Diptera: Sciaridae) in mushroom production (Tavoosi Ajvad et al., 2019).

A combined application of different, but compatible products has been recently suggested to improve the control efficacy in insect pests, simultaneously decreasing the amount of application and minimizing pest resistance and environmental concerns (Quintela \& McCoy, 1998, Lacey et al., 2001). Tavoosi Ajvad et al. (2019) reported the combined application of M. anisopliae and a predatory mite Gaeolaelaps aculeifer to increase the control efficiency of L. auripilla in mushroom production. The studies of Acharya et al. (2019) also indicated the enhanced control efficiency of the fungus gnat $B$. impatiens by using a combination of an entomopathogenic nematode (EPN) Heterorhabditis indica and the predatory mite Stratiolaelaps scimitus. Additionally, the combined application of EPNs (e. g. Steinernema feltiae or Heterorhabditis megidis) and IGR cyromazine caused a higher mortality in L. solani (Diptera: Sciaridae) larvae in mushroom production systems (Sznyk-Basalyga \& Bednarek, 2003).

A potential approach to enhance the control efficiency of the fungus gnat species $B$. impatiens might be an "attract and kill" strategy. By using this mechanism, according to the study of El-Sayed et al. (2009), an insect is attracted towards an attractive source with a specific cue including a semiochemical and is thus more exposed to the killing agents (e.g. spore suspensions). This strategy might lead to a behavioral manipulation of the insect, which contributes to the higher control efficacy (Vernon et al., 2015). Therefore, a combined application of biological 
General introduction

products might be an alternative for controlling $B$. impatiens and needs studies under both laboratory and greenhouse conditions. 


\section{Objectives}

\section{Objectives}

The present study aimed at developing a new strategy using a combined application of a biopesticide and a botanical compound, formulated in a slow release capsule containing mint oil, to control fungus gnat Bradysia impatiens. Fungus gnat control was addressed with an "attract and kill" strategy in laboratory experiments by combining a biopesticide (e.g. Metarhizium brunneum or Spinosad) as a killing agent and a capsule containing a botanical compound (Minx) as an attractive agent. Additionally, fungus gnat control was addressed in greenhouse experiments by combining biopesticides (e.g. Metarhizium brunneum or Spinosad) and the Minx capsules using three host plants species (basil, parsley and poinsettia).

Specifically we

1. Tested the response of fungus gnat larvae when exposed to pure fungal cultures or to substrates treated with the fungal cultures (Chapter 1 )

a. Are fungus gnat larvae able to feed on pure fungal cultures such as Metarhizium brunneum, Beauveria bassiana and Acremonium strictum?

b. Are larval stages of fungus gnat B. impatiens affected entomopathogenic fungi (EPF) when applied as pure fungal diets or mixed within the substrate?

2. Evaluated a combined application of a biopesticide and a botanical compound against fungus gnat larvae (Chapter 2)

a. Do botanical compounds in two different formulations have repellent or attractant properties for fungus gnat larvae?

b. Does a combined application of a biopesticide (M. brunneum or Bacillus thuringiensis, Bt) and a botanical compound enhance the control efficiency of fungus gnats in an "attract and kill" strategy?

3. Evaluation of a combined application of a biopesticide and a botanical compound on larval fungus gnats and oviposition of fungus gnat females (Chapter 3)

a. Do biopesticides ( $M$. brunneum, Spinosad and Neem Azal) in combination with a botanical compound build up a better control in an "attract and kill" strategy targeting fungus gnat larvae?

b. Do combined applications of these biopesticides with a botanical compound affect on attraction and oviposition of fungus gnat female? 


\section{Objectives}

4. Evaluated a combined formulation of a biopesticide and a botanical compound against fungus gnat using three host plants species (basil, parsley and poinsettia) under greenhouse conditions (Chapter 4)

a. Do combined formulations of biopesticides (M. brunneum or Spinosad) with a botanical compound influence the oviposition behavior of fungus gnats in soils of host plants species?

b. Can these combined formulations reduce sufficiently the adult emergence of fungus gnat in these host plant growing systems? 
Chapter 1

Utilization of entomopathogenic fungi, Metarhizium brunneum and Beauveria bassiana to control larvae of fungus gnats

Mahsa Dehghani* and Stefan Vidal

* Corresponding author 


\title{
Utilization of entomopathogenic fungi, Metarhizium brunneum and Beauveria bassiana to control larvae of fungus gnats
}

M. Dehghani*and S. Vidal

Georg-August-Universität Göttingen, Department of Crop Sciences, Agricultural Entomology, Grisebachstrasse 6, 37077 Göttingen, Germany

*Corresponding author: E-mail address: mdehgha@gwdg.de

\begin{abstract}
Fungus gnats, including the common species Bradysia impatiens Johannsen (Diptera: Sciaridae) are considered serious pests in greenhouses. Entomopathogenic fungi as biological control agent are being developed in controlling pest in IPM strategy. Therefore, a series of laboratory experiments were conducted to investigate the potential of entomopathogenic fungi (two isolates of Metarhizium brunneum, three isolates of Beauveria bassiana and Acremonium strictum) to control larvae of fungus gnats (B. impatiens). In first series of bioassays, larvae that had fed on the pure fungal cultures allowed to complete the life cycles to adults. Survival of larvae and emergence rate of adults reduced after exposure larvae to different fungal strains; however, isolates of $B$ bassiana and $A$. strictum exhibited a high survival of fungus gnat larvae. Entomopathogenic fungi, $M$. brunneum was more effective in reducing survival of larvae, with the emergence rate of adults ranging from $3.5 \%$ ( $M$. brunneum ENTO-B-1) to $4.5 \%$ ( $M$. brunneum $\mathrm{Cb} 15)$. In subsequent bioassays, one selected isolates of $M$. brunneum ( $\mathrm{Cb} 15)$, one isolate of $B$. bassiana (EABb04/01Tip) and A. strictum were applied at a rate of $1 \times 10^{7}$ spores $/ \mathrm{ml}$ into each cup filled with growing medium. 24 hours post treatment, twenty newly third instar larvae were transferred into each experimental cup, and adult emergence monitored during two weeks using yellow sticky cards. This experiment was conducted in two different substrates containing different components. Data collected from these trials demonstrated that the larvae were more susceptible to infection by $M$. brunneum strain $\mathrm{Cb} 15$ as compared to the other fungal strains, resulted in less number of adults emerged from this fungal strain varying from $21 \%$ to $25 \%$ in both substrates. These findings suggest that entomopathogenic fungal strain, M. brunneum may have a potential to be used for management of fungus gnats.
\end{abstract}

Keywords: fungus gnats, entomopathogenic fungi, fungal infection, biological control, adult emergence 


\section{Introduction}

Fungus gnats in the genus Bradysia spp. (Diptera: Sciaridae) are major insect pests of greenhouses production systems. Within the genus Bradysia spp., B. impatiens Johannsen and B. coprophila Comstock are the most commonly encountered species in greenhouses and nurseries (Wilkinson \& Daugherty, 1970a, Lindquist et al., 1985). Other species are reported as crop pests include $B$. odoriphaga, B. agrestis and B. ocellaris (Cloyd, 2008). Whereas fungus gnat larvae are the causal agents of the damage on crops, adults are causing minimal plant damage (Cloyd, 2000). The study of Kennedy (1976) indicated Sciarid adults as generally aphagous, although they have been reported to consume liquids such as nectar (Mercier, 1911), molasses (Hungerford, 1916) and organic ooze (Steffan, 1966). It has been shown that fungus gnat adults can transmit the aerial conidia of some soil-borne plant pathogenic fungi, on their bodies, such as Botrytis cinerea, Fusarium avenaceum and Verticillium albo-atrum from diseased to healthy plants (Gillespie \& Menzie, 1993, James et al., 1995, El-Hamalawi, 2008). In greenhouses, fungus gnat larvae feed mainly on decaying plant material and fungi (Kennedy, 1974). They can cause direct plant damage by feeding on the roots and stems, limiting the ability of water uptake and nutrients by the plants, ultimately resulting in wilting and stunted growth (Wilkinson \& Daugherty, 1970a). In addition, feeding damage caused by larvae may also promote plant infection by pathogenic fungi (Leath \& Newton, 1969, Graham \& McNeill, 1972). Larvae are capable of transmitting the fungal diseases including Pythium spp., Fusarium spp., and Verticilium spp. from infected to healthy plants (Gardiner et al., 1990, Gillespie \& Menzie, 1993). Some studies have showed that the oospores of Pythium spp. can survive after passage through the digestive tract of $B$. impatiens larvae, which might be transmitted into the healthy plants via the feeding process (Gardiner, 1990, Cloyd, 2015). Kennedy (1974) determined that fungus gnat larvae are able to complete their development more rapidly and enhance the survival rate when exposed to fungal diets of Alternaria tenuis Nees or brewer's yeast (Saccharomyces cerevisiae Meyen ex E.C. Hansen) as compared to the non-fungal diets. Hence, the type of food source fed by fungus gnat larvae influence survival rates, emergence rate of adults and reproductive potential of females (Cloyd, 2008).

Several control strategies, such as the application of synthetic and biorational insecticides exist for the control of fungus gnats. Insecticides are mainly used to deal with fungus gnats as drench to the growing medium (Hamlen \& Mead, 1979, Lindquist et al., 1985). However, the extensive use of synthetic insecticides by greenhouse producers might cause undesirable effects, such as insecticide resistance and environmental concerns (Bartlett \& Keil, 1997). Alternative management strategies, including biological control agents (BCAs) are being currently used and 
developed successfully for managing fungus gnat population (Cloyd, 2015). These biological agents, such as predatory mites (Gillespie \& Quiring, 1990), entomopathogenic nematode (EPN) (Dreistadt, 2001, Jagdale et al., 2004, Acharya et al., 2019), natural insecticides Bacillus thuringiensis (Bt) (Osborne at al., 1985, Cloyd \& Dickinson, 2006) and Neem (Cloyd, 2008) have been shown to be marginally effective against fungus gnats in greenhouses and nurseries. Therefore there is a need for the development of the alternative strategies to establish an efficient management. Entomopathogenic fungi (EPF) could provide an effective complementary system in an integrated pest management (IPM) strategy. Entomopathogenic fungi were widely used as a part of IPM strategies for pest management. Among them, Metarhizium anisopliae (Metschnikoff) Sorokin and Beauveria bassiana (Balsamo) Vuillemin are known as biological control agents in controlling dipteran insects (Watson et al., 1995).

The use of EPF needs to be evaluated as a potential approach in plant protection strategies to enhance control efficacy of fungus gnat Bradysia spp. populations. Andreadis et al. (2016) reported the susceptibility of fungus gnats Lycoriella ingenua Dafour (Diptera: Sciaridae) adults to infection by $B$. bassiana while this strain was ineffective on larval development of $L$. ingenua. The infection of shore fly, Scatella tenuicosta Collin (Diptera: Ephydridae) adults, an important vector of several plant pathogenic fungi, by B. bassiana has been reported by Filotas et al. (2005). A study by Stanghellini \& El-Hamalawi (2005) also indicated B. bassiana as a biopesticide for the control of this shore fly. Additionally, M. anisopliae has been shown to be highly effective to control fungus gnat L. auripilla (Tavoosi Ajvad et al., 2019).

Studies on the activity of fungal microorganisms could contribute to the discovery of fungal endophytes, such as Acremonium strictum, which is known as a mediator of plant-herbivorous insect interactions. This endophytic fungus (EF) has been described as a non-specialized and widespread soil born fungal endophyte indirectly influencing behavioral changes of herbivores insects (Jallow et al., 2004). Hence, the performance and preference of herbivorous insect might be influenced by endophytic fungi inoculated in host plants. For instance, Jaber \& Vidal (2010) demonstrated that behavioral modification of Helicoverpa armigera larvae (Hübner) (Lepidoptera: Noctuidae) on bean plants inoculated with A. strictum. Most studies have investigated the role of this fungus as a mediator of plant-insect interactions. The objective of this study was to evaluate the potential of $A$. strictum as a biocontrol agent against fungus gnat larvae.

The objectives of this study were: (1) to evaluate whether fungus gnat larvae are able to feed on fungal mycelium or spores (isolates of two Metarhizium brunneum, three Beauveria bassiana and one Acremonium strictum) and (2) to determine whether the larval stage of $B$. 
impatiens are affected by these fungal cultures when applied as pure fungal diets or mixed within the substrate.

\section{Materials and methods}

Insect rearing

The fungus gnat species B. impatiens, used in this study, was obtained from a colony maintained in Agricultural Entomology Section, Crop Science Department, Georg-August Universität Göttingen, Germany. Adults were reared on a favorable substrate (350 g) containing $50 \% \mathrm{v} / \mathrm{v}$ medium milled white peat, $30 \% \mathrm{v} / \mathrm{v}$ frozen-through black peat, $20 \% \mathrm{v} / \mathrm{v}$ green waste compost, $2 \mathrm{~kg}$ lime $/ \mathrm{m}^{3}, 1$ $\mathrm{kg}$ Radigen $/ \mathrm{m}^{3}$ and $6 \mathrm{~kg}$ horn shives / $\mathrm{m}^{3}$ (Klasmann-Deilmann GmbH; Geeste, Germany) supplemented with oatmeal (3 gr) and shredded potato (Cloyd \& Zaborski, 2004). Fungus gnat's rearing was maintained at $23 \pm 2^{\circ} \mathrm{C}$ with a 16 -h photoperiod. To obtain even-aged third-instar fungus gnat larvae, adults from the permanent colonies were allowed to oviposit in a small container containing moist growing medium as described above for rearing adults (KlasmannDeilmann $\mathrm{GmbH}$; Geeste, Germany) supplemented with oatmeal and potato. 48h post oviposition, this container was placed in a room at $23 \pm 2^{\circ} \mathrm{C}$ to allow development to third instar larvae. The newly hatched third instar larvae were collected from the surface of the growing medium via placing a cooked potato disk on top of the medium (Cabrera et al., 2003). The larvae were removed from the potato disks using a floating technique and used in the bioassays.

\section{Fungal isolates}

Five fungal isolates were used in the experiments, including three strains of $B$. bassiana (EABb04/01-Tip, Bb 1022 and Bv061), two strains of M. brunneum (Mb Cb 15 and ENTO-B-1) and A. strictum as detailed in Tab. 1. The fungal strains used for this study are maintained at the Agricultural Entomology Section. These fungal cultures were grown on potato extract glucose agar (PDA) (Carl Roth GmbH \& Co. KG, Karlsruhe, Germany) and incubated at $25 \pm 1^{\circ} \mathrm{C}$ in a dark climatic chamber (Biologischer Klimaschrank WB 750, Mytron Bio- und Solartechnik GmbH, Heilbad Heiligenstadt, Germany). The cultures were allowed to grow for ca. 3 weeks, after which fresh spores were harvested by scraping the surface of medium plate into a $500-\mathrm{ml}$ glass beaker containing $50 \mathrm{ml}$ sterile demineralized water plus Tween 80 (0.1\% v/v; Carl Roth ${ }^{\circledR}$, Germany). The conidial suspension was then homogenized by vortexing, and filtered through a sterilized fine mesh to remove mycelium and agar residues. The spore suspension was verified with light microscopy (200X magnification, BH2-HLSH, Olympus Corporation, Tokyo, Japan) and adjusted to 
the desired concentration of $1 \times 10^{7}$ spores/ml using a Neubauer Hemocytometer (0.1 mm depth) (Thoma CE, Paul Marienfeld GmbH \& Co.KG, Lauda-Königshofen, Germany).

Table 1: Fungal isolates and origins used in this study.

\begin{tabular}{|c|c|c|c|}
\hline Fungal Species & Isolate & Insect Host & Site of origin \\
\hline Beauveria bassiana & EABb04/01-Tip & Timaspis papaveris (Kieffer) & Spain \\
\hline B. bassiana & Bv 061 & Bemisia tabaci (Gennadius) & Colombia \\
\hline B. bassiana & Bb 1022 & Rhyacionia buliana (Schiff.) & Canada \\
\hline Metarhizium brunneum & $\mathrm{Cb} 15$ & Agriotes spp. & Austria \\
\hline M. brunneum & ENTO-B-1 & Agriotes spp. & Germany \\
\hline Acremonium strictum & \multicolumn{3}{|c|}{ (DSMZ-GmbH Braunschweig) } \\
\hline
\end{tabular}

\section{Experimental set-up}

Larval survival on pure fungal cultures

25 third instar larvae that had been placed on water agar (Agar-Agar, Kobe I, Carl Roth GmbH \& Co. KG, Karlsruhe, Germany) plates for $6 \mathrm{~h}$ to clear their guts were transferred to each of the fungal cultures using a fine brush (Table 1) and allowed to complete their life cycle to adult flies. For the control treatments, the fungal culture plates were replaced with PDA without any fungal culture. Plates were incubated at $25 \pm 1^{\circ} \mathrm{C}$ and $65 \% \mathrm{RH}$ in a climatic chamber (Biologischer Klimaschrank WB 750, Mytron Bio- und Solartechnik GmbH, Heilbad Heiligenstadt, Germany). The number of emerged adults was monitored daily. A total of eight replicates for each treatment were set up, each containing 25 larvae, totally 200 individuals per treatment. The wing length of emerged fungus gnat adults from two fungal strains (Beauveria bassiana EABb04/01-Tip and $A$. strictum), which yielded the higher numbers of emerged adults, was measured under a binocular microscope (ZEISS, Carl Zeiss Microscopy GmbH, Jena, Germany). Additionally, a group of 15 larvae were allowed to feed on M. brunneum and B. bassiana for $48 \mathrm{~h}$. These larvae were then surfacesterilized with sodium hypochlorite (0.5\%) (Carl Roth GmbH \& Co. KG, Karlsruhe, Germany) for 30s and rinsed two times in sterile distilled water (SDW) (as described by El-Hamalawi, 2008). The surface-sterilized larvae were anesthetized with a gentle stream of $\mathrm{CO}_{2}$ and then placed on filter paper (90 mm diameter, Schleicher \& Schuell GmbH, Dassel, Germany). Afterwards, the guts of larvae that had fed on these fungal cultures were individually removed, dissected under a binocular microscope (ZEISS, Carl Zeiss Microscopy GmbH, Jena, Germany) and placed on semiselective medium (SDA: Tryptone/peptone $5 \mathrm{~g}+$ Glucose Monohydrat $10 \mathrm{~g}+$ Agar-Agar $9 \mathrm{~g} / 500$ 
ml water) (Carl Roth $\mathrm{GmbH} \&$ Co. KG, Karlsruhe, Germany) supplemented with the antibiotics cycloheximide $25 \mathrm{mg} / 500 \mathrm{ml} \mathrm{H} \mathrm{H}_{2}$ (Carl Roth GmbH \& Co. KG, Karlsruhe, Germany), streptomycin $50 \mathrm{mg} / 500 \mathrm{ml} \mathrm{H} \mathrm{H}_{2}$ (Carl Roth GmbH \& Co. KG, Karlsruhe, Germany), tetracycline $25 \mathrm{mg} / 500 \mathrm{ml}$ $\mathrm{H}_{2} \mathrm{O}$ (Sigma-Aldrich, Steinheim, Germany) and the fungicide dodine $122 \mu \mathrm{l} / 500 \mathrm{ml} \mathrm{H}_{2} \mathrm{O}$ (Fungicida Syllit FLO, Bayer Crop Science) (Strasser et al., 1996). This experiment was carried out in a climate chamber at $25 \pm 1^{\circ} \mathrm{C}$ and $65 \pm 5 \% \mathrm{RH}$. Three weeks after incubation, fungal growth was evaluated under a microscope to check whether fungal mycelium or spores have been ingested.

\section{Effectiveness of entomopathogenic fungi to control fungus gnat larvae}

The screening experiments included the evaluation of the efficacy of three fungal strains, $M$. brunneum (Cb 15), B. bassiana (EABb04/01-Tip) and A. strictum, in impacting the development of larvae. In the first trial (experiment 1), plastic cups (300 ml, Nette Papier $\mathrm{GmbH}$, Göttingen, Germany) were filled with $250 \mathrm{ml}$ standard substrate as detailed in Tab. 2 (Klasmann-Deilmann GmbH Geeste, Germany) supplemented with bio fertilizer (Phytoperls: 8 gr per liter substrate; Klasmann-Deilmann GmbH Geeste, Germany) and green waste compost "TerrAktiv" (0.3 L per liter substrate, Klasmann-Deilmann GmbH Geeste, Germany). $10 \mathrm{ml}$ of the fungal suspensions were applied at a rate of $1 \times 10^{7}$ spore $/ \mathrm{ml}$ directly to the substrate and were homogenously incorporated. Control cups were treated with $10 \mathrm{ml}$ sterile demineralized water. $24 \mathrm{~h}$ after the treatment, 20 newly moulted third instar fungus gnat larvae were transferred into each treated or control cup using a size 3/0 paint brush (Davinci, Germany) and allowed to complete their life cycle. These cups were then incubated in a growth chamber at $25 \pm 1^{\circ} \mathrm{C}$ and $65 \pm 5 \% \mathrm{RH}$ with a photoperiod: 16:8 h (Biologischer Klimaschrank WB 750, Mytron Bio- und Solartechnik GmbH, Heilbad Heiligenstadt, Germany) and each cup was closed with a lid. At the top of each cup a yellow sticky trap $(2.5 \times 2.5 \mathrm{~cm})$ was attached to monitor the number of emerging adults. A total of 10 replications for each treatment were set up and observed over a period of two weeks. The trial was terminated when no new adults were counted within a period of 4 days. 
Table 2: Compounds of standard substrate (Substrate 1) used in this study.

\begin{tabular}{|c|c|}
\hline Material & Quantity \\
\hline Humintorf hell Deutschland & $0.700 \mathrm{~m}^{3}$ \\
\hline WT Frästorf Baltikum fein & $0.300 \mathrm{~m}^{3}$ \\
\hline Kalksteinmehl -fein- mit 5\% MgO, lose & $7.500 \mathrm{~kg}$ \\
\hline NPK-Dünger 12/14/24 -Profi- & $1.200 \mathrm{~kg}$ \\
\hline Netzmittel/Wetting Agent Struktur fein & $0.050 \mathrm{~kg}$ \\
\hline $\begin{array}{l}\text { pH 5,2 Gew.max } 330 / \mathrm{kg} / \mathrm{cbm} \\
\mu \mathrm{S} 450 \text { mit minimale Absiebung (400 Umdrehungen) }\end{array}$ & \\
\hline
\end{tabular}

In a second trial (experiment 2), the set up and procedure was the same as described in the $1^{\text {st }}$ trial. In this experiment, plastic cups were filled with a $250 \mathrm{ml}$ substrate mixtures of peat soil (Fruhstorfer Erde Typ P 25; Hawita Gruppe GmbH, Vechta, Germany), supplemented with $10 \mathrm{ml}$ fertilizer NPK ((15-10-15(+2); Hakaphos ${ }^{\circledR}$ Blau). The substrate received $10 \mathrm{ml}$ conidial suspension of fungal cultures, using the isolates M. brunneum ( $\mathrm{Cb}$ 15), B. bassiana (EABb04/01-Tip) and $A$. strictum $\left(1 \times 10^{7}\right.$ spore/ml). Control substrates were treated with sterile demineralized water with the same volume as the fungal cultures. $24 \mathrm{~h}$ post treatment, 20 newly moulted third instar larvae were transferred into each cup and were incubated in a climate chamber (photoperiod: 16:8 h; air temperature and humidity: $\left.25 \pm 1^{\circ} \mathrm{C}, 65 \pm 5 \%\right)$. The total number of adults emerging from each treatment was evaluated after 14 days incubation.

\section{Data analysis}

All statistical analyses were computed with $\mathrm{R}$ version 3.5.3. Survival of larvae following exposure to the pure fungal cultures were analyzed with Kaplan-Meier survival analysis with differences in survival rates of larvae using the Breslow test and the differences of survivorship between treatments using the Tukey test at $5 \%$ level of significance. In addition, the wing length of emerged adults from the two fungal cultures $B$. bassiana and $A$. strictum was analyzed with a one-way ANOVA and the differences between two fungal cultures were tested with two-sample t-test $(\alpha=$ 0.05). All data were first checked for normality and error variance of homoscedasticity. In the screening experiments, the mean total number of adults emerging from fungal cultures in the first trial (experiment 1) was analyzed using one-way ANOVA and in second trial (experiment 2) GLM 
with quasipoisson distribution was used. The differences between treatments were tested by the Tukey Honestly Significance difference test at a 5\% level of significance.

\section{Results}

Larval survival on pure fungal cultures

Survival rates of larvae progressively declined from first day after treatment onwards when exposed to the different fungal cultures (Chisq $=328.14$, $d f=6, p<0.001$; Fig. 1). The control group of larvae survived only for two days following exposure to pure PDA, significantly less long than compared to all other fungal treatments, followed by larvae reared on fungal cultures $M$. brunneum strains ENTO-B-1 and Cb 15 during 15 days post exposure. Larvae exposed to $M$. brunneum (ENTO-B-1 and Cb 15) were more susceptible, resulting in only $3.5 \%$ and $4.5 \%$ adult emergence (total $\mathrm{N}=200$ ) compared to the other treatments. Subsequently, the survival of larvae after exposure to B. bassiana strains Bb 1022 and Bv 061 indicated an intermediate survivorship; resulted in $7 \%$ and $8 \%$ larvae (total $N=200$ ) successfully completing their life cycle to adult flies, with no significant differences in emergence rate of adults between these treatments $(p=0.554)$. In contrast, the emergence of adults from the larvae treated with the fungal strains $A$. strictum and B. bassiana EABb04/01-Tip, although not significantly, exhibited a long survival during 17 days post infection, resulting in an overall emergence rate of adults $9 \%$ and $13.5 \%$ (Total $N=200$ ), respectively. Wing length of fungus gnat adults emerged from pure fungal culture $B$. bassiana EABb04/01-Tip (mean $\pm \mathrm{SE} ; 1.42 \pm 0.04$ ) and A. strictum (mean $\pm \mathrm{SE} ; 1.39 \pm 0.0 .06$ ) did not significantly differ $(t=0.33, d f=12, p=0.75$; Fig. 2 ). 


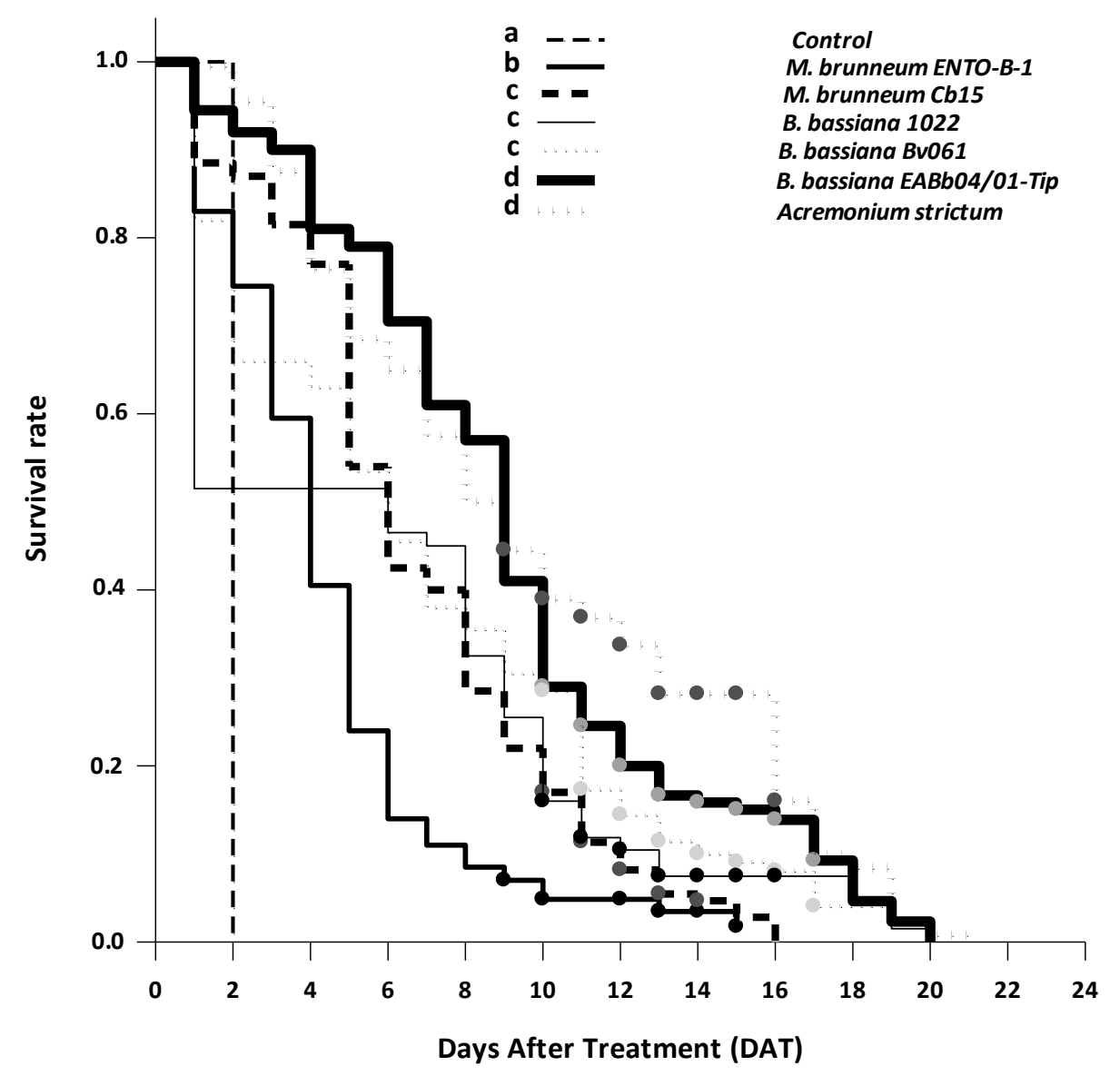

Fig. 1: Survival rate of fungus gnat larvae following direct exposure to the entomopathogenic fungal cultures or PDA (control) analyzed using Kaplan-Meier survival analysis. Data represents the mean survival ratio of larvae $( \pm \mathrm{SE}$ ). The survivorship of larvae between different treatments was analyzed according to the Tukey HSD test $(p<0.05)$. 


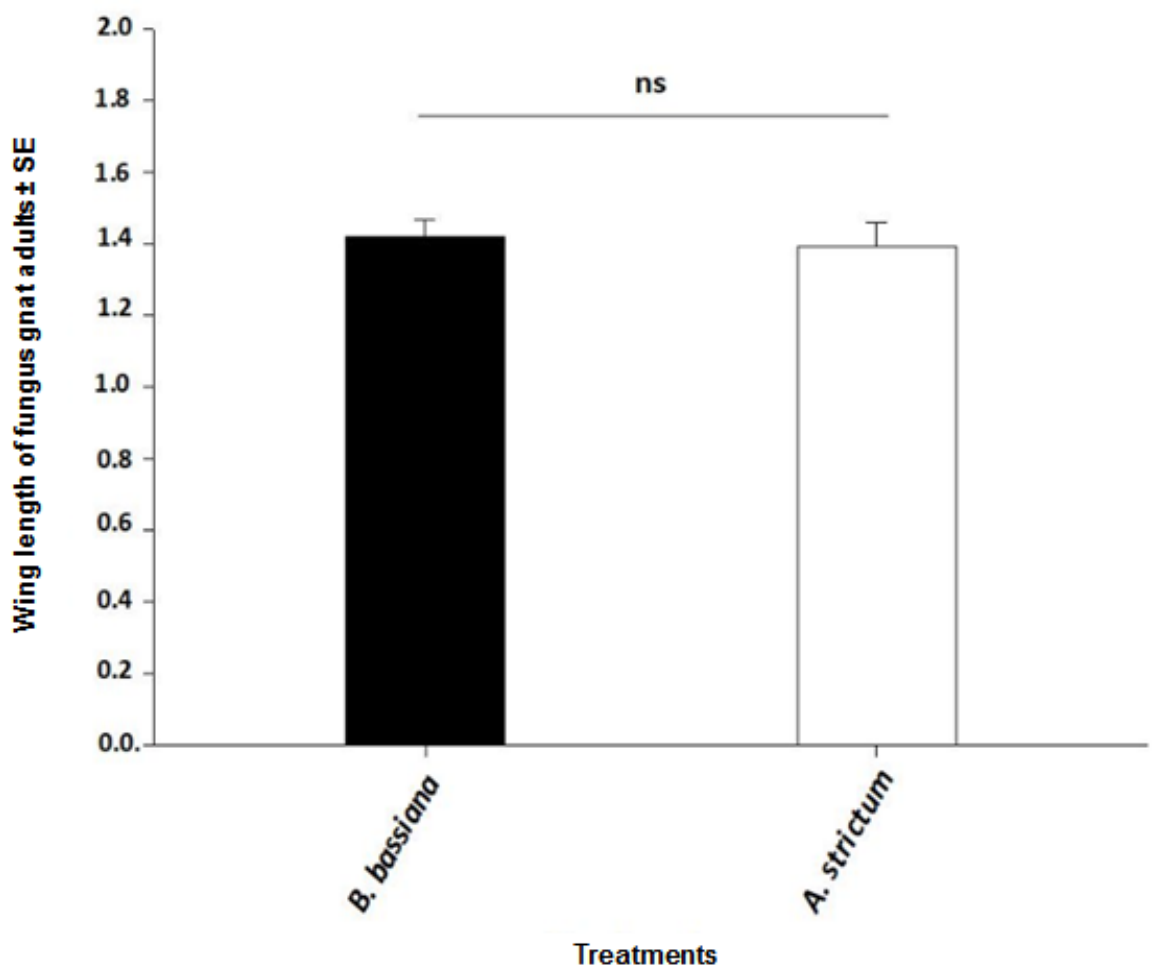

Fig. 2: The wing length of fungus gnat adults $(\mathrm{mm})$ (mean $\pm \mathrm{SE}$ ) hatched from two fungal cultures (B. bassiana EABb04/01-Tip and A. strictum). The wing length of adults between two treatments is not significantly different, according to two-sample $t$-test ( $t=0.33, \mathrm{df}=12, \mathrm{p}=0.75$ ); ns: not significant

\section{Effect of entomopathogenic fungi to control larvae of B. impatiens}

The three selected fungal strains ( $M$. brunneum $\mathrm{Cb}$ 15, B. bassiana EABb04/01-Tip and $A$. strictum; experiment 1) used in this experiment reduced the survival rate of fungus gnat larvae, fewer adults hatching from the treated substrate with pure fungal cultures as compared to the control $\left(F_{7,72}=5.64, p<0.001 ;\right.$ Fig. 3). Among fungal cultures, fungus gnat larvae were more susceptible to the treatment fungal culture $M$. brunneum $\mathrm{Cb} 15$, although not significantly, compared to the other fungal strains; this EPF significantly reduced the emergence of adults in substrate with fertilizer $(22.5 \%)$ and without fertilizer $(21 \%)$ as compared to the control treatments $\left(F_{3,36}=\right.$ $11.88, p<0.001$ ). Additionally, the number of fungus gnat adults from $A$. strictum in presence of fertilizer (23.5 \%) and absence of fertilizer (22\%), respectively, were significantly less than the number of adults emerged from the control treatments $\left(F_{3,36}=15.38, p<0.001\right)$. The number of adults emerging from $M$. brunneum $\mathrm{Cb} 15$ was slightly lower than in the fungal strain $A$. strictum, but this difference was not significant $\left(F_{3,36}=0.073, p=0.97\right)$. The fungal culture $B$. bassiana 
EABb04/01-Tip did not significantly reduce the survival rate of larvae; resulting in a higher emergence rate of adults ranging from $29.5 \%$ without fertilizer to $31.5 \%$ with fertilizer, respectively, with no significant difference between these treatments or the control $\left(F_{3,36}=3.49, p\right.$ $>0.01$ ). The data collected from this experiment also showed that the addition of the fertilizer did not result in significant differences within treatments in terms of the emergence of adults. However, the number of emerging adults from the substrate containing fertilizer was slightly, but not significantly, higher than the substrate without fertilizer $\left(F_{1,18}=0.05, p=0.82\right)$.

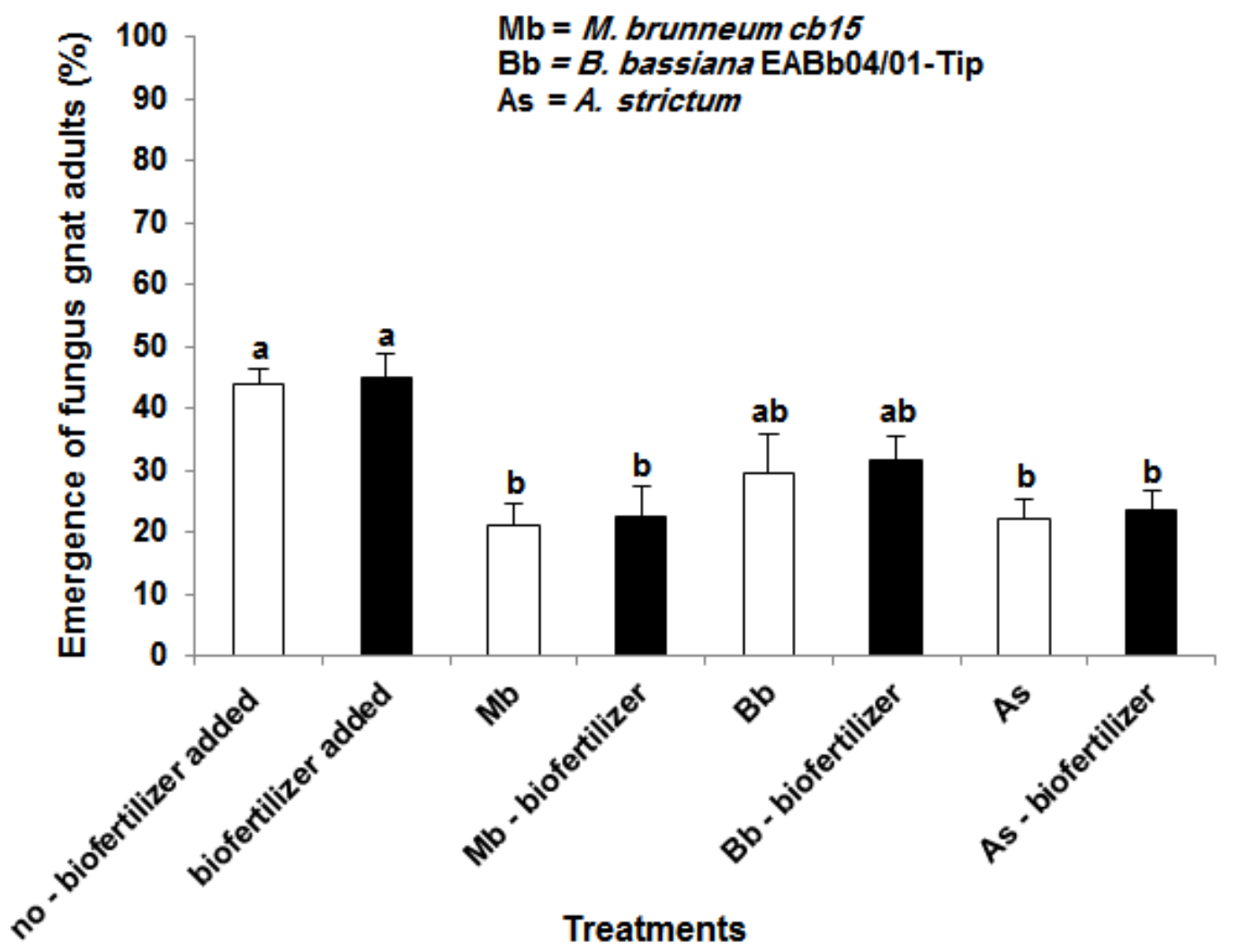

Fig. 3: Emergence of fungus gnat adults (\%) (Mean \pm SE) from larvae exposed to entomopathogenic fungi ( $M$. brunneum and B. bassiana) and A. strictum in a standard substrate (substrate 1) in presence and absence of biofertilizer. Means followed by a different letter are significantly different (Tukey HSD test) $\left(F_{7,72}=5.64, p<0.05\right)$.

In a second bioassay (experiment 2), the mean total number of emerged adults from substrates treated with the spore suspensions was also significantly lower than the emergence of adults from the control treatment $\left(F_{7,72}=8.78, p<0.001\right.$; Fig. 4). Among all fungal strains tested, two fungal strains, $A$. strictum and $M$. brunneum $\mathrm{Cb} 15$, has been shown to be highly effective against fungus gnat larvae, resulting in the emergence rate of adults ranging from $23.5 \%$ to $25 \%$, with no 
significant difference between these treatments $\left(F_{3,36}=0.05, p=0.98\right)$. Additionally, larval stage of fungus gnats were lower susceptible to infection by fungal strain B. bassiana (EABb04/01-Tip) in comparison to the other fungal strains $\left(F_{5,54}=2.78, p<0.05\right)$. This fungal culture could reduce the numbers of fungus gnat adults when compared to the control treatment with or without fertilizer NPK addition. Furthermore, the adult emergence of fungus gnats did not significantly differ between the substrates with and without fertilizer NPK $\left(F_{1,18}=0.09, p=0.75\right)$.

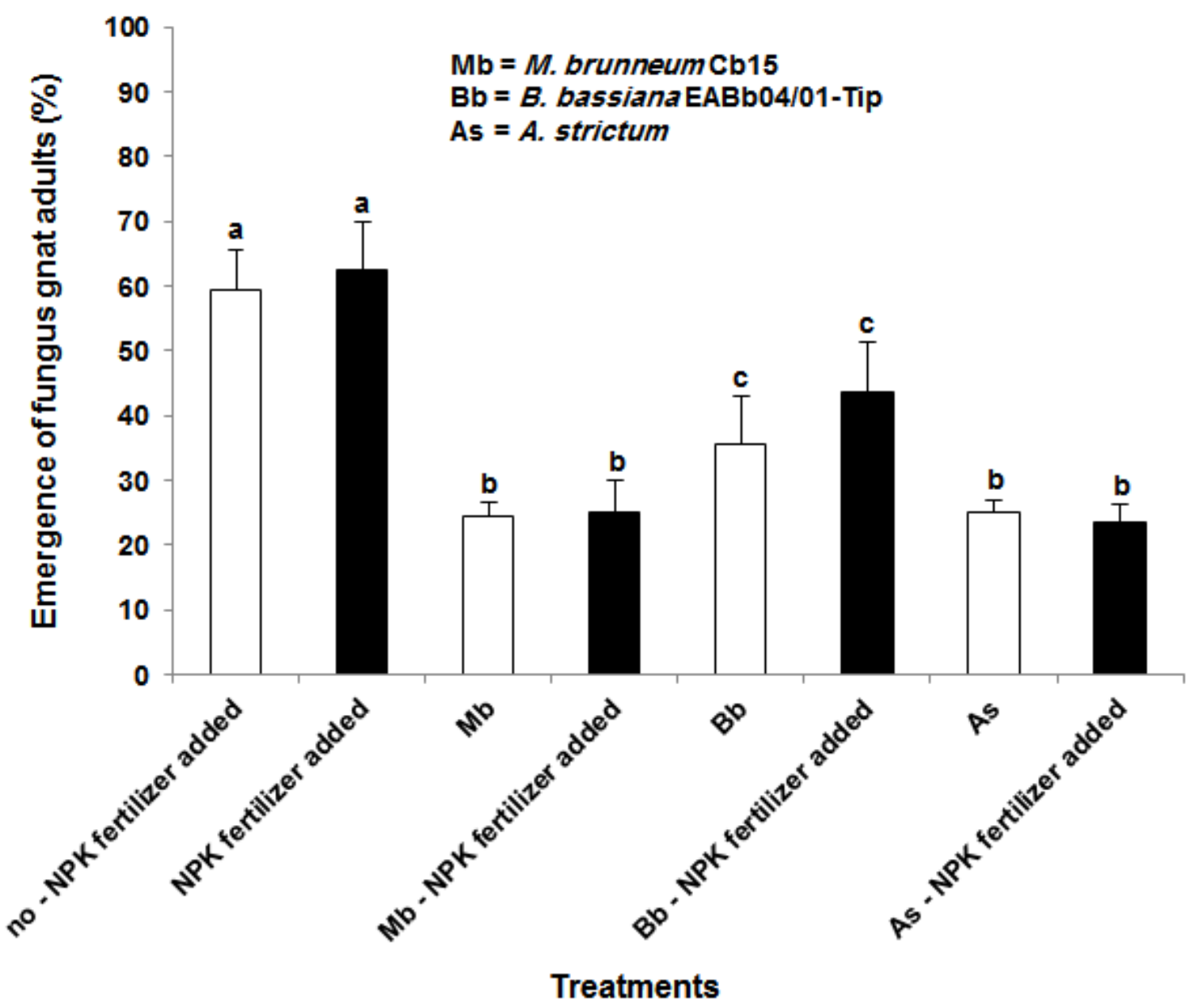

Fig. 4: Emergence of fungus gnat adults (\%) (Mean $\pm \mathrm{SE}$ ) after larval exposure to different fungal cultures (M. brunneum, B. bassiana and A. strictum) in a growing substrate (Fruhstorfer Erde Typ P) in presence and absence of NPK fertilizer. Treatments with the same letters above bars are not significantly different according to the Tukey HSD test $\left(F_{7,72}=8.78, p<0.05\right)$.

\section{Discussion}

This study demonstrated that $B$. impatiens larvae are able to survive even on pure fungal diets or in the substrate treated with EPF spore suspensions. The ingested spores of the fungal strains ( $M$. brunneum and $B$. bassiana) retained their viability and germination capability after passage 
through the gut of fungus gnat larvae. Hence, these results provide evidence that fungus gnat larvae are able to survive even on pure entomopathogenic diets. Some fungal isolates e.g. $B$. bassiana and $A$. strictum as pure fungal diets allowed a high percentage of survivorship. Larval stages of fungus gnats were more susceptible to $M$. brunneum in comparison to other fungal strains, which resulted in a reduced number of adults emerging. The larval stage of fungus gnats were only slightly affected by entomopathogenic fungi (EPF), when spore suspensions were mixed with the growing medium. Less larvae of $B$. impatiens survived after exposure to the spore suspensions of M. brunneum applied to the substrate. Tavoosi Ajvad et al. (2019) reported > $50 \%$ larval mortality of fungus gnats $L$. auripila after exposure to the fungal suspenisons of $M$. anisopliae $\left(10^{7}\right.$ and $10^{8}$ spores $\left./ \mathrm{ml}\right)$ applied to the compost of mushroom production. However, the study of Dobrindt (2013) indicated that the spore suspenisons of M. anisopliae did not significantly reduce the numbers of fungus gnat adults emerging. The variation in Metarhizium spp. efficacy for fungus gnat control might be related to the fungal strain, the application techniques and the time between fungus and larvae application and soil substrate type.

In addition, fungus gnat larvae could survive after feeding on pure fungal culture $B$. bassiana or in the substarte treated with this EPF. B. impatiens larvae were less susceptible to infection by B. bassiana in comparsion to the other fungal treatments, resulting in a high percentage of adult emerging. Similarly, Andreadis et al. (2016) reported that eggs and larvae of fungus gnats $L$. ingenua were ineffective to infection by $B$. bassiana when applied as pure fungal culture or into the growing medium. Stanghellini \& El-Hamalawi (2005) also demonstarted the larvae of shore flies S. stagnalis known as a vector of fungal pathogens in greenhouses were not highly sensitive to infection by $B$. bassiana as compared to adults.

Furthermore, $A$. strictum when applied as a pure fungal diet resulted in a high percentage of survival larvae compared to the other fungal strains. Survival rates of fungus gnat larvae were only affected by $A$. strictum, when the spore suspensions were mixed within the substarte, resulting in a reduced number of emerging adults. To the best of our knowledge, however, no studies have examined the direct effect of $A$. strictum on fungus gnat population. Further studies are warranted to determine the efficacy of this fungus on fungus gnat larvae in greenhouses.

In case of Bradysia spp., where larvae need to move through the substrate to find a food source, they might come into contact with spores applied via the suspension into the substrate, resulting in an increased probability of larvae to be infected with the fungal strain. EPF kill insects not only by penetrating through the cuticle, but also by producing toxic substances known as secondary metabolites which could infect insects (Vey et al., 2001). Samuels et al. (1998) reported 
that the EPF M. anisopliae might cause muscle paralysis, resulting in muscles weakness in infected insects and death. Furthermore, transferring larvae from stock-rearing medium, lack of suitable microbial food source and some intrinsic unidentified biological factors might reduce the emergence of adults (Olson et al., 2002, Main et al., 2014). The study of Wilkinson \& Daugherty (1970a) also indiacted that the weakened larvae and pupae might be cannibalized by fourth instars of larval fungus gnats in special conditions, which results in a reduction of adult emergence.

Although fungus gnat larvae feed on fungi as a food source (Kennedy, 1974, Anas \& Reeleder, 1988), some fungi are not attractive for fungus gnat larvae as a viable food source. For instance, fungus gnat larvae B. coprophila failed to feed on the fungus Trichoderma viride as a food source. The efficacy of fungal strains to the insect pests is variable, even among species of the same genus. For example in some cases, one fungus gnat species is highly attracted to a fungal strain (e.g. T. harzianum), while another species is not attracted (Frouz \& Naváková 2001).

In addition, it should be noted that the growing medium type and its components have an impact on the development and reproduction of fungus gnats (Jagdale et al., 2004, Cloyd \& Dickinson, 2008). This finding supports our study: the two substrates with different constituents used affected the development of larvae; however, the addition of fertilizer in these substrates did not provide a significant effect on the development of larvae as compared to the substrate without fertilizer. The substrate Fruhstorfer Erde Typ P used as the basic soil was more suitable for fungus gnat larvae as compared to the standard substrate (substrate 1, Klasmann-Deilmann), resulting a higher adult emergence. It might be due to the factors such as contents and activity of microbial colonies present in this substrate.

\section{Conclusion}

This study revealed that fungus gnat larvae are able to survive even on pure fungal diets or spore suspensions when are mixed with the substrate. Larval stage of fungus gnat was more susceptible to Metarhizium brunneum as compared to other fungal strains, resulting in a reduction of survival larvae and emergence rate of adults. Hence, M. brunneum was selected as an appropriate EPF for controlling fungus gnat larvae. This study could act as a framework for further studies on the application of M. brunneum to deal with fungus gnats. 


\section{Acknowledgements}

We would like to thank statistical center of Göttingen University and Khaldon Askoul for consulting the data analysis. We also would like to thank Bianca Tappe for their technical assistance. Finally, we thank Hadis Jayanti for reviewing this manuscript.

\section{References}

Acharya, R., Hwang, H. S., Shim, J. K., Yu, Y. S. \& Lee, K. Y. (2019) Control efficacy of fungus gnat, Bradysia impatiens, enhanced by a combination of entomopathogenic nematodes and predatory mites. Biological Control, 138.

Andreadis, S. S., Cloonan, K. R., Bellicanta, G. S., Paley, K., Pecchia, J. \& Jenkins, N. E. (2016) Efficacy of Beauveria bassiana formulations against the fungus gnat Lycoriella ingenua. Biological Control, 103, 165-171.

Anas, O. \& Reeleder, R. D. (1988) Feeding habits of Bradysia coprophila on fungi and plant tissue. Phytoprotection, 69, 73-78.

Bartlett, G. R. \& Keil, C. B. (1997) Identification and characterization of a permethrin resistance mechanism in populations of the fungus gnat Lycoriella mali (Fitch) (Diptera: Sciaridae). Pesticide Biochemistry and Physiology, 58, 173-181.

Cabrera, A. R., Cloyd, R. A. \& Zaborski, E. R. (2003) Effect of monitoring technique in determining the presence of fungus gnat, Bradysia spp. (Diptera: Sciaridae), larvae in growing medium. Journal of Agricultural and Urban Entomology, 20, 41-47.

Cloyd, R. A. (2000) Fungus gnat and shore fly management strategies: panel discussion, pp. 57-59. In: King, A. I. \& Greene, I. D. (eds.) Proceedings, the 16th Conference on Insect and Disease Management on Ornamentals. Society of American Florists, Alexandria, VA.

Cloyd, R. A. \& Zaborski, E. R. (2004) Fungus gnats, Bradysia spp. (Diptera: Sciaridae), and other arthropods in commercial bagged soilless growing media and rooted plant plugs. Journal of Economic Entomology, 97, 503-10.

Cloyd, R. A. \& Dickinson, A., (2006) Effect of Bacillus thuringiensis subsp. israelensis and neonicotinoid insecticides on the fungus gnat Bradysia sp nr. coprophila (Lintner) (Diptera: Sciaridae). Pest Management Science, 62, 171-177.

Cloyd, R. A. (2008) Management of fungus gnats (Bradysia spp.) in greenhouse and nurseries. Floriculture and Ornamental Biotechnology, 2, 84-89. 
Cloyd, R. A. \& Dickinson, A. (2008) Procedure to enhance the recovery rate of fungus gnat, Bradysia sp. nr. coprophila (Diptera: Sciaridae) adults from growing medium. HortScience, 43, 1528-1530.

Cloyd R. A. (2015) Ecology of fungus gnats (Bradysia spp.) in greenhouse production systems associated with disease-interactions and alternative management strategies. Insects, 6, 325332.

Dobrindt, L. (2013) INBIOSOIL Progress Report: Use of semiochemicals and entomopathogenic fungi for the biological control of fungus gnats.

Dreistadt, S. H. (2001) Fungus gnats, shore flies, moth flies, and march flies. In: Pest Notes. Publication 7448. University of California, Davis, CA.

El-Hamalawi, Z. A. (2008) Acquisition, retention and dispersal of soilborne plant pathogenic fungi by fungus gnats and moth flies. Annals of Applied Biology, 153, 195-203.

Filotas, M., Castrillo, L., Vandenberg, J., Sanderson, J. \& Wraight, S. (2005) Novel isolates of the entomopathogenic fungus Beauveria bassiana for biolpogical control of the shore fly, Scatella tenicosta. IOBC/WPRS bulletin, 28, 95-98.

Frouz, J. \& Naváková, A. (2001) A new method for rearing the sciarid fly, Lycoriella ingenua (Diptera: Sciaridae), in the laboratory: possible implications for the study of fly-fungal interactions. Pedobiologia, 45, 329-340.

Gardiner, R. B., Jarvis, W. R. \& Shipp, J. L. (1990) Ingestion of Pythium spp. by larvae of the fungus gnat Bradysia impatiens (Diptera:Sciaridae). Annals of Applied Biology, 116, 205-212.

Gillespie, D. R. \& Quiring, D. M. J. (1990) Biological control of fungus gnats, Bradysia spp. (Diptera: Sciaridae), and western flower thrips, Frankliniella occidentalis (Pergande) (Thysanoptera: Thripidae), in greenhouses using a soil-dwelling predatory mite, Geolaelaps sp. nr. aculeifer (Canestrini) (Acari: Laelapidae), Canadian Entomologist, 122, 975-983.

Gillespie, D. R. \& Menzies, J. G. (1993) Fungus gnats vector Fusarium oxysporum f.sp. radicislycopersici. Annals of Applied Biology, 123, 539-544.

Graham, C. L. \& McNeill, M. J. (1972) Soybean crown and root damage by Bradysia coprophila. Journal of Economic Entomology, 65, 597-599.

Hamlen, R. A. \& Mead, F. W. (1979) Fungus gnat larval control in greenhouse plant production. Journal of Economic Entomology, 72, 269-271.

Hungerford, H. B. (1916) Sciara maggots injurious to potted plants. Journal of Economic Entomology, 9, 538-549.

Jaber, L. R. \& Vidal, S. (2010) Fungal endophyte negative effects on herbivory are enhanced on 
intact plants and maintained in a subsequent generation. Ecological Entomology, 35, 25-36.

Jagdale, G. B., Casey, M. L., Grewal, P. S. \& Lindquist, R. K. (2004) Application rate and timing, potting medium, and host plant effects on the efficacy of Steinernema feltiae against the fungus gnat, Bradysia coprophila, in floriculture. Biological Control, 29, 296-305.

James, R. L., Dumroese, R. K. \& Wenny, D. L. (1995) Botrytis cinerea carried by adult fungus gnats (Diptera: Sciaridae) in container nurseries. Tree Planters' Notes, 46, 48-53.

Jallow, M. F. A., Dugassa-Gobena, D. \& Vidal, S. (2004) Indirect interaction between an unspecialized endophytic fungus and a polyphagous moth. Basic and Applied Ecology, 5, 183191.

Kennedy, M. K. (1974) Survival and development of Bradysia impatiens (Diptera: Sciaridae) on fungal and non-fungal food sources. Annals of the Entomological Society of America, 67, 745749.

Kennedy, M. K. (1976) The Interaction of Bradysia impatiens (Joh.) (Diptera: Sciaridae), a fungal host, and the root systems of vascular plants. PhD Dissertation, Cornell University, Ithaca, NY, USA.

Leath, K. T. \& Newton, R. C. (1969) Interaction of a fungus gnat, Bradysia sp. (Sciaridae) with Fusarium spp. on alfalfa and red clover. Phytopathology, 59, 257-258.

Lindquist, R. K., Faber, W. R. \& Casey, M. L. (1985) Effect of various soilless root media and insecticides on fungus gnats. HortScience, 20, 358-360.

Mercier, L. (1911) Sur le role des insectes comme agents de propagation de l'ergot des gramine'es. Comptes Rendus Socie'te' de Biologie (Paris), 70, 300-302.

Main, M., McCaffrey, J. P. \& Morra, M. J. (2014) Insecticidal activity of Brassica Juncea seed meal to the fungus gnat Bradysia impatiens Johannsen (Diptera:Sciaridae). Journal of Applied Entomology, 138, 701-7.

Osborne, L. S., Boucias, D. G. \& Lindquist, R. K. (1985) Activity of Bacillus thuringiensis var. israelensis on Bradysia coprophila (Diptera: Sciaridae). Journal of Economic Entomology, 78, 922-925.

Olson, D. L., Oetting, R. D. \& van lersel, M. W. (2002) Effect of soilless potting media and water management on development of fungus gnats (Diptera: Sciaridae) and plant growth. HortScience, 37, 919-923.

Samuels, R. I., Reynolds, S. E. \& Charnley, A. K. (1998) Calcium channel activation of insect muscle by destruxins, insecticidal compounds produced by entomopathogenic fungus Metarhizium 
anisopliae. Comparative Biochemistry and Physiology - Part C: Toxicology and Pharmacology, $90,403-412$.

Stanghellini, M. E. \& El-Hamalawi, Z. A. (2005) Efficacy of Beauveria bassiana on colonized millet seed as a biopesticide for the control of shore flies. HortScience, 40, 1384-1388.

Steffan, W. A. (1966) A generic revision of the family Sciaridae (Diptera) of America north of Mexico. University of California Publications in Entomology, 44, 1-77.

Strasser, H., Forer, A. \& Schinner, F. (1996) Development of media for the selective isolation and maintenance of virulence of Beauveria brongniartii. In: Jack-son, T. \& Glare, T., (eds.) Microbial Control of Soil Dwelling Pests. AgResearch, Lincoln, New Zealand, 125-130.

Tavoosi Ajvad, F., Madadi, H., Michaud, J. P., Zafari, D. \& Khanjani, M. (2019) Combined applications of an entomopathogenic fungus and a predatory Mite to control fungus gnats (Diptera: Sciaridae) in mushroom production. Biological Control, 141.

Vey, A., Hoagland, R. \& Butt, T. M. (2001) Toxic metabolites of fungal biocontrol agents. In: Butt, T. M., Jackson, C. W. \& Magan, N. (eds.) Fungi as biocontrol agents progress, problems and potential. CABI Publishing, Wallingford, UK, 311-346.

Watson, D. W., Geden, C. J., Long, S. J. \& Rutz, D. A. (1995) Efficacy of Beauveria bassiona for controlling the house fly and stable fly (Diptera: Muscidae). Biological Control, 5, 405-411.

Wilkinson, J. D. \& Daugherty, D. M. (1970a) The biology and immature stages of Bradysia impatiens (Diptera: Sciaridae). Annals of the Entomological Society of America, 63, 656-660. 
Chapter 2

Combining a biopesticide and a botanical compound against fungus gnat larvae Bradysia impatiens (Diptera: Sciaridae)

Mahsa Dehghani ${ }^{*}$, Jörg Rademacher and Stefan Vidal

* Corresponding author 


\title{
Combining a biopesticide and a botanical compound against fungus gnat larvae Bradysia impatiens (Diptera: Sciaridae)
}

M. Dehghani ${ }^{1 *}$, J. Rademacher ${ }^{2}$ and S. Vidal ${ }^{1}$

${ }^{1}$ Georg-August-Universität Göttingen, Department of Crop Sciences, Agricultural Entomology, Grisebachstrasse 6, 37077 Göttingen, Germany

${ }^{2}$ Katz Biotech AG, Baruth, Germany

*Corresponding author: E-mail address: mdehgha@gwdg.de

\begin{abstract}
Fungus gnats are regarded as pests, specifically in greenhouses and nurseries, due to the feeding of larvae on plant roots. Although various approaches for fungus gnats control are available, safer and more effective methods are desirable. The aim of this study was to determine the efficacy of biopesticides (Bacillus thuringiensis $\mathrm{Bt}$ and Metarhizium brunneum $\mathrm{Cb}$ 15) and botanical compounds for the control of fungus gnat larvae. In a first series of bioassays, Bt, at the recommended application rate, was mixed into the growing media supplemented with a botanical compound. $24 \mathrm{~h}$ post treatment (HPT), fungus gnat larvae were released into each experimental cup and adult emergence was evaluated two weeks later. In this experiment, Bt in combination with two formulations of the botanical compound (Minx and Mentha) resulted in an additive effect as shown by the reduced number of adults emerging. A higher percentage of fungus gnat adults emerged from larvae exposed to the botanical microcapsule Minx (63\%) than the control treatment, pointing to an attractant effect for the larvae. Subsequent bioassays were set-up to determine the efficacy of a combination of $M$. brunneum and the formulations of botanical compound for the control of fungus gnat larvae. Data collected from this trial demonstrated that significantly fewer adult numbers emerged from $M$. brunneum in combination with the botanical compound Minx (30\%) compared to the control (60\%) or the fungal treatment alone (33\%), pointing again to an additive effect of combining the two agents. These results pave the way for an "attract and kill" strategy, which could help to efficiently reduce fungus gnat populations.
\end{abstract}

Keywords: fungus gnats, Metarhizium brunneum, Bacillus thuringiensis, formulation, adult emergence 


\section{Introduction}

Fungus gnats of the genus Bradysia spp. (Diptera: Sciaridae) are ubiquitous pests in greenhouse crop-production systems. Fungus gnat larvae are mainly causing direct plant damage on the roots and stems (Wilkinson \& Daugherty, 1970a), disrupting water and nutrient uptake (Jarvis et al., 1993). Moreover, fungus gnats are able to transmit soil-borne plant pathogenic fungi such as Pythium spp., Fusarium spp., and Verticillium spp., from infected to healthy plants (Gillespie \& Menzie, 1993, Cloyd, 2015). In addition, adults are capable to vector the aerial conidia of plant pathogenic fungi, including Botrytis cinerea (James et al., 1995), Fusarium avenaceum (ElHamalawi \& Stangellini, 2005), Thielaviopsis basicola (Harris, 1995), and Verticillium albo-atrum (Kalb \& Millar, 1986) from infected to non-infected plants.

Insecticides are widely used as a drench application to the growing medium of the plants for the control of larval fungus gnats (Hamlen \& Mead, 1979, Cloyd \& Dickinson, 2006). However, the continuous use of synthetic insecticides has resulted in the development of resistance and in harmful effects on non-target organisms, human and environment (Smith \& Stratton, 1986, Cloyd, 2008). Several biological control agents have been reported to be effective for managing fungus gnats such as the predatory mite Hypoaspis miles, a rove beetle (Dalotia coriaria), and entomopathogenic nematodes (Steinernema feltiae) (Chambers et al., 1993, Harris et al., 1995, Cloyd \& Dickinson, 2006). Furthermore, biologically based insecticides have been used to keep fungus gnat population below the economic threshold level. A common property of these biological insecticides, mainly derived from fungi or bacteria, is their potential to produce toxins against insect pests (Quesada-Moraga et al., 2006).

Among biorational insecticides, Bacillus thuringiensis Berliner subsp. israelensis (Bt) was found to suppress fungus gnat populations in greenhouse production systems (Osborne et al., 1985). Bt is a gram-positive soil bacterium and able to produce crystalline inclusions, which release insecticidal crystal proteins when ingested by insects. Most of these crystal proteins are transformed into the smaller toxic polypepetids in the midgut and interact with the midgut epithelium cells of insects (Höfte \& Whiteley, 1989). Various formulations of Bt have been used commercially as biopesticide to control agricultural pests. One of these commercial Bt products is BioMükk ${ }^{\circledR}$ WDG, which is used in this study. Due to the short residual activity of $\mathrm{Bt}$, it needs to be applied repeatedly to provide a long-term control (Osborne et al., 1985). The application of the microbial insecticide Bt for the control of fungus gnats was first evaluated by Osborne et al. (1985), who observed a high effectiveness of Bt against $1^{\text {st }}$ and $2^{\text {nd }}$ instars of fungus gnat larvae. 
Moreover, the high efficacy of Bt against fungus gnats was reported by Harris et al. (1995), while some studies demonstrated that Bt was ineffective against larval fungus gnats (Cloyd \& Dickinson, 2006, Shamshad et al., 2008, Koller, 2011).

The present study builds upon the results of a previous study, which reports a high efficacy of the entomopathogenic fungus Metarhizium brunneum as a microbial control agent against larval fungus gnats, either in pure diet or applied into the growing medium. M. anisopliae (Metschnikoff) Sorokin from the order Hypocreales (Ascomycota) is one of the most commercially entomopathogenic fungi (EPF) that has been reported against wide range of insects including Diptera, e.g. mosquitos (Culicidae) (Paula et al., 2018) and Sciaridae (Tavoosi Ajvad et al., 2019). Baker et al. (2018) demonstrated that EPFs (M. anisopliae) are slow-killing agents, causing a reduction in the reproductive potential of insects (e.g. house fly, Musca domestica). Metarhizium spp. produces small molecules including destruxins (DTXs), which cause negative effects on insects including paralysis (Samuels et al., 1988), inhibition DNA and RNA synthesis (Quiot et al., 1985), suppression of defense response of insects (Golo et al., 2014). The use of EPFs (entomopathogenic fungi) for pest control has received commercial attention, due to the technical aspects such as mass production of propagules and sufficient efficacy, the reasonable costs, long-term stability and consistent efficacy to infect insects (Vega et al., 2008). Tavoosi Ajvad et al. (2019) reported a high susceptibility of larval fungus gnats Lycoriella auripilla Winnertz to infection by M. anisopliae.

A combined application of biological agents has been recently developed and evaluated for the control of insect pests, which may lead to enhanced control efficiency, simultaneously reducing the amount of active ingredients and thus decreasing the risk of environmental pollution and pest resistance (Quintela \& McCoy, 1998). For instance, the entomopathogenic fungus $M$. anisopliae in combination with a predatory mite (Gaeolaelaps aculeifer) increased fungus gnat ( $L$. auripilla) mortality in mushroom production (Tavoosi Ajvad et al., 2019). An additive effect has been also observed by Acharya et al. (2019) for the combined application of an entomopathogenic nematode EPN (Heterorhabditis indica) and the predatory mites (Stratiolaelaps scimitus) against fungus gnat larvae (B. impatiens).

We hypothesized that increased control efficiency could be realized by a combination of an attractant with an entomopathogenic fungus in an "attract and kill" strategy. El-Sayed et al. (2009) demonstrated that using this strategy an insect pest is lured towards an attractive agent, and is thus more exposed to a killing agent. In our study, a combination of an EPF (e.g. M. brunneum) with a biological product (e.g. botanical compound) was evaluated for this new strategy to control 
fungus gnats. Hence, the objectives of this research were: (1) to evaluate the effect of two different formulations of botanical compound for fungus gnat larvae and (2) to determine the efficacy of microbial insecticides (Metarhizium brunneum strain $\mathrm{Cb} 15$ and Bacillus thuringiensis, Bt) individually or in combination with the botanical compounds against fungus gnat larvae and whether these combined formulations are able to enhance control efficiency of fungus gnats.

\section{Materials and methods}

\section{Fungus gnat larvae}

The fungus gnat species $B$. impatiens used in this study was obtained from a colony maintained in Agricultural Entomology Section, Crop Science Department, Georg-August Universität Göttingen, Germany. To initiate egg laying, a plastic tray was filled with a mixture of substrate (350 g) (Klasmann-Deilmann GmbH; Geeste, Germany), oat meal (3 gr) and shredded potato. These plastic containers were placed into the permanent colonies and left there for 2 days to allow the females to oviposit. After 2 days, the plastic trays were removed from the permanent colonies and placed into a room at $23 \pm 2^{\circ} \mathrm{C}$ with a 16-h photoperiod until third instar larvae emerged. The newly hatched third instar larvae were collected with a cooked potato disk placed on the surface of the substrate (Cabrera et al., 2003). Larvae were isolated via a floating technique. For all experiments, the newly emerged third instar larvae were used.

\section{Treatment preparation and application}

"Fungal preparation": The fungal strain used for this study, M. brunneum (Cb 15), is maintained at the Agricultural Entomology section, Georg-August Universität Göttingen, Germany. This fungal strain was cultured on potato extract glucose agar (PDA; Carl Roth ${ }^{\circledR}$, Germany) for ca. 3 weeks and incubated at $25 \pm 1^{\circ} \mathrm{C}$ in a climate chamber (Biologischer Klimaschrank WB 750, Mytron Bio- und Solartechnik $\mathrm{GmbH}$, Heilbad Heiligenstadt, Germany). The fungal suspension was prepared by scraping the surface of the Petri plate into a glass beaker including $50 \mathrm{ml}$ sterile demineralized water plus Tween 80 (0.1 \% v/v; Carl Roth ${ }^{\oplus}$, Germany). The conidial suspension was filtered through a fine mesh to remove mycelium. The spore suspension was verified with light microscopy (200X magnification, BH2-HLSH, Olympus Corporation, Tokyo, Japan). This pure fungal suspension used in the experiments was adjusted by diluting the conidia with demineralized water to a final concentration of $1 \times 10^{7}$ spores $/ \mathrm{ml}$. The number of spores was counted using a Neubauer 
Hemocytometer (0.1 mm depth) (Thoma CE, Paul Marienfeld GmbH \& Co.KG, Lauda-Königshofen, Germany).

"Botanical compounds": Two botanical products were obtained from Katz Biotech AG; Baruth, Germany and stored in a cooling chamber $\left(6^{\circ} \mathrm{C}\right)$ until use. Mint oil was embedded in capsules with two different formulations and components (Minx and Mentha). The composition of the botanical capsule (Minx) was made up of vegetable oil (approximately 50\% dry matter), (Na)-alginate solution, silica gel, mint oil and water ( $5 \%$ in dry matter). These components were suspended in a Na-alginate solution and this suspension was dripped with a syringe through droplet capillaries into $\mathrm{a} \mathrm{CaCl}_{2}$ solution, where the droplets were solidified by a gelation forming a hydrogel. The solid alginate capsule was dried in a fluid bed. A solid form of this botanical capsule was used in this study (J. Rademacher, personal communication, Katz Biotech AG; Baruth, Germany). The main advantage of the Minx Capsule is the simple preparation of this capsule; thus it could be easily manufactured at large scale (Fig.1).

No information is yet available about the preparation of the granule formulation of the botanical compound (Mentha). The preparation of this compound was reported costly as compared to microcapsule formulation.
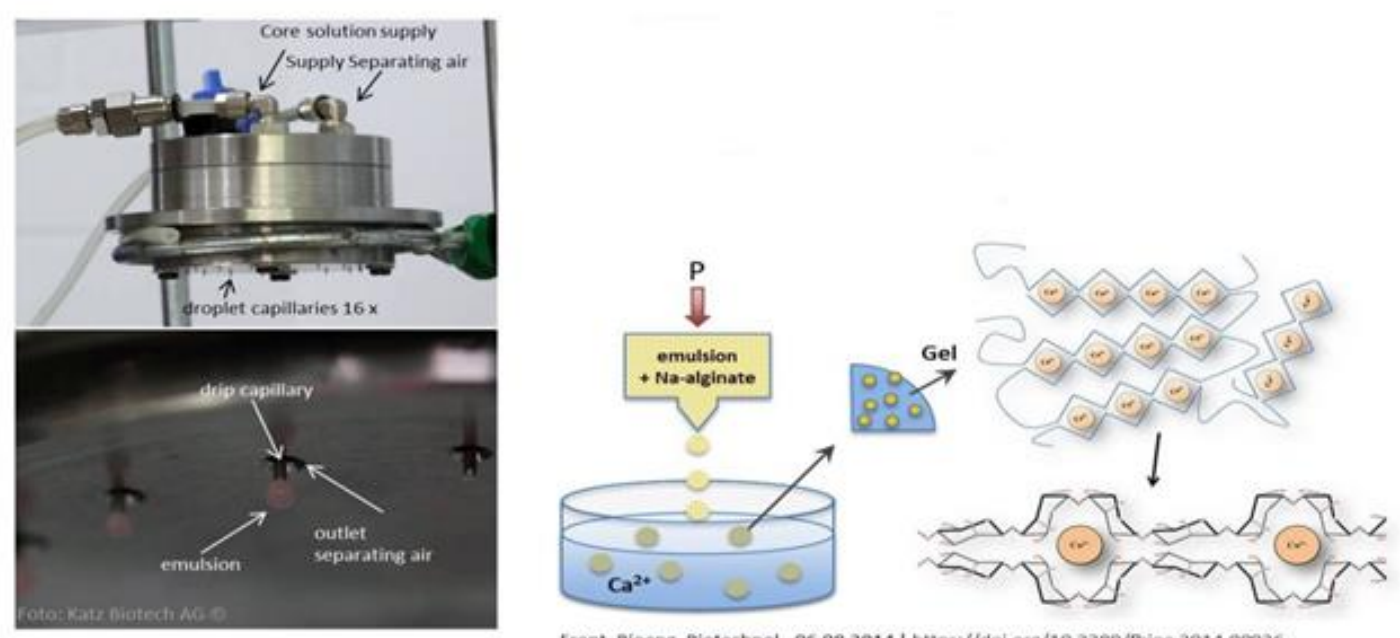

Front. Bioeng, Biotechnol., 06.08.2014 | bttos://doi.arg/10.3389/7bioe.2014.00026

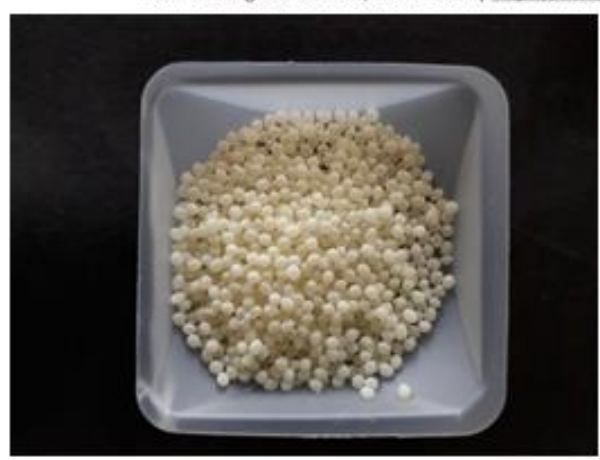

Fig. 1: Structure of the alginate matrix capsule (Minx) used in this study (Katz. Biotech $\mathrm{AG}^{\circledR}$ ). 


\section{Experimental design}

\section{Experiment 1}

The following experiment was designed to identify the efficacy of Minx capsules on the fungus gnat larvae. In this experiment, Minx microcapsules at different rates (5, 10, 15, 20, $50 \mathrm{mg})$ were placed individually on water agar (Agar-Agar, Kobe I, Carl Roth GmbH \& Co. KG, Karlsruhe, Germany) plates. One fungus gnat larva was transferred to the opposite site of the Minx capsules on the water agar using a fine brush and plates were incubated in a growth chamber at $25 \pm 1^{\circ} \mathrm{C}$ and $65 \pm 5 \%$ RH (Biologischer Klimaschrank WB 750, Mytron Bio- und Solartechnik GmbH, Heilbad Heiligenstadt, Germany). One hour after the release of a larva, the movement and activity of was monitored under a binocular microscope (ZEISS, Carl Zeiss Microscopy GmbH, Jena, Germany) to check whether Minx capsules are attractive for fungus gnat larvae. The activity of fungus gnat larvae was observed over a 24-h period. Each treatment was replicated three times.

\section{Experiment 2}

This study was conducted to assess the efficacy of the entomopathogenic fungi (EPF) and a botanical compound on fungus gnat adults using multiple-choice experiments. The multiple-choice (olfactometer system) involved the use of four-armed experimental arenas, which included a central compartment made from a clear, rectangle polypropylene container $(11 \times 8.5 \mathrm{~cm}$, Nette Papier $\mathrm{GmbH}$, Göttingen, Germany). In addition, the rectangle sample container $(10 \times 7.5 \mathrm{~cm})$ containing $100 \mathrm{ml}$ substrate (Fruhstorfer Erde Typ P 25) was positioned directly across from each other and the arms associated with the retaining four experimental arenas were made from the clear polypropylene tube $(4.0 \mathrm{~cm}$ length). The plastic containers used in this study did not emit volatiles (as described by Cloyd et al., 2007a). A four-armed experimental arena was treated with the four treatments: Bt at the recommended application rate as mentioned in experiment $1(0.03$ g BioMükk ${ }^{\circledR}$ WDG in $7.5 \mathrm{ml}$ water per sample container), a botanical compound (Minx) individually ( $2 \mathrm{mg}$ ) or in combination with $7.5 \mathrm{ml}$ fungal strain of $M$. brunneum $\mathrm{Cb} 15$ at a rate of $1 \times 10^{7}$ spore/ml and a control. Control substrates were treated with sterilized water with the same volume as the biopesticide treatment. Each compartment was covered with a lid containing a yellow sticky card to capture adults. Forty fungus gnat adults (mixture of females and males; sex ratio about $50 \%$ ) were released in the central compartment of each experimental arena. $48 \mathrm{~h}$ after 
the release of fungus gnat adults, the number of captured adults per yellow sticky card fixed at the top per treatment was recorded replicated six times.

\section{Experiment 3}

The efficacy of Bt alone and in combination with two different formulations of the botanical compounds for the control of fungus gnat larvae was evaluated. A clear plastic cup ( $300 \mathrm{ml}$, Nette Papier GmbH, Göttingen, Germany) was filled with $250 \mathrm{ml}$ substrate (Fruhstorfer Erde Typ P 25; Hawita Gruppe $\mathrm{GmbH}$, Vechta, Germany). In each plastic cup with $10 \mathrm{~cm}$ diameter, the recommended rate of $\mathrm{Bt}$ BioMükk ${ }^{\circledR}$ WDG 300 - 400 g / 100 m2 in 1 L / 1 m2 water (Biofa; Münsingen, Germany) (0.032 g / $78.5 \mathrm{~cm} 2$ in $7.85 \mathrm{ml}$ water per cup) was applied directly to the substrate treated with the botanical microcapsule Minx (5 mg) or Mentha (1 mg). After 24h, 20 newly moulted third instar larvae were transferred into each experimental cup, which were stored in a climatic chamber (Biologischer Klimaschrank WB 750, Mytron Bio- und Solartechnik GmbH, Heilbad Heiligenstadt, Germany) (photoperiod: $16: 8 \mathrm{~h}$; air temperature and humidity: $25 \pm 1^{\circ} \mathrm{C}, 65$ $\pm 5 \%)$ to complete their life cycle to adults. At the top of each cup, a yellow sticky card was adhered to monitor the number of fungus gnat adults over a period of two weeks. Each cup was closed with a lid, which was punctured with some holes to allow air circulation. Each treatment was replicated 10 times (experimental cups), totaling 80 cups. The control cups consisted of substrate treated with water.

\section{Experiment 4}

In this bioassay, we assessed the combination of $M$. brunneum ( $\mathrm{Cb}$ 15) and botanical compounds against fungus gnats. This trial was similar to experiment 3, except that $10 \mathrm{ml}$ of the $M$. brunneum ( $\mathrm{Cb}$ 15) spore suspensions were drench-applied at a rate of $1 \times 10^{7} \mathrm{spore} / \mathrm{ml}$ directly to the substrate (Fruhstorfer Erde Typ P 25; Hawita Gruppe GmbH, Vechta, Germany) treated with the botanical microcapsules Minx (5 mg) or Mentha (1 mg) individually. In addition, M. brunneum suspensions were applied to the substrate treated with $\mathrm{Bt}$ at the same rate mentioned as in experiment 3 . These treatments were mixed within substrate. $24 \mathrm{~h}$ after treatment, 20 third instar fungus gnat larvae were transferred into each cup and maintained in a climatic chamber as described in trial 3 . Control cups received $10 \mathrm{ml}$ sterilized water. Fungus gnat adult emergence was assessed over a period of two week using a yellow sticky $(2.5 \times 2.5 \mathrm{~cm})$ attached at the top of each cup to trap the emerging adults. A total of 10 replications for each treatment were set up. 


\section{Experiment 5}

The effectiveness of combining an EPF (M. brunneum) and the botanical compounds (Minx and Mentha) was tested in four growing media with different constituents against fungus gnat larvae. Each plastic cup (300 ml, Nette Papier GmbH, Göttingen, Germany) was filled with $250 \mathrm{ml}$ different substrates including Fruhstorfer Erde Typ P, T and Null (Hawita Gruppe GmbH, Vechta, Germany), and the standard substrate (Substrate 1: Klasmann-Deilmann GmbH Geeste, Germany). $10 \mathrm{ml}$ of the fungal suspension of $M$. brunneum was added at a rate of $1 \times 10^{7} \mathrm{spore} / \mathrm{ml}$ directly to the substrate treated with the botanical compound Minx $(5 \mathrm{mg})$ or Mentha $(1 \mathrm{mg})$. Control cups were treated with $10 \mathrm{ml}$ sterilized water. 24h after treatment (HPT), twenty newly hatched third instar larvae were released into each experimental cup and incubated in a climatic chamber at $25 \pm 1^{\circ} \mathrm{C}$ and $65 \pm 5 \% \mathrm{RH}$ with a photoperiod 16:8 h (Biologischer Klimaschrank WB 750, Mytron Bio- und Solartechnik $\mathrm{GmbH}$, Heilbad Heiligenstadt, Germany). Each cup was closed with a lid and covered using a yellow sticky card fixed at the top to trap the emerging adults. After two weeks, the total numbers of emerging adults in each cup was counted. This experiment was arranged with ten replications per treatment.

\section{Data analysis}

All statistical analysis was performed with $\mathrm{R}$ version 3.5.3. Graphs were created with $\mathrm{R}$ (experiment 2, 3, 4) and SigmaPlot, version 11.0 (experiment 5) (Analytical Software, Tallahasee, FI, USA). Statistical analysis was performed using GLM with quasipoisson distribution to determine the efficacy of two biopesticides (Bt and M. brunneum) when applied alone or in combination with the botanical compounds against fungus gnat larvae (experiment 3 and 4). In experiment 5, GLM with quasipoisson distribution was used to analyze the efficacy of $M$. brunneum in combination with the botanical compounds for the control of fungus gnats in the four different substrates. In addition, the mean numbers of fungus gnat adults captured on the yellow sticky card as independent variable were calculated as percentage and were analyzed using one-way ANOVA (experiment 2). All data were first checked for normality and error variance of homoscedasticity. The significant differences between treatments were determined using the Tukey Honestly test at $\mathrm{P}<0.05$ 


\section{Results}

\section{Experiment 1}

In this trial, one hour after the release of fungus gnat larvae on water agar, they moved directly towards the Minx capsules, which were placed on the opposite site of the starting point, exhibiting an attractant effect for fungus gnat larvae (Fig. 2). According to the binocular observations, fungus gnat larvae are attracted to Minx capsules, but they do not feed on these capsules. In some plates, it was observed that after a certain time period, they move away and seemed to search for another food source.

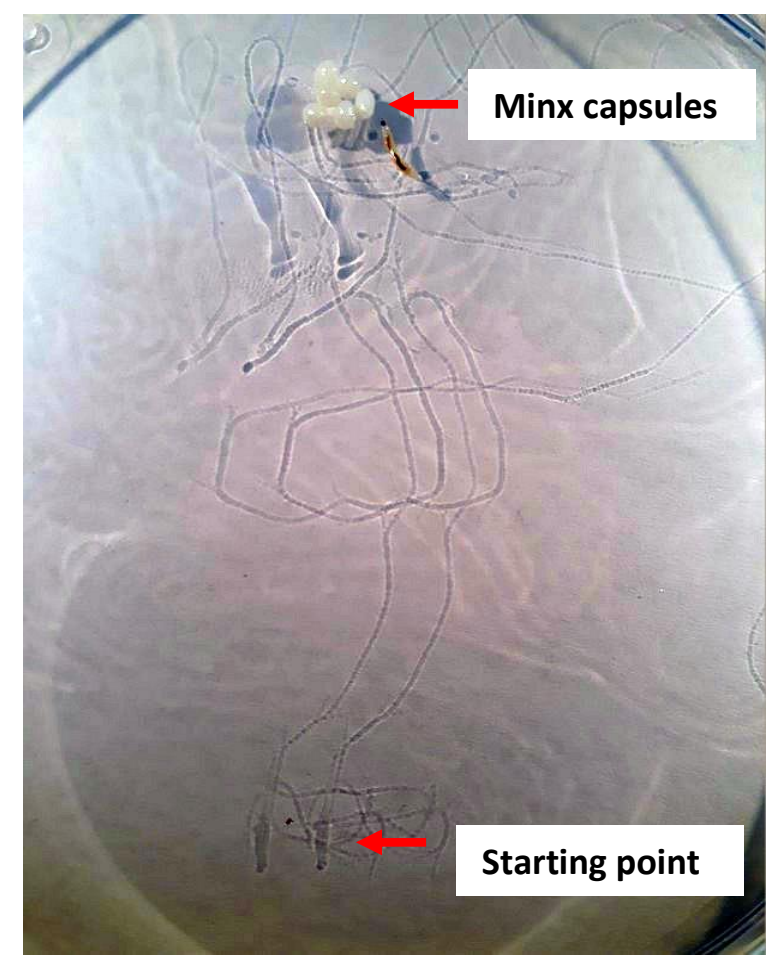

Fig. 2: straight movement of fungus gnat larvae towards the Minx capsules.

\section{Experiment 2}

There was a high variation in the number of captured fungus gnat adults among treatments tested in this study. The mean number of fungus gnat adults was significantly attracted to the sample compartment with the botanical compound Minx (36.6\%) in comparison to the other treatments $\left(F_{4,25}=9.83, p<0.001 ;\right.$ Fig. 3$)$. The mean numbers of captured fungus gnat adults in the sample compartments associated with M. brunneum in combination with Minx (21.25\%) and control treatment $(22.08 \%)$ were lower than $\operatorname{Minx}$ alone $\left(F_{2,15}=4.55, p<0.05\right)$. Moreover, sample compartments containing Bt (12.91\%) were less attractive to fungus gnat adults compared to the 
other treatments. The mean number of fungus gnat adults present in the central compartment was found $7.83 \%$.

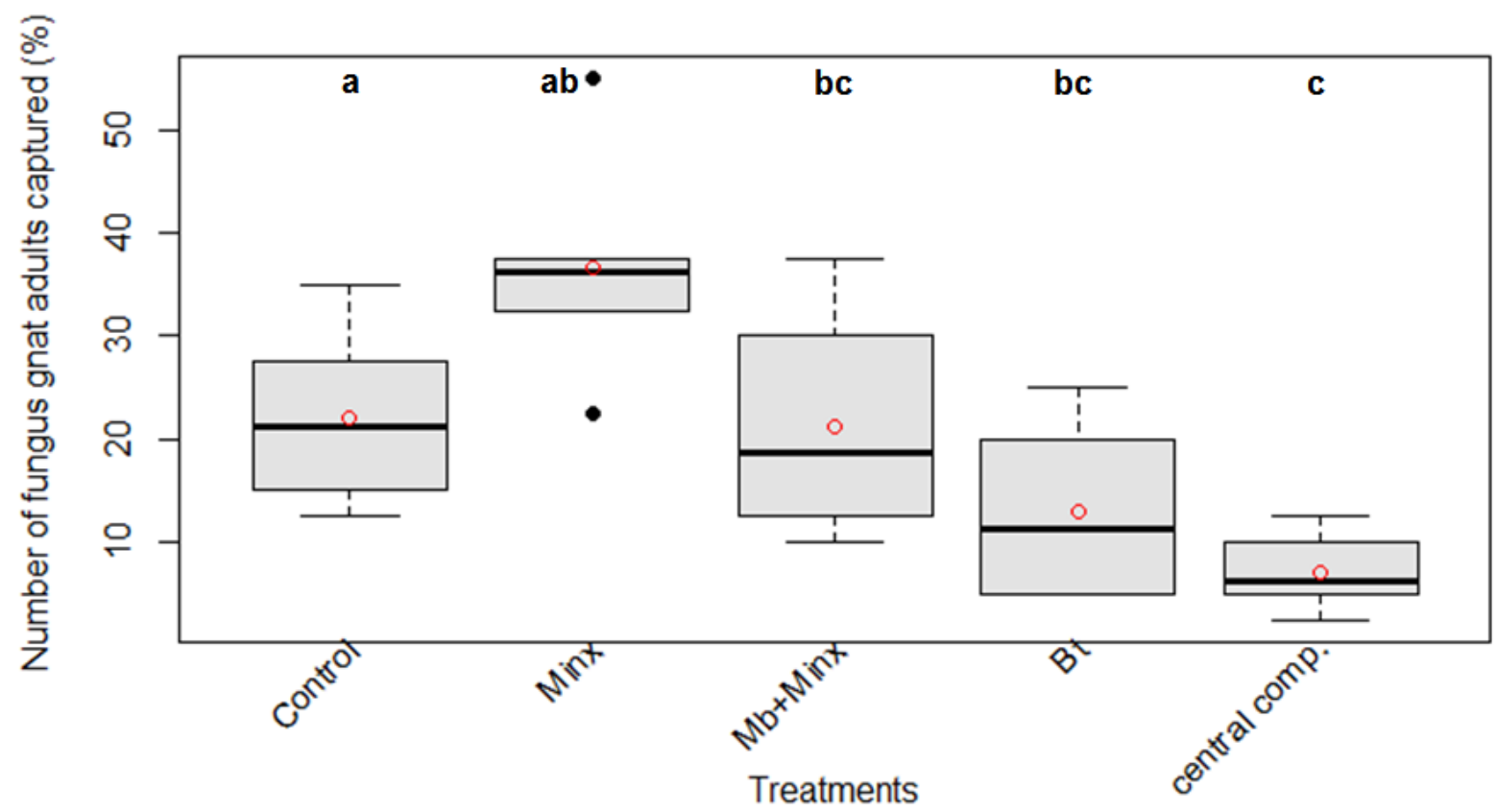

Fig. 3: Percentage of captured fungus gnat adults in the sample compartments associated with $M$. brunneum in combination with Minx (Mb + Minx) or B. thuringiensis (Bt) (BioMükk ${ }^{\circledR}$ WDG). The mean number of fungus gnat adults captured in the sample compartment was calculated as a percentage. The different letters indicate significant differences between treatments (Tukey HSD test) $\left(F_{4,25}=9.83, p<0.05\right) . *[$ Central comp. $=$ Central compartment $]$

\section{Experiment 3}

The application of Bt alone or in combination with the botanical compounds (Minx and Mentha) reduced the adult emergence in comparison to the control $\left(F_{6,63}=2.13, p=0.0615 ;\right.$ Fig. 4). The percentage of adult emergence from substrates treated with $\mathrm{Bt}$ in combination with botanical capsules, Minx $37 \%$ and Mentha $32.5 \%$, was lower than the emergence of adults from Bt alone $42 \%$. In contrast, the highest percentage of fungus gnat adults was observed in the substrate treated with Minx (63\%), followed by control and Mentha treatments (60\% and $49.5 \%$, respectively), although there were no significant differences between Minx and control treatments $\left(F_{1,18}=0.07, p=0.8\right)$. Therefore, Minx capsule exhibited an attractant effect for the larvae. The combination of three treatments $(B t+\operatorname{Minx}+$ Mentha $)$ resulted in high adult emergence $59.9 \%$, with no significant differences between this treatment and control $\left(\mathrm{F}_{1,18}=\right.$ $0.003, p=0.95)$. 


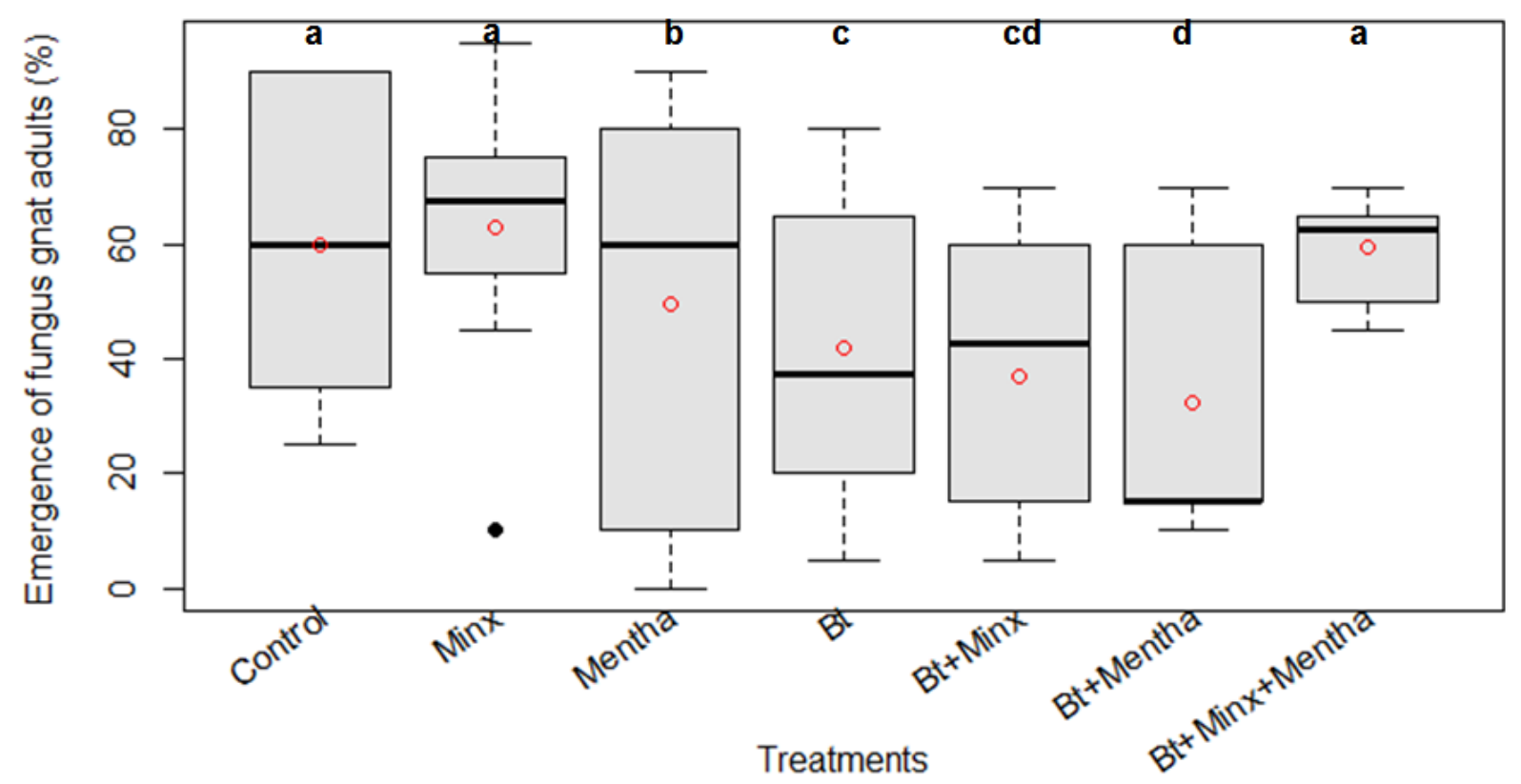

Fig. 4: Emergence rate of fungus gnat adults (\%) hatching from larvae exposed to Bacillus thuringiensis alone (Bt) or in combination with the botanical capsules Minx and Mentha. The different letters indicate significant differences between treatments (Tukey HSD test) $\left(F_{6,63}=2.13\right.$, $p=0.0615)$.

\section{Experiment 4}

The number of emerged adults differed significantly among treatments. The amount of fungus gnat adults hatching from $M$. brunneum $\mathrm{Cb} 15$ alone or in combination with botanical compounds was significantly lower than the control treatment $\left(F_{4,45}=12.24, p<0.001\right.$; Fig. 5). An efficient reduction of adult emergence was also found in the substrates treated with $M$. brunneum in combination with Minx (30\%) or Bt (22.5\%) as compared to $\mathrm{Mb}$ alone (33\%) or in combination with Mentha (37.5\%). The percentage of adult emergence from substrates treated with $M$. brunneum in combination with Mentha was significantly reduced in comparison to the control treatment, but this combination had no additive effect for reducing the adult emergence as compared to the combination of M. brunneum and Minx capsule. This effect was not significant $\left(F_{1,18}=6.63, p>0.01\right)$. 


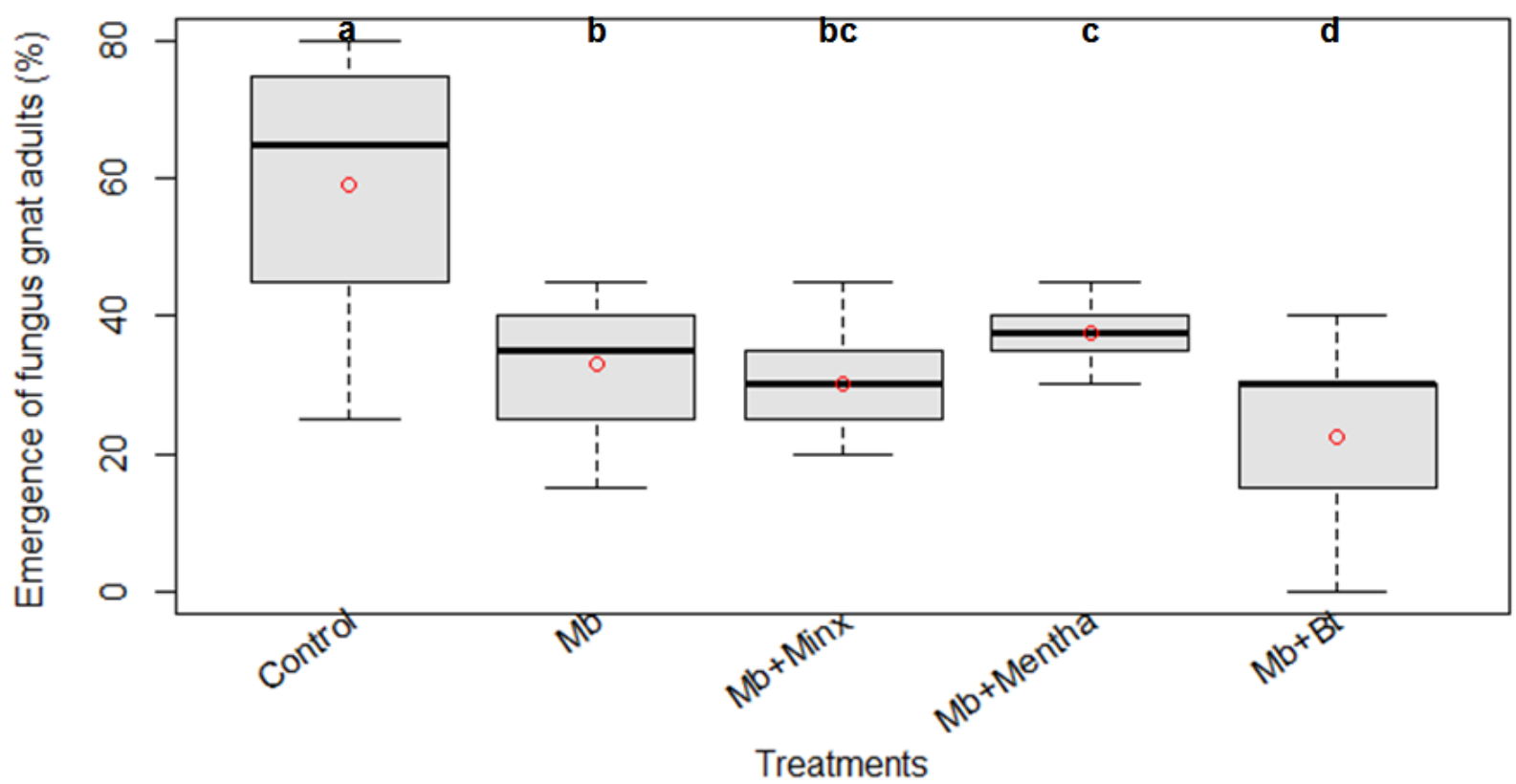

Fig. 5: Emergence of fungus gnat adults (\%) from larvae treated with $M$. brunneum (Mb) alone and in combination with two botanical compounds (Minx and Mentha) or B. thuringiensis (Bt) (BioMükk ${ }^{\circledR}$ WDG). The different letters indicate significant differences between treatments (Tukey HSD test) $\left(F_{4,45}=12.24 p<0.05\right)$.

\section{Experiment 5}

M. brunneum $(\mathrm{Mb})$ treatments individually or in combination with two botanical compounds significantly reduced the adults emergence in the four different substrates compared to the control $\left(F_{15,143}=9.56, p<0.001 ; F i g .6\right)$. The emergence of adults in the $M$. brunneum treatments, when applied alone or in combination with two botanical compounds, ranged from $18.3 \%$ (Mb + Minx in the substrate Typ $\mathrm{P})$ to $27.5 \%$ ( $\mathrm{Mb}+$ Mentha in the standard substrate), with no significant differences between treatments $\left(F_{11,107}=0.58, p=0.83\right)$. Among these treatments, $M$. brunneum in combination with Minx caused a high efficacy in the reduction of adults emergence in the substrates Fruhstorfer Erde Typ P (18.3 \%), Typ T (20.5 \%) and Typ Null (19.5 \%) as compared to the control substrates $\left(\mathrm{F}_{5,53}=17.97, \mathrm{p}<0.001\right)$. Indeed, in these three substrates, $\mathrm{Mb}$ in combination with Minx exhibited an additive effect as shown by the reduced number of adult emergence in comparison to the Mb alone (Substrates Fruhstorfer Erde Typ P: 23.5 \%; Type T: 22.5 \%; Typ Null: $22.5 \%$ ). This additive effect was not observed in the standard substrate containing $\mathrm{Mb}$ in combination with Minx (26.5\%), although this effect was not significantly, when compared to the $\mathrm{Mb}$ alone (25.5\%). Additionally, $\mathrm{Mb}$ in combination with Mentha reduced the number of 
fungus gnat adults compared to the control treatments in all substrates $\left(F_{7,72}=12.9, p<0.001\right)$, but this combination did not show an efficient reduction of fungus gnat adult emergence as compared to the combination of $\mathrm{Mb}$ with Minx capsules. However, this effect was not significant $\left(F_{7,71}=1.08\right.$, $p=0.38)$.

The data from this experiment also indicated that the different types of substrates did not significantly influence adult emergence. Among all substrates, the number of emerging adults from the substrate Fruhstorfer Erde Typ T (63\%) was higher, although not significantly, than the other substrates $\left(F_{3,36}=0.96, p=0.42\right)$.

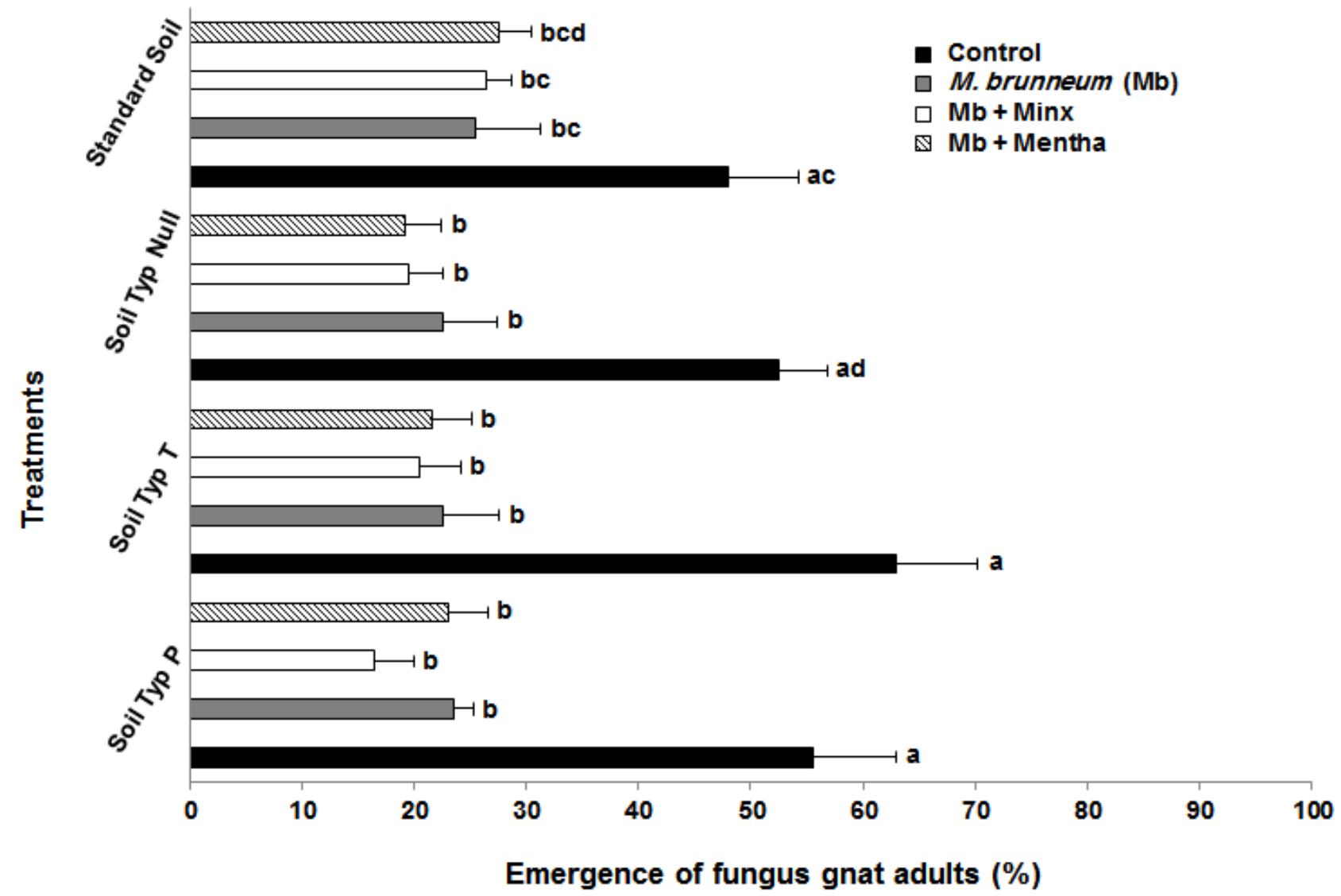

Fig. 6: Number of fungus gnat adults (\%) (mean $\pm \mathrm{SE}$ ) emerged from larvae exposed to the $M$. brunneum (Mb) individually and in combination with two botanical compounds (Minx and Mentha) in four different substrates. The different letters indicate significant differences between treatments (Tukey HSD test) $\left(F_{15,143}=9.56, p<0.05\right)$. 


\section{Discussion}

This study aimed to determine the efficacy of combining a biopesticide and a botanical compound in an "attract and kill" strategy targeting fungus gnat larvae. The combination of the biopesticides B. thuringiensis $\mathrm{Bt}$ or $\mathrm{M}$. brunneum $(\mathrm{Mb})$ with the botanical capsules Minx caused an additive effect, resulting in a reduction of adults emergence by $63 \%$ and $70 \%$, respectively. Specifically, the combination of $M$. brunneum and the botanical capsule Minx resulted in an additive impact as shown by the reduced number of emerging adults as compared to the single fungal treatment or other combinations. In addition, a higher percentage of adults emerged from larvae exposed the botanical capsule Minx alone, indicating a positive interaction of larvae with this capsule type. Adult fungus gnats also exhibited a strong attraction to the botanical capsules Minx. Therefore, Minx capsules were chosen for further trials due to its attractant properties and for the cheaper price to prepare it by a manufacturer. Additionally, the combined application of biopesticides and the botanical compound, formulated in a slow release capsule might offer a new strategy e.g. an "attract and kill" strategy for the control of fungus gnat larvae.

Our study showed a low susceptibility of third instar fungus gnat larvae to infection by $B$. thuringiensis Bt (BioMükk ${ }^{\circledR}$ WDG). This low efficacy of Bt was also shown by Molloy et al. (1981), who demonstrated that the earlier instar larvae $\left(1^{\text {st }}\right.$ and $\left.2^{\text {nd }}\right)$ are more susceptible to infection by Bt than later instars. This might be due to the fact that the later instars larvae need to consume more bacterium than the earlier instars of larvae to be negatively affected (Osborne et al., 1985). This reason was also suggested by Cloyd \& Dickinson (2006), which found that Bt was ineffective against second and third instar larvae of the fungus gnat B. coprophila. Margalit et al. (1985) also reported a short residual activity of $\mathrm{Bt}$ in the substrate due to its adsorption to the substrate particles, which might lead to an immediate reduction in toxicity of $\mathrm{Bt}$, although the bacterium remains viable for three weeks (Ohana et al., 1987). The study of Shamshad et al. (2008) indicated that $\mathrm{Bt}$ had no significant effect on the emergence of fungus gnat $L$. ingenua in mushroom production. Similarly, Jess \& Kilpatrick (2000) also found that Bt was ineffective in controlling the mushroom sciarid, L. ingenua.

Minx capsules have been found to be excellent attractants for fungus gnat larvae of $B$. impatiens. The laboratory experiments using water agar (experiment 1 ) showed the larval fungus gnat moves straightly towards the Minx capsules and remains a certain time around of this botanical capsule to find a food source, but larva moved away later from the Minx capsules (personal observation). We hypothesized that the simultaneous application of a biopestcide (e.g. 
Bt or M. brunneum) with an attractive agent (= Minx capsules) might increase fungus gnat control efficiacy. Moreover, volatiles generated from Minx capsules applied in the substrate have been shown as an attractive stimulant for fungus gnat adults, resulting in a higher number of individuals captured in the sample compartment containing Minx (experiment 2). In this regard, Meers \& Cloyd (2005) suggested that volatiles emitted from growing medium play a pivotal role in attraction and ovipositional preference of fungus gnat adults. For instance, the study of Guerenstein \& Hildebrand (2008) revealed that $\mathrm{CO}_{2}$ as a metabolic by-product could be an attractant for some Nematoceran Diptera e.g. mosquitoes. The attractiveness of $\mathrm{CO}_{2}$ derived from litter of mushroom cultures to fungus gnats $L$. ingenua was also demonstrated by Frouz et al. (2002).

The combined application of Bt and botanical capsules exhibited a slightly higher reduction of adult emergence, although not significantly, as compared to $\mathrm{Bt}$ alone. This reduction might be due to the emigration of fungus gnat larvae towards the botanical capsules (Minx) as an attractive agent in the growing medium, thus enhancing the probability of the fungus gnat larvae to be negatively affected by Bt. Therefore; this combined formulation might cause a better efficacy to control fungus gnats. However, Osborne et al. (1985) indicated that the second and third instars of larvae (later instars) need to consume more bacterium to be negatively influenced by $\mathrm{Bt}$ as compared to the earlier instars. Hanitzsch (2015) demonstrated a higher efficacy of co-formulated Bt than unformulated Bt against fungus gnat larvae. Given these results, we extended the use of the combined formulations for the control of fungus gnats Bradysia spp. using EPF (e.g. M. brunneum).

M. brunneum has been shown to be effective (67\%) in reducing adult emergence of fungus gnats. Tavoosi Ajvad et al. (2019) obtained > 50 \% larval mortality of L. auripilla using EPF M. anisopliae. Andreadis et al. (2016) achieved L. ingenua pupae (41\% mortality) and adults (100\% mortality) were susceptible to infection by another species of EPF e.g. Beauveria bassiana. We found an additive effect of two biological agents ( $M$. brunneum and botanical capsules) for the control of fungus gnats Bradysia spp., resulting in an enhanced control efficacy of fungus gnats as shown by the reduced number of adults emerging ( $70 \%)$, although not significantly, compared to the individual fungal treatment (67\%). We suppose that the botanical capsule (Minx) increases the movement and activity of the fungus gnat larvae, resulting in an increase in the possibility of larvae to come in contact with the spores mixed in the substrate. Therefore, the combined 
application might lead to a better efficacy to control fungus gnat larvae, although this idea needs further testing.

This is the first study that examined the effects of combined application of M. brunneum and a botanical compound against fungus gnat larvae. There have been some previous reports on the combined application of two biological agents against fungus gnats. For instance, the combined application of M. anispoliae and the predatory mite (Gaeolaelaps aculeifer) generated a better efficacy to control fungus gnat larvae L. auripilla (Tavoosi Ajvad et al., 2019). The control efficacy of the fungus gnat $B$. impatiens was enhanced by the combination of an EPN (Heterorhabditis indica) and a predatory mite (Stratiolaelaps scimitus) (Acharya et al., 2019).

In addition, the combined application of M. brunneum and the botanical compounds in four substrates with different constituents revealed that this combined formulation caused a higher reduction of adult emergence in all substrates, except the standard substrate. Growing medium type, based on the different components, might be regarded as a key factor for the development of fungus gnats. In this regard, Jagdale et al. (2004) indicated the substrate type and components might provide a favorable site for development and reproduction of fungus gnats. Additionally, substrates containing hardwood bark texture (Hoitink, 1989) and high level of microbial activity (Freeman, 1983) might lead to increased fungus gnat populations. In our study, Fruhstorfer Erde substrate was more attractive to fungus gnat larvae to finalize their life cycle to adults. Hence, Fruhstorfer Erde (Typ P) was selected as an optimal substrate for our further bioassays.

\section{Conclusion}

This is the first report that demonstrated the efficacy of the biopesticides ( $M$. brunneum or Bt) in combination with a botanical compound (Minx) against fungus gnat larvae. We found that $M$. brunneum in combination with Minx resulted in fewer numbers of emerging fungus gnat adults. The botanical compound Minx, although attractive to fungus gnat larvae, has an additive role on the effectiveness of $M$. brunneum. Therefore, this formulation might offer a new approach as an "attract and kill" strategy, resulting in a higher efficacy to suppress fungus gnat populations. Future works will focus on improving the effectiveness of the combined application of bioinsecticides and the botanical capsule Minx against fungus gnat larvae. We will also focus on the efficacy of this co-formulation on attraction and oviposition of the fungus gnat adults. 


\section{Acknowledgements}

We would like to thank Bianca Tappe and Marie Nörthemann for their technical assistance. We would like also to thank Jörg Rademacher (Katz Biotech AG, Baruth, Germany) for providing the botanical compounds. Furthermore, we would like to thank Maik Knobel for providing the different types of substrates.

\section{References}

Acharya, R., Hwang, H. S., Shim, J. K., Yu, Y. S. \& Lee, K. Y. (2019) Control efficacy of fungus gnat, Bradysia impatiens, enhanced by a combination of entomopathogenic nematodes and predatory mites. Biological Control, 138.

Andreadis, S. S., Cloonan, K. R., Bellicanta, G. S., Paley, K., Pecchia, J. \& Jenkins, N. E. (2016) Efficacy of Beauveria bassiana formulations against the fungus gnat Lycoriella ingenua. Biological Control, 103, 165-171.

Baker, K. D., Rice, J. S., Leemon, D. M. \& James, P. J. (2018) Horizontal transmission of Metarhizium anisopliae (Hypocreales: Clavicipitacea) and the effects of infection on oviposition rate in laboratory populations of Musca domestica (Diptera:Muscidae). Pest Management Science, 74, 987-991.

Cabrera, A. R., Cloyd, R. A. \& Zaborski, E. R. (2003) Effect of monitoring technique in determining the presence of fungus gnat, Bradysia spp. (Diptera: Sciaridae), larvae in growing medium. Journal of Agricultural and Urban Entomology, 20, 41-47.

Chambers, R. J., Wright, E. M. \& Lind, R. J. (1993) Biological control of glasshouse sciarid flies (Bradysia spp.) with the predatory mite, Hypoaspis miles, on cyclamen and poinsettia. Biocontrol Science and Technology, 3, 285-293.

Cloyd, R. A. \& Dickinson, A. (2006) Effect of Bacillus thuringiensis subsp. israelensis and neonicotinoid insecticides on the fungus gnat Bradysia sp nr. coprophila (Lintner) (Diptera: Sciaridae). Pest Management Science, 62, 171-177.

Cloyd, R. A., Dickinson, A., Larson, R. A. \& Marley, K. A. (2007a) Effect of growing media and their constituents on fungus gnat, Bradysia sp. nr. coprophila (Lintner) adults. Insect Science, 14, 467-475.

Cloyd, R. A. (2008) Management of fungus gnats (Bradysia spp.) in greenhouse and nurseries. Floriculture and Ornamental Biotechnology, 2, 84-89. 
Cloyd, R. A. (2015). Ecology of fungus gnats (Bradysia spp.) in greenhouse production systems associated with disease-interactions and alternative management strategies. Insects, 6, 325332.

El-Hamalawi, Z. A. \& Stangellini, M. E. (2005) Disease development on lisianthus following aerial transmission of Fusarium avenaceum by adult shore flies, fungus gnats, and moth flies. Plant Disease, 89, 619-623.

El-Sayed, A. M., Suckling, D. M., Byers, J. A., Jang, E. B. \& Wearing, C. H. (2009) Potential of ' lure and kill ' in long-term pest management and eradication of invasive species. Journal of Economic Entomology, 102, 815-835.

Freeman, P. (1983). Sciarid flies. In: Fitton, M. G. (eds.) Handbook for the identification of British insects. Royal entomological society of London, $68 \mathrm{pp}$.

Frouz, J., Naváková, A. \& Jones, T. H. (2002) The potential effect of high atmospheric CO2 on soil fungi-invertebrate interactions. Global Change Biology, 8, 339-344.

Gillespie, D. R. \& Menzies, J. G. (1993) Fungus gnats vector Fusarium oxysporum f. sp. radicislycopersici. Annals of Applied Biology, 123, 539-544.

Golo, P. S., Gardner, D. R., Grilley, M. M., Takemoto, J. Y., Krasnoff, S. B., Pires, M. S., Fernandes, E. K. K., Bittencourt, V. R. E. P. \& Roberts, D. W (2014) Production of destruxins from Metarhizium spp. fungi in artificial medium and in endophytically colonized cowpea plants. PLoS One, 9, e104946.

Guerenstein, P. G. \& Hildebrand, J. G. (2008) Roles and effects of environmental carbon dioxide in insect life. Annual Review of Entomology, 53, 161-178.

Hamlen, R. A. \& Mead, F. W. (1979) Fungus gnat larval control in greenhouse plant production. Journal of Economic Entomology, 72, 269-271.

Hanitzsch, M., Przyklenk, M., Witte, S., Nordzieke, B. H. \& Patel, A. (2015) Encapsulation of Bt toxin against fungus gnat larvae. INBIOSOIL Symposium, Göttingen.

Harris, M. A. (1995) Dissemination of the phytopathogen Thielaviopsis basicola by the fungus gnat Bradysia coprophila and biological control of these pests by Fusarium proliferatum and steinernematid nematodes. Ph.D. Thesis, University of Georgia, Athens, GA, USA.

Harris, M. A., Oetting, R. D. \& Gardner, W. A. (1995) Use of entomopathogenic nematodes and a new monitoring technique for control of fungus gnats, Bradysia coprophila (Diptera: Sciaridae), in floriculture. Biological control, 5, 412-418. 
Höfte, H. \& Whiteley, H. R. (1989) Insecticidal crystal proteins of Bacillus thuringiensis, Microbiological Reviews, 53, 242-255.

Hoitink, H. A. J. (1989) Integrated control of Pythium, Rhizoctonia and Fusarium diseases. In: Proceedings of the Fifth Conference on Insect and Disease, Society of American Florists, Alexandria, VA, 116-121.

Jagdale, G. B., Casey, M. L., Grewal, P. S. \& Lindquist, R. K. (2004) Application rate and timing, potting medium, and host plant effects on the efficacy of Steinernema feltiae against the fungus gnat, Bradysia coprophila, in floriculture. Biological Control, 29, 296-305.

James, R. L., Dumroese, R. K. \& Wenny, D. L. (1995) Botrytis cinerea carried by adult fungus gnats (Diptera: Sciaridae) in container nurseries. Tree Planters Notes, 46, 48-53.

Jarvis, W. R., Shipp, J. L. \& Gardiner, R. B. (1993) Transmission of Pythium aphanidermatum to greenhouse cucumber by the fungus gnat Bradysia impatiens (Diptera: Sciaridae). Annals of Applied Biology, 122, 23-29.

Jess, S. \& Kilpatrick, M. (2000) An integrated approach to the control of Lycoriella solani (Diptera: Sciaridae) during production of the cultivated mushroom (Agaricus bisporus). Pest Management Science, 56, 477-485.

Kalb, D. W. \& Millar, R. L. (1986) Dispersal of Verticillium albo atrum by the fungus gnat (Bradysia impatiens). Plant Disease, 70, 752-753.

Koller, M. (2011) Comparison of Steinernema feltiae, Bacillus thuringiensis israelensis and Azadirachtin to control Sciarids in Organic potted herbs. Acta Horticulturae (ISHS), 951, 179184.

Margalit, J., Lahkim-Tsror, L., Pascar-Gluzman, C. \& Bobroglo, H. (1985) Biocontrol of mosquitoes in Israel. In: Laird, M. \& Miles, J. W. (eds.) Integrated mosquito control methodologies. Academic Press, 361-374.

Meers, T. L. \& Cloyd, R. A. (2005) Egg-laying preference of female fungus gnat Bradysia sp. nr. coprophila (Diptera: Sciaridae) on three different soilless substrates. Journal of Economic Entomology, 98, 1937-1942.

Molloy, D., Gaugler, R. \& Jamnback, H. (1981) Factors influencing efficacy of Bacillus thuringiensis var. israelensis as a biological control agent of black fly larvae. Journal of Economic Entomology, 74, 61-64.

Ohana, B., Margalit, J. \& Barak, Z. (1987) Fate of Bacillus thuringiensis subsp. israelensis under simulated field conditions. Applied and Environmental Microbiology, 53, 828-831. 
Osborne, L. S., Boucias, D. G. \& Lindquist, R. K. (1985) Activity of Bacillus thuringiensis var. israelensis on Bradysia coprophila (Diptera: Sciaridae). Journal of Economic Entomology, 78, 922-925.

Paula, A. R., Silva, L. E. I., Ribeiro, A., Butt, T. M., Silva, C. P. \& Samuels, R. I. (2018) Improving the delivery and efficiency of fungus-impregnated cloths for control of adult Aedes aegypti using a synthetic attractive lure. Parasites \& Vectors, 11, 285-294.

Quesada-Moraga, E., Carrasco-Dı'az, J. A. \& Santiago-A' Ivarez, C. (2006) Insecticidal and antifeedant activities of proteins secreted by entomopathogenic fungi against Spodoptera littoralis (Lep., Noctuidae). Journal of Applied Entomology, 130, 442-452.

Quiot, J. M., Vey, A. \& Vago, C. (1985) Effects of mycotoxins on invertebrate cells in vitro. Advances in Cell Culture, 4, 199-212.

Quintela, E. D. \& McCoy, C. W. (1998) Synergistic effect of imidacloprid and two Entomopathogenic fungi on the behavior and survival of larvae of Diaprepes abbreviatus (Coleoptera: Curculionidae) in soil. Journal of Economic Entomology, 91, 110-122.

Samuels, R. I., Reynolds, S. E. \& Charnley, A. K. (1988) Calcium channel activation of insect muscle by destruxins, insecticidal compounds produced by the entomopathogenic fungus Metarhizium anisopliae. Comparative Biochemistry and Physiology, 90C, 403-412.

Shamshad, A., Clift, A. D. \& Mansfield, S. (2008) Toxicity of six commercially formulated insecticides and biopesticides to third instar larvae of mushroom sciarid, Lycoriella ingenua Dufour (Diptera: Sciaridae), in New SouthWales, Australia. Australian Journal of Entomology, $47,256-260$.

Smith, T. M. \& Stratton, G. W. (1986) Effects of synthetic pyrethroid insecticides on nontarget organisms. Residue Reviews, 97, 93-120.

Tavoosi Ajvad, F., Madadi, H., Michaud, J. P., Zafari, D. \& Khanjani, M. (2019) Combined applications of an entomopathogenic fungus and a predatory Mite to control fungus gnats (Diptera: Sciaridae) in mushroom production. Biological Control, 141.

Vega, F. E., Posada, F., Aime, M. C., Pava-Ripoll, M., Infante, F. \& Rehner, S. A. (2008) Entomopathogenic fungal endophytes. Biological Control, 46, 72-82.

Wilkinson, J. D. \& Daugherty, D. M. (1970a) The biology and immature stages of Bradysia impatiens (Diptera: Sciaridae). Annals of the Entomological Society of America, 63, 656-660. 
Chapter 3

An attract and kill formulation for fungus gnat control with combining a biopesticide and a botanical compound

Mahsa Dehghani", Jörg Rademacher and Stefan Vidal

* Corresponding author 


\title{
An attract and kill formulation for fungus gnat control with combining a biopesticide and a botanical compound
}

M. Dehghani ${ }^{1 *}$, J. Rademacher ${ }^{2}$ and S. Vidal ${ }^{1}$

${ }^{1}$ Georg-August-Universität Göttingen, Department of Crop Sciences, Agricultural Entomology, Grisebachstrasse 6, 37077 Göttingen, Germany

${ }^{2}$ Katz Biotech AG, Baruth, Germany

*Corresponding author: E-mail address: mdehgha@gwdg.de

\begin{abstract}
Fungus gnats of the genus Bradysia spp. (Diptera: Sciaridae) are one of the most common pests in greenhouse cultures. Organic production systems need to fall back on biological control strategies for fungus gnat control. In a first series of bioassays, we studied the efficacy of combining a biopesticide and a botanical compound in an "attract and kill" strategy targeting larval fungus gnat. The entomopathogenic fungus isolate Metarhizium brunneum ( $\mathrm{Cb} 15)$ and two biopesticides (Neem Azal and Spinosad), when applied alone, proofed to be effective against fungus gnat larvae. Additionally, the botanical capsules (Minx) indicated an attractant effect for the fungus gnat larvae. Therefore, the efficacy of $M$. brunneum or two biopesticides in combination with the botanical compound against fungus gnat larvae was assessed. Significantly fewer adult numbers emerged from the combined treatments, specifically Spinosad in combination with the botanical compound ( $2 \%)$, as compared to the other treatments or the control (42\%), pointing to an additive effect of combining the two agents. In the next series of bioassays, the egg-laying behavior of females when exposed to the isolate of $M$. brunneum and biopesticides (Bacillus thuringiensis, Bt or Spinosad) were evaluated. Fungus gnats preferred to lay more eggs in Spinosad treatment as compared to the other treatments or the control. However, the eggs laid by the females in Spinosad treatments could not successfully develop to adults, resulting in an efficient reduction of adult emergence (99\%). These results offer an "attract and kill" approach, which could help to efficiently control fungus gnat populations.
\end{abstract}

Keywords: fungus gnats, Metarhizium brunneum, Spinosad, attract and kill, egg-laying behavior 


\section{Introduction}

The high humidity of greenhouses provide a suitable condition for reproduction of a number of insects such as fungus gnat species (i.e. Bradysia spp.; Diptera: Sciaridae) (Stanton \& Sanders, 1998). Fungus gnats of the genus Bradysia spp. are one of the most common insect pests in greenhouses (Harris et al., 1995). This genus includes various species, but the most common species as crop pests include B. impatiens and B. coprophila (Cloyd, 2008). The larval stage of fungus gnats feed primarily on organic material and fungi in the soil (Kennedy, 1974), but they can cause serious plant damage by feeding on the healthy roots and stems, vectoring fungal pathogens such as Fusarium spp., Pythium spp. and Verticillium spp., and predisposing plants to fungal attack (Gillespie \& Menzies, 1993, El-Hamalawi, 2008, Braun et al., 2012). Hence, it is important for greenhouse producers to control fungus gnat larvae to avoid economic losses (Leath \& Newton, 1969, Hamlen \& Mead, 1979). Fungus gnat adults cause minimal plant damage (Cloyd, 2000), but they are able to disseminate some fungal spores (e.g. Botrytis cinerea) from infected to healthy plants (James at al., 1995). Adults tend to fly near the surface of the substrate and females lay eggs 100 to 200 in the crevices of the substrate (Cloyd, 2008).

The overlapping generations of fungus gnats in the greenhouse has led to the excessive use of synthetic insecticides, which caused limitations, such as insect resistance, pollution in the environment and human health concerns (Jelinek \& Azzopardi, 2010, Bethke \& Dreistadt, 2013). Hence, finding effective and environmentally friendly methods is necessary for controlling fungus gnats in greenhouses. An alternative strategy which should be considered to control fungus gnats is the use of biological control agents (BCAs) including a predatory mite species (Wright \& Chambers, 1994), a rove beetle (Carney et al., 2002), and entomopathogenic nematodes (EPN) (Harris et al., 1995, Dreistadt, 2001, Jagdale et al., 2004), the natural insecticide Bacillus thuringiensis (Bt) (Osborne at al., 1985, Cloyd, 2000, Cloyd \& Dickinson, 2006) or the botanical Neem (Cloyd, 2008) respectively.

Entomopathogenic fungi (EPFs) are being promoted and evaluated as inundative biological control agents of insect pests, mites and ticks (Butt et. al., 2001, Vincet et al., 2007). Metarhizium anisopliae (Metschnikoff) (Hypocreals: Clavicipitaceae) is one of the most commercially used EPF that has been reported as a natural antagonist for a various species of Diptera, e.g. mosquitos (Culicidae) (Paula et al., 2018, Popko et al., 2018), house flies (Baker et al., 2018), Drosophila suzukii (Drosophilidae) (Woltz et al., 2015). M. anisopliae has also been reported as a biological control agent of Lycoriella auripilla (Diptera: Sciaridae) larvae (Tavoosi Ajvad et al., 2019). 
Metarhizium spp. produce toxins such as destruxin, which can be highly toxic to insects by injection, ingestion and topical application (Poprawski et al., 1994, Golo et al., 2014). In recent years, the application of EPFs for pest control achieved increasing attention by researcher and enterprises, due to the cost effective mass production of propagules, high efficacy of fungal strains to infect insects and the reasonable costs (Vega et al., 2008).

Biological control offers several effective and low risk natural pesticides, which can be used to manage fungus gnats in greenhouses and nurseries. For instance, the natural insecticide Neem, with its active ingredient azadirachtin, has been shown to be effective against fungus gnat larvae (Mordue \& Nisbet, 2000, Cloyd, 2008). In addition, Ludwig \& Oetting (2001) demonstrated a high efficiency of azadirachtin in reducing adult emergence of B. coprophila. Koller (2011) demonstrated the efficacy of azadirachtin against fungus gnat Bradysia spp.

Another bioinsecticide, Spinosad is the active ingredient in the insecticide, Spintor ${ }^{\circledR}$. Spinosad is derived from the species of Actinobacteria Saccharopolyspora spinosa (Mertz \& Yao, 1990) that during fermentation generates two biological active compounds, spinosyns $A$ and $D$. These are responsible for the insecticidal properties (Kirst et al., 1992, Thompson et al., 1997). This natural insecticide has a low toxicity to mammals and a safe environmental property (Cleveland et al., 2001, Dow Agrosciences, 2001); hence, it has been classified as a bioinsecticide (Copping \& Menn, 2000). Salgado (1998) has demonstrated the mode of action of Spinosad including excitation of the insect nerve system, resulting in paralysis and death. Spinosad has been shown to be effective for the control of Diptera, e.g. Megaselia halterata (Phoridae) (Erler et al., 2009), cherry fruit fly, Rhagoletis indifferens (Tephritidae) (Yee, 2018) and eye gnat Liohippelates collusor (Chloropidae) (Jiang \& Mulla, 2006). The effectiveness of this bioinsecticide needs to be investigated to develop a more efficient management program for controlling fungus gnats.

Performance of the bioinsecticides can be enhanced by an effective formulation, resulting in higher efficacy and exposure of insects to the killing agent (Lacey et al., 2001, Jackson et al., 2010). For instance, an additive effect has been already reported for the combined application of an entomopathogenic nematode (EPN) Heterorhabditis indica and a predatory mite Stratiolaelaps scimitus against fungus gnat larvae (B. impatiens) (Acharya et al., 2019). Tavoosi Ajvad et al. (2019) also reported that the EPF M. anisopliae in combination with a predatory mite (Gaeolaelaps aculeifer) increased fungus gnat (L. auripilla) mortality in mushroom production. Addditionally, a study by $\mathrm{Wu}$ et al. (2017) indicated a synergistic effect of the EPN Steinernema feltiae and thiamethoxam for the control of the fungus gnat species $B$. odoriphaga. 
We hypothesized an increased control efficiency of fungus gnats of the species $B$. impatiens by a combination of an attractive agent with a biopesticide in an "attract-and-kill" strategy. ElSayed et al. (2009) indicated that using this approach an insect pest can be attracted towards an attractive agent either via semiochemical signals, visual and acoustic signals or combinations of these cues, and might be thus more subjected to a killing agent. In this study, a botanical capsule (Minx) was applied as an attractant for fungus gnat larvae as described in a previous study. This capsule, containing mint oil was formulated in sodium ( $\mathrm{Na}$ )-alginate as a carrier material and stabilized by calcium alginate matrix as hydrogel.

The aims of this study were: (1) to evaluate whether the combination of a biopesticide (Metarhizium brunneum $\mathrm{Cb}$ 15, Neem Azal or Spinosad) and a botanical capsule improve the control efficiency of B. impatiens; (2) to select the most effective combined formulations for further experiments and (3) to determine whether fungus gnat adults are attracted to these combined formulations for oviposition.

\section{Materials and methods}

Insect rearing

A laboratory colony of the fungus gnat species $B$. impatiens was established with adults collected from Agricultural Entomology Section, Crop Science Department, Georg-August Universität Göttingen, Germany. Adults were reared in plastic pots $(11 \mathrm{~cm} \times 11 \mathrm{~cm} \times 12 \mathrm{~cm})$ filled with $350 \mathrm{gr}$ of a favorable growing medium containing $50 \% \mathrm{v} / \mathrm{v}$ medium milled white peat, $30 \% \mathrm{v} / \mathrm{v}$ frozenthrough black peat, $20 \% \mathrm{v} / \mathrm{v}$ green waste compost, $2 \mathrm{~kg}$ lime $/ \mathrm{m}^{3}, 1 \mathrm{~kg}$ Radigen $/ \mathrm{m}^{3}$ and $6 \mathrm{~kg}$ horn shives / $\mathrm{m}^{3}$ (Klasmann-Deilmann $\mathrm{GmbH}$; Geeste, Germany) supplemented with oatmeal (3 gr) and a shredded potato (Cloyd \& Zaborski, 2004). Fungus gnat's rearing were maintained at $23 \pm 2{ }^{\circ} \mathrm{C}$ and 16:8 $\mathrm{h}$ (L:D) photoperiod. To provide even-aged third-instar fungus gnat larvae for the experiments, adults were collected from the permanent colonies and released into a small plastic containers containing moist growing medium as described for rearing fungus gnats (KlasmannDeilmann $\mathrm{GmbH}$; Geeste, Germany), supplemented with oatmeal and potato for oviposition. $48 \mathrm{~h}$ after oviposition, these plastic containers were maintained in a room at $23 \pm 2{ }^{\circ} \mathrm{C}$ until third instar larvae emerged. Additional water was added to the substrate as needed. The newly hatched third instar larvae were collected with a cooked potato disk placed on the surface of the substrate. Larvae were isolated via a floating technique. The selected larvae were transferred to bioassay plastic cups using a fine brush (Cabrera et al., 2003). 


\section{Treatment preparation and application}

"Fungal preparation": The fungal strain used for this study, M. brunneum ( $\mathrm{Cb} 15)$, has been obtained from a culture maintained at the Agricultural Entomology section, Georg-August Universität Göttingen, Germany. This fungal strain was grown on potato extract glucose agar (PDA) (Carl Roth GmbH \& Co. KG, Karlsruhe, Germany) for ca. 3 weeks and maintained in a climate chamber (Biologischer Klimaschrank WB 750, Mytron Bio- und Solartechnik GmbH, Heilbad Heiligenstadt, Germany) at $25 \pm 1^{\circ} \mathrm{C}$ and $60 \pm 5 \% \mathrm{RH}$ in complete darkness. The conidial suspensions were prepared by scraping the surface of the Petri plate into a $50 \mathrm{ml}$ sterile demineralized water plus Tween 80 (0.1 \% v/v; Carl Roth ${ }^{\oplus}$, Germany). This initial conidial suspension was filtered through a sterilized fine mesh to remove mycelium and agar residues. The spore suspension was verified with light microscopy (200X magnification, BH2-HLSH, Olympus Corporation, Tokyo, Japan). The pure fungal suspension was adjusted by diluting the conidia with demineralized water to a final concentration of $1 \times 10^{7}$ spores $/ \mathrm{ml}$. The number of spores was estimated using a Neubauer Hemocytometer (0.1 mm depth) (Thoma CE, Paul Marienfeld GmbH \& Co.KG, Lauda-Königshofen, Germany).

"Attract": The botanical compounds were obtained from Katz Biotech AG; Baruth, Germany and stored in a cooling chamber $\left(6^{\circ} \mathrm{C}\right)$ until use. The composition of the botanical capsule (Minx) was made up of vegetable oil (approximately 50\% dry matter), ( $\mathrm{Na}$ )-alginate solution, silica gel, mint oil (different rates: $0.1 \%, 1 \%$ and $10 \%$ ) and water (5\% in dry matter). These components were suspended in a Na-alginate solution and this suspension was dripped with a syringe through droplet capillaries into a $\mathrm{CaCl}_{2}$ solution, where the droplets was solidified by a gelation and formed a hydrogel. The solid alginate capsule was dried in a fluid bed. The interspaces were filled with

mint oil. A solid form of this structure was used in this study (J. Rademacher, personal communication, Katz Biotech AG; Baruth, Germany). The Minx capsule preparation can be easily manufactured at a large scale.

\section{Experimental design}

\section{Experiment 1}

This experiment was conducted to determine the ovipositional preference of fungus gnats for $M$. brunneum ( $\mathrm{Cb} 15)$ individually or in combination with the botanical capsules Minx on water agar plates. In this trial, each Petri plate with $9 \mathrm{~cm}$ diameter (Sarstedt AG \& Co. KG, Nümbrecht, Germany) was filled with water agar (Agar-Agar, Kobe I, Carl Roth GmbH \& Co. KG, Karlsruhe, 
Germany). 2 g substrate (Fruhstorfer Erde Typ P 25; Hawita Gruppe GmbH, Vechta, Germany) supplemented with three to four pieces of oatmeal was placed on the surface of the water agar plate to provide a suitable site for fungus gnat females to lay eggs. $2 \mathrm{ml}$ of $\mathrm{M}$. brunneum (Cb 15) of the spore suspensions were applied directly at a rate of $1 \times 10^{7} \mathrm{spore} / \mathrm{ml}$ to the substrate or to the Minx capsule containing $0.1 \%$ mint oil buried in the substrate. Sterilized water was applied to the control substrates. The water agar plates were kept in a rearing tent (BugDorm-2 Insect Tents, MegaView Science Co.Ltd.; Taichung, Taiwan) ( $\mathrm{N}=2$ Petri plates / per treatment in each tent) at room temperature. Immediately after treatment, fifty fungus gnat adults (mixture of females and males; sex ratio about 50\%) were released into each experimental arena consisting of the water agar plates. This experiment was set up in a room at $23 \pm 2{ }^{\circ} \mathrm{C} .48 \mathrm{~h}$ after the release of the fungus gnat adults, the numbers of eggs laid by the females were recorded under a binocular microscope (ZEISS, Carl Zeiss Microscopy GmbH, Jena, Germany). This experiment was replicated six times, totally 12 Petri plates per treatment.

\section{Experiment 2}

The efficacy of Spinosad individually or in combination with the botanical capsules Minx was determined on attraction and oviposition of the fungus gnats. Each plastic cup was filled with 250 ml substrate (Fruhstorfer Erde Typ P 25; Hawita Gruppe GmbH, Vechta, Germany). $10 \mathrm{ml}$ of Spinosad suspensions were added at a rate of $1 \%$ (Spintor $^{\circledast}: 480 \mathrm{~g}$ Spinosad active ingredient per liter, Dow AgroSciences Kings Lynn Norfolk, United Kingdom) to the substrate treated with the botanical microcapsules Minx containing $0.1 \%$ mint oil. Treatments were mixed within substrate. After preparing the treatments, fifty fungus gnat adults (mixture of females and males; sex ratio about 50\%) were released into each experimental chamber. This trial was set up at the same condition as described in experiment $1.48 \mathrm{~h}$ post treatment (48 HPT), the adults were collected from the experimental chamber and the numbers of eggs laid by the females were counted under a binocular microscope (ZEISS, Carl Zeiss Microscopy GmbH, Jena, Germany). Each treatment was performed with six replicates ( $N=6$ experimental cups / treatment).

\section{Experiment 3}

In this experiment, the egg-laying behavior of fungus gnats when exposed to M. brunneum and two biopesticides (Bacillus thuringiensis, Bt or Spinosad) individually or in combination with the botanical capsules was determined. This bioassay was conducted in the experimental chamber 
with six replicates consisting of a plastic cup containing $250 \mathrm{ml}$ substrate (Fruhstorfer Erde Typ P 25; Hawita Gruppe GmbH, Vechta, Germany) treated individually with the M. brunneum spore suspensions and two biopesticides. In each experimental cup, the surface of the substrate was covered using a fine mesh with $9 \mathrm{~cm}$ diameter. Prior to treating the substrate, a small portion of the substrate $(2 \mathrm{~g})$ and three to four pieces of oatmeal were added to cover the mesh to provide an appropriate site for fungus gnat adults to lay eggs. This mesh assisted in counting the numbers of eggs laid by the females. $10 \mathrm{ml}$ of the M. brunneum spore suspensions or two biopesticides (Bt or Spinosad) were drench-applied directly to the substrate on the mesh treated with the botanical microcapsule containing $0.1 \%$ mint oil. The biopesticides used in this study were: M. brunneum (1 $\times 10^{7}$ spore $/ \mathrm{ml}$ ), the recommended rate of Bt BioMükk ${ }^{\circledR}$ WDG $300-400 \mathrm{~g} / 100 \mathrm{~m}^{2}$ in $1 \mathrm{~L} / 1$ $\mathrm{m}^{2}$ water (Biofa; Münsingen, Germany) (0.032 g / $78.5 \mathrm{~cm}^{2}$ in $7.85 \mathrm{ml}$ water per cup), and Spintor (10 $\mathrm{ml}$ Spinosad suspension at a rate of $1 \%$ per cup). Control cups were treated with $10 \mathrm{ml}$ of water. Fifty fungus gnat adults were aspirated into each experimental chamber containing the treated cups and allowed to oviposit freely. $48 \mathrm{~h}$ after the release of fungus gnats, the adults were removed from the chamber using an aspirator and the numbers of eggs laid by the females on the mesh were counted. After the examination, the eggs were returned into the plastic cups. Cups were incubated in a growth chamber at $25 \pm 1^{\circ} \mathrm{C}$ and $65 \pm 5 \% \mathrm{RH}$ with a photoperiod: 16:8 $\mathrm{h}$ (Biologischer Klimaschrank WB 750, Mytron Bio- und Solartechnik GmbH, Heilbad Heiligenstadt, Germany). 22 days after treatment, the total numbers of emerging adults in each cup using a yellow sticky card fixed at the top to trap the emerging adults were counted. Each treatment was performed with six replicates ( $N=6$ experimental cups / treatment).

\section{Experiment 4}

The efficacy of the Minx microcapsules at different concentrations of mint oil $(0.1 \%, 1 \%$ and $10 \%)$ was evaluated on fungus gnat larvae. A transparent plastic cup (300 ml, Nette Papier GmbH, Göttingen, Germany) was filled with 250 ml substrate (Fruhstorfer Erde Typ P 25; Hawita Gruppe $\mathrm{GmbH}$, Vechta, Germany). Minx microcapsules at different rates $(10,50,100$ and $150 \mathrm{mg})$ for each concentration were added individually to the $10 \mathrm{ml}$ of fungal suspensions of $\mathrm{M}$. brunneum (Cb 15) at a rate of $1 \times 10^{7} \mathrm{spore} / \mathrm{ml}$ and then mixed with the fungal suspensions. The treated capsules were then transferred to the substrate. Moreover, the additional fungal suspensions of $M$. brunneum were added to the substrates and the treatments were mixed within substrate. It is notably that some capsules treated with the fungal suspensions were transferred individually to 
plates of PDA (potato extract glucose agar) (Carl Roth GmbH \& Co. KG, Karlsruhe, Germany) and incubated in a climatic chamber $\left(25 \pm 1^{\circ} \mathrm{C}\right)$ for ca. 3 weeks to evaluate whether the capsules have been coated with the spore suspensions. The resulting colonies were identified microscopically by spores. However, the exact rate of conidia that was attached to the capsule was not determined. 24h post treatment (24 HPT), 20 newly moulted third instar larvae were released into each experimental cup using a fine brush. These plastic cups were stored in a climatic chamber at $25 \pm$ $1^{\circ} \mathrm{C}$ and $65 \pm 5 \% \mathrm{RH}$ with a photoperiod: 16:8 h (Biologischer Klimaschrank WB 750, Mytron Biound Solartechnik GmbH, Heilbad Heiligenstadt, Germany) to complete their life cycle to adults. Each cup was closed with a lid. Adult emergence was monitored during a period of two weeks using a yellow sticky card $(2.5 \times 2.5 \mathrm{~cm})$ attached to the top of each plastic cup, which effectively captured the fungal gnat adults. Each treatment was replicated 10 times ( $N=10$ experimental cups / treatment). For the control treatment, $10 \mathrm{ml}$ sterilized water was applied to the substrate.

\section{Experiment 5}

In this laboratory bioassay, the efficacy of two different rates of $M$. brunneum ( $\mathrm{Cb} 15)$ individually or in combination with the botanical compound Minx was assessed to control fungus gnat larvae. This experiment was similar to experiment 4, except that $10 \mathrm{ml}$ of the M. brunneum spore suspensions were added at a rate of $1 \times 10^{7} \mathrm{spore} / \mathrm{ml}$ and $1 \times 10^{9} \mathrm{spore} / \mathrm{ml}$ individually to the substrate (Fruhstorfer Erde Typ P 25; Hawita Gruppe GmbH, Vechta, Germany) treated with the botanical capsules Minx. These microcapsules containing $0.1 \%$ and $10 \%$ mint oil were applied at two different rates $(2 \mathrm{mg}$ and $10 \mathrm{mg}$ ) to the substrate. These treatments were mixed within the substrate. $24 \mathrm{~h}$ after setting up these treatments (HPT), twenty third instar fungus gnat larvae were released into each experimental cup and incubated in a climatic chamber as described in experiment 4. Control cups received $10 \mathrm{ml}$ sterilized water. After two weeks, the total numbers of trapped adults in each cup was counted inspecting the yellow sticky card. In this experiment, ten replications per treatment were used.

\section{Experiment 6}

This bioassay was conducted to determine the effectiveness of $M$. brunneum $(\mathrm{Cb} 15)$ individually or in combination with the lowest rate of the Minx capsules ( $2 \mathrm{mg}$ ) against fungus gnat larvae. In this experiment, the set up and procedure was the same as described in the $4^{\text {th }}$ trial. The botanical capsules containing $0.1 \%$ mint oil at a rate of $2 \mathrm{mg}$ were added to $10 \mathrm{ml}$ of the fungal suspensions 
of $M$. brunneum $(\mathrm{Cb} 15)$ at a rate of $1 \times 10^{7} \mathrm{spore} / \mathrm{ml}$ and then mixed with the fungal suspensions. The treated capsules were applied directly to the substrate. The additional fungal suspensions (10 $\mathrm{ml}$ ) were then added to the substrates and the treatments were combined within substrate (Fruhstorfer Erde Typ P 25; Hawita Gruppe GmbH, Vechta, Germany). 24 after treatment, twenty third instar larvae were released into each experimental cup as described above and allowed to develop to adults. After two weeks, the amount of the emerging adults from each experimental cup was monitored using yellow sticky cards ( $N=10$ experimental cups / treatment). Control substrates were treated with sterilized water with the same volume as the fungal treatment. However, the exact rate of conidia that was attached to the botanical capsules was not determined.

\section{Experiment 7}

The efficacy of $M$. brunneum and two biopesticides (Neem Azal and Spinosad) individually or in combination with the botanical compound Minx for fungus gnats control was tested. In this laboratory bioassay, the botanical capsule Minx containing $0.1 \%$ mint oil at the rate of $2 \mathrm{mg}$ was firstly added to the substrate (Fruhstorfer Erde Typ P 25; Hawita Gruppe GmbH, Vechta, Germany). $10 \mathrm{ml}$ of the M. brunneum (Cb 15) spore suspensions and biopesticides (Neem Azal ${ }^{\circledR}$ and Spintor $\left.{ }^{\oplus}\right)$ were drench-applied at either the same rate mentioned above $\left(1 \times 10^{7} \mathrm{spore} / \mathrm{ml}\right)$ or the recommended application rate [(Neem Azal $^{\oplus}: 1 \%$ azadirachtin active ingredient obtained from Trifolio-M GmbH, Germany) and Spintor ${ }^{\oplus}$ : 480g Spinosad active ingredient per liter obtained from Dow AgroSciences Kings Lynn Norfolk, United Kingdom)] to the substrate treated with the botanical microcapsules Minx. The concentration of Spinosad was based on preliminary doseresponse bioassays using third instar fungus gnat larvae with a concentration of $1 \%$ as the minimum concentration was chosen for our experiments (unpublished results). Control substrate received $10 \mathrm{ml}$ of the sterilized water. $24 \mathrm{~h}$ post treatment, twenty newly hatched third instar larvae were transferred into each experimental cup and maintained in the growth chamber (air temperature and humidity: $25 \pm 1^{\circ} \mathrm{C}, 65 \pm 5 \%$; photoperiod: 16:8 h). Each cup was closed with a lid. The emergence of adults was recorded via a yellow sticky trap attached at the top of each plastic cup during a period of two weeks. Each treatment was performed with 10 replicates $(\mathrm{N}=10$ experimental cups / treatment), totaling 200 larvae per treatment. 


\section{Data analysis}

All statistical tests were analyzed with $\mathrm{R}$ version 3.5.3. All data were first checked for normality and error variance of homoscedasticity. In the screening experiments, a general linear model (GLM) with quasipoisson distribution was used to analyze the adult emergence obtained from the efficacy of a biopesticide individually or in combination with the botanical compound to control fungus gnat larvae (experiments 4, 6 and 7). Additionally, the emergence of fungus gnat adults (independent variable) in experiment 5 and the number of eggs laid by the females (independent variable) in experiments 2 and 3 were analyzed using one-way ANOVA. The emergence rate of fungus gnat adults in experiment 3 was also analyzed using GLM with quasipoisson distribution. In experiment 1, the egg-laying behavior of fungus gnats when exposed to $M$. brunneum alone or in combination with the botanical capsule on water agar plates was analyzed using GLM with quasipoisson distribution. Significant differences between treatments were determined using the Tukey Honestly test at $p<0.05$.

\section{Results}

\section{Experiment 1}

The egg-laying behavior of fungus gnat females indicated that the females laid significantly more eggs in botanical capsules-treated plates (36.22 \pm 12.85 ; mean $\pm \mathrm{SE}$ ) as compared to the fungal or the control treatments $\left(F_{3,44}=2.54, p<0.05\right.$; Fig. 1). Furthermore, there were no significant differences among $M$. brunneum alone $(13.66 \pm 3.42$; mean $\pm \mathrm{SE})$ or in combination with the botanical capsules Minx $(11 \pm 5.40$; mean $\pm S E)$ and the control $(13.16 \pm 5.58$; mean $\pm S E)$ in terms of the number of eggs laid; However, there were no significant differences between fungal and control treatments $\left(F_{2,33}=0.08, p=0.92\right)$. 


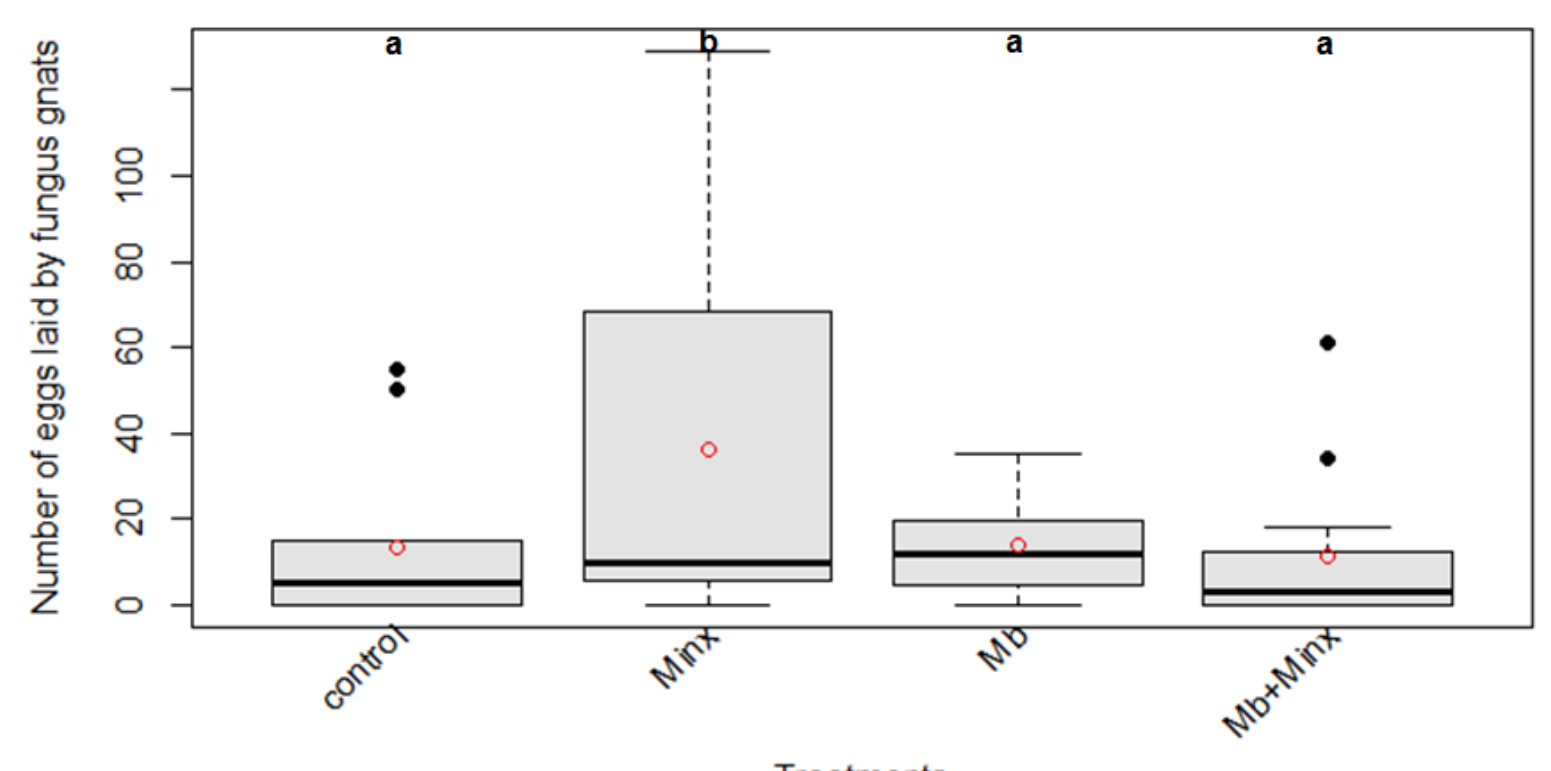

Treatments

Fig. 1: Number of eggs laid by the females in different treatments on water agar plates. Control, Minx: Botanical capsule containing mint oil; Mb: M. brunneum; Mb + Minx: Combining $M$. brunneum and botanical capsule. Fifty fungus gnat adults were released into each experimental chamber ( $N=2$ petri plates / treatment in each chamber; Six experimental chambers). Different letters indicate significant differences between treatments following Tukey HSD test $\left(F_{3,44}=2.54, p\right.$ $<0.05)$.

\section{Experiment 2}

There were no significant differences among the treatments tested in this trial in terms of numbers of eggs laid. Fungus gnat females preferred to lay more eggs, although not significantly, in Spinosad-treated cups individually or in combination with the botanical compound (65.16 \pm 13.62 and $64.16 \pm 14.67$; mean $\pm \mathrm{SE}$ ) as compared to the botanical compound or the control treatments $\left(F_{3,20}=0.65, p=0.60 ;\right.$ Fig. 2). Additionally, the number of eggs laid by the females in the substrates containing the botanical capsule Minx $(61.5 \pm 13.81$; mean \pm SE) were higher than the control substrate with water only $(41.16 \pm 13.94$; mean $\pm \mathrm{SE})$, although there were no significant differences between the botanical capsules and the control in terms of number of eggs laid $\left(F_{1,10}=1.07, p=0.32\right)$. 


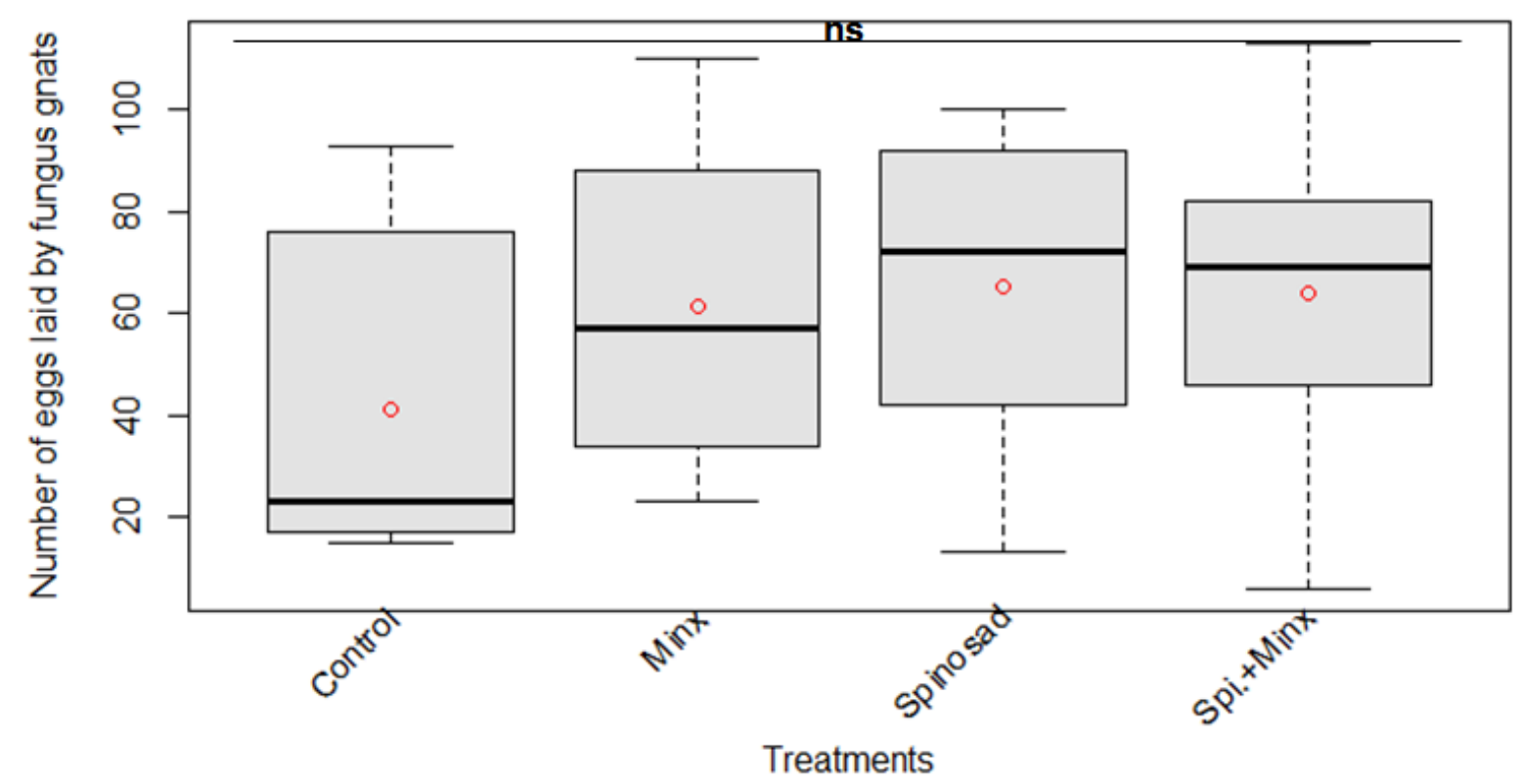

Fig. 2: Number of eggs laid by female fungus gnats in different treatments including Control, Minx: Botanical capsule containing mint oil, Spinosad, Spi. + Minx: Combining Spinosad and botanical capsule. ns indicates no significant differences between treatments as determined by Tukey HSD test $\left(F_{3,20}=0.65, p=0.60\right)$.

\section{Experiment 3}

Fungus gnat females tended to lay more eggs, although not significantly, in Spinosad-treated cups individually or in combination with the botanical capsule $(232.5 \pm 45.7$; mean \pm SE and $264.6 \pm$ 62.6; mean $\pm \mathrm{SE})$, respectively, as compared to the fungal or $\mathrm{Bt}$ treatments or the control $\left(\mathrm{F}_{7,40}=\right.$ 2.21, $p=0.054$; Fig. 3). Additionally, a higher number of eggs were laid by fungus gnats in the substrate containing the botanical capsules $(184.50 \pm 26.14$; mean $\pm \mathrm{SE}$ ) in comparison to the control substrate with water only $(124.8 \pm 19.86$, mean $\pm S E)$, although there were no significant differences between the botanical compound Minx and the control in terms of number of eggs laid. Furthermore, the mean number of eggs laid by females in the substrates treated with $M$. brunneum and $\mathrm{Bt}$ individually or in combination with the botanical capsules ranged from $133 \pm$ 25.06 (mean \pm SE) for Bt to $193.5 \pm 19.88$ (mean \pm SE) for M. brunneum in combination with the botanical capsule $(=M b+M i n x)$, with no significant differences between the treatments $\left(F_{3,20}=\right.$ 1.27, $\mathrm{p}=0.31)$. In addition to data on the egg-laying behavior, the emergence of adults from $M$. brunneum and two biopesticides individually or in combination with the botanical compound was 
evaluated. The data collected from this trial demonstrated that fewer adults hatched from eggs laid by females in the substrates treated with $M$. brunneum and two biopesticides (Bt and Spinosad) individually or in combination with the botanical capsules as compared to the control treatments $\left(F_{7,40}=9.09, p<0.001 ; F i g\right.$. 4). The combination of these treatments with the botanical compounds resulted in an additive effect as shown by the reduced number of adults emerging, although not significantly, compared to the single fungal or biopesticide treatments. Specifically, the eggs laid by females in Spinosad-treated cups could not successfully develop to adults. Hence, the mean number of adult emergence significantly decreased in Spinosad treatments when applied alone (99.8\%) or in combination with the botanical capsules (99.9\%) as compared to the other treatments $\left(F_{5,30}=8.35, p<0.001\right)$. In addition, the percentage of adult emergence from eggs laid in M. brunneum and Bt treatments varied from $2.93 \%$ for M. brunneum in combination with Minx capsules ( $=M b+\operatorname{Minx}$ ) to $5.7 \%$ for the Bt treatment in combination with Minx capsules $(=B t+\operatorname{Min} x)$.

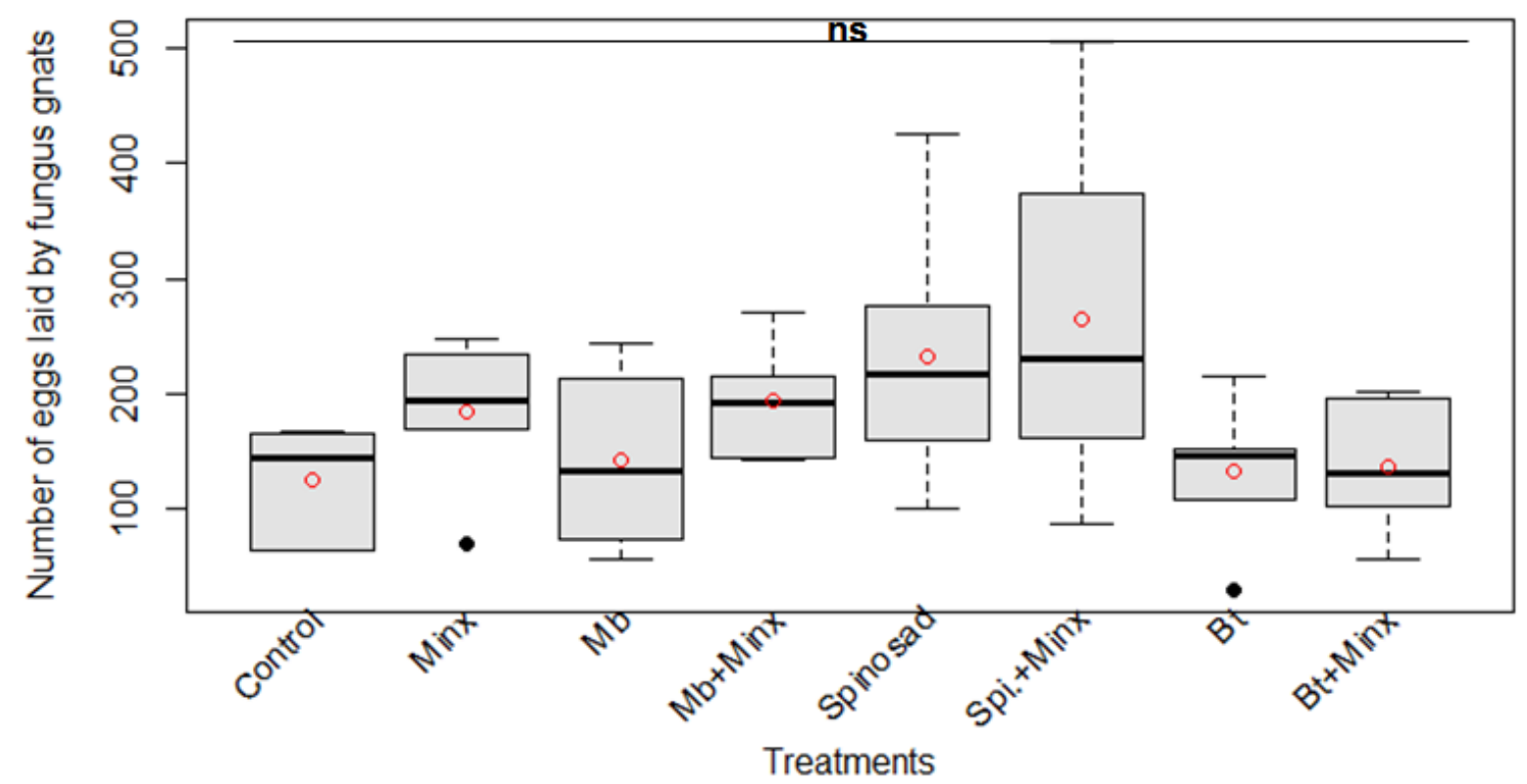

Fig. 3: Number of eggs laid by female fungus gnats when exposed to M. brunneum and two biopesticides (Bt and Spinosad) alone or in combination with the botanical compound Minx. Minx: Botanical capsule containing mint oil; Mb: M. brunneum; Mb + Minx: Combining M. brunneum and botanical capsule; Spi. + Minx: Combining Spinosad and botanical capsule; Bt: Bacillus thuringiensis; Bt + Minx: Combining Bt and botanical capsule. Fifty fungus gnats were released into each experimental chamber ( $N=6$ experimental cups / treatment). $\mathbf{n s}$ indicates no significant differences between treatments following Tukey HSD test $\left(F_{7,40}=2.21, p=0.054\right)$. 


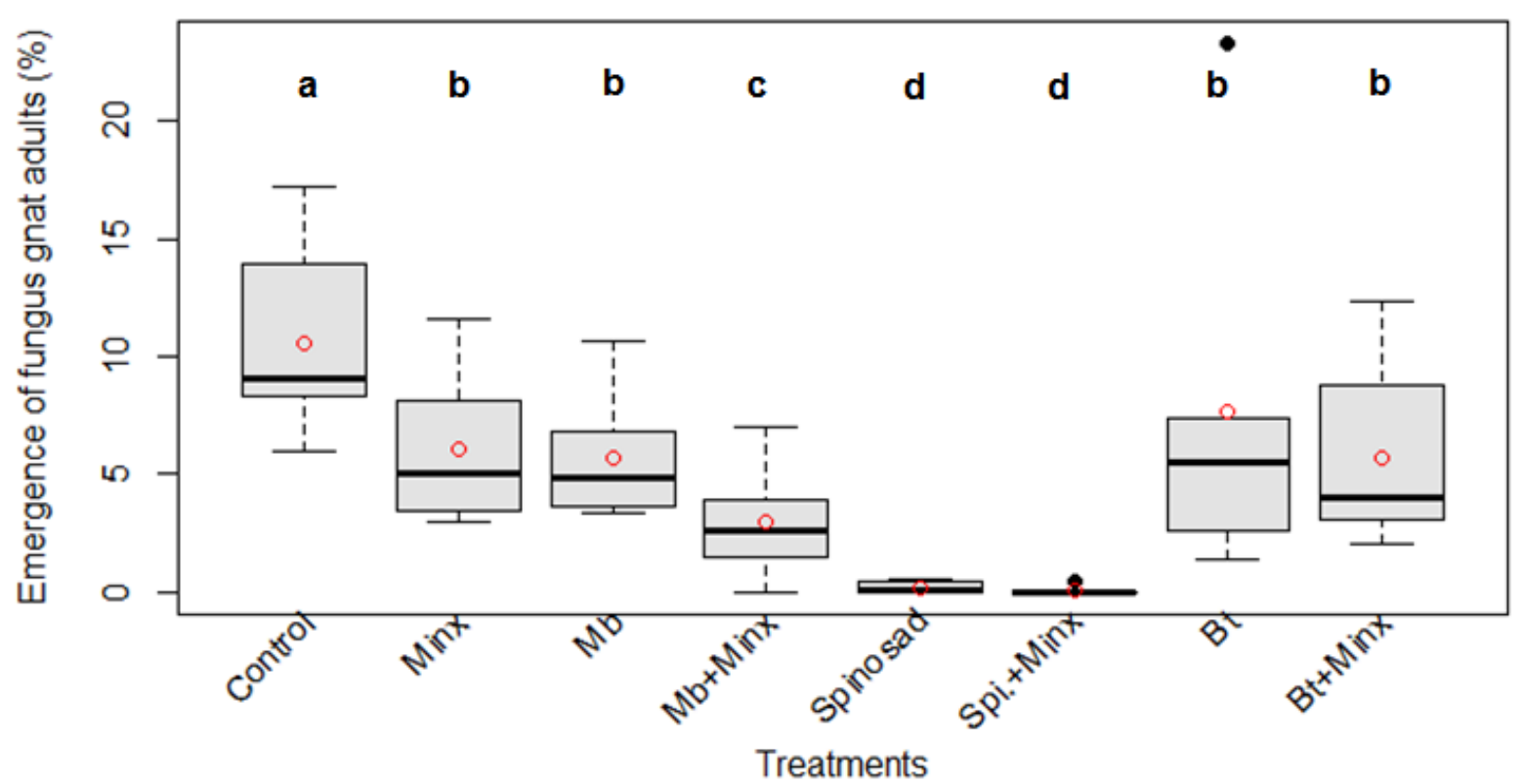

Fig. 4: Emergence of fungus gnat adults (\%) from substrates treated with M. brunneum and two biopesticides (Bt and Spinosad) alone or in combination with the botanical compound. Minx: Botanical capsule containing mint oil; Mb: M. brunneum; Mb + Minx: Combining M. brunneum and botanical capsule; Spi. + Minx: Combining Spinosad and botanical capsule; Bt: Bacillus thuringiensis; Bt + Minx: Combining Bt and botanical capsule; ( $\mathrm{N}=6$ experimental cups / treatment). Different letters indicate significant differences between treatments following Tukey HSD test $\left(F_{7,40}=9.09, p<0.05\right)$.

\section{Experiment 4}

The number of fungus gnat adults hatching from substrates treated with the combination of botanical capsules Minx and M. brunneum $\mathrm{Cb} 15$ was significantly lower than the number of adults hatching from the botanical Minx capsules or the control treatments $\left(F_{24,225}=12.54, p<0.001\right.$; Fig. 5). The adult emergence from substrates treated with the Minx capsules at different rates of mint oil, ranging from $50 \%$ to $68.5 \%$, did not significantly differ between the different rates of the Minx capsules. The Minx capsules with $10 \%$ mint oil at a rate of $150 \mathrm{mg}$ (= Minx $10 \%(150)$ ) and $10 \%$ mint oil at a rate of $50 \mathrm{mg}(=\operatorname{Minx} 10 \%(50))$ attracted more fungus gnat larvae, $65 \%$ and $68.5 \%$ respectively, although not significantly, in comparison to the control treatment $54.5 \%$. The Minx capsules containing $1 \%$ mint oil, specifically at a rate of $50 \mathrm{mg}$ (= Minx $1 \%(50)$ ) were less attractive to fungus gnat larvae (50\%), although not significantly, than the other Minx capsules or 
control treatments. The numbers of emerged adults from the combined applications of $M$. brunneum and Minx capsules were less than the amount of adult hatching from the botanical compound or the control treatments, varying from $15 \%$ (= Mb+Minx $10 \%$ (150)) (mean $\pm \mathrm{SE})$ to $25 \%(=M b+\operatorname{Minx} 10 \%(50))$ (mean $\pm \mathrm{SE})$, with no significant differences between the combined treatments $\left(F_{11,108}=0.32, p=0.98\right)$.

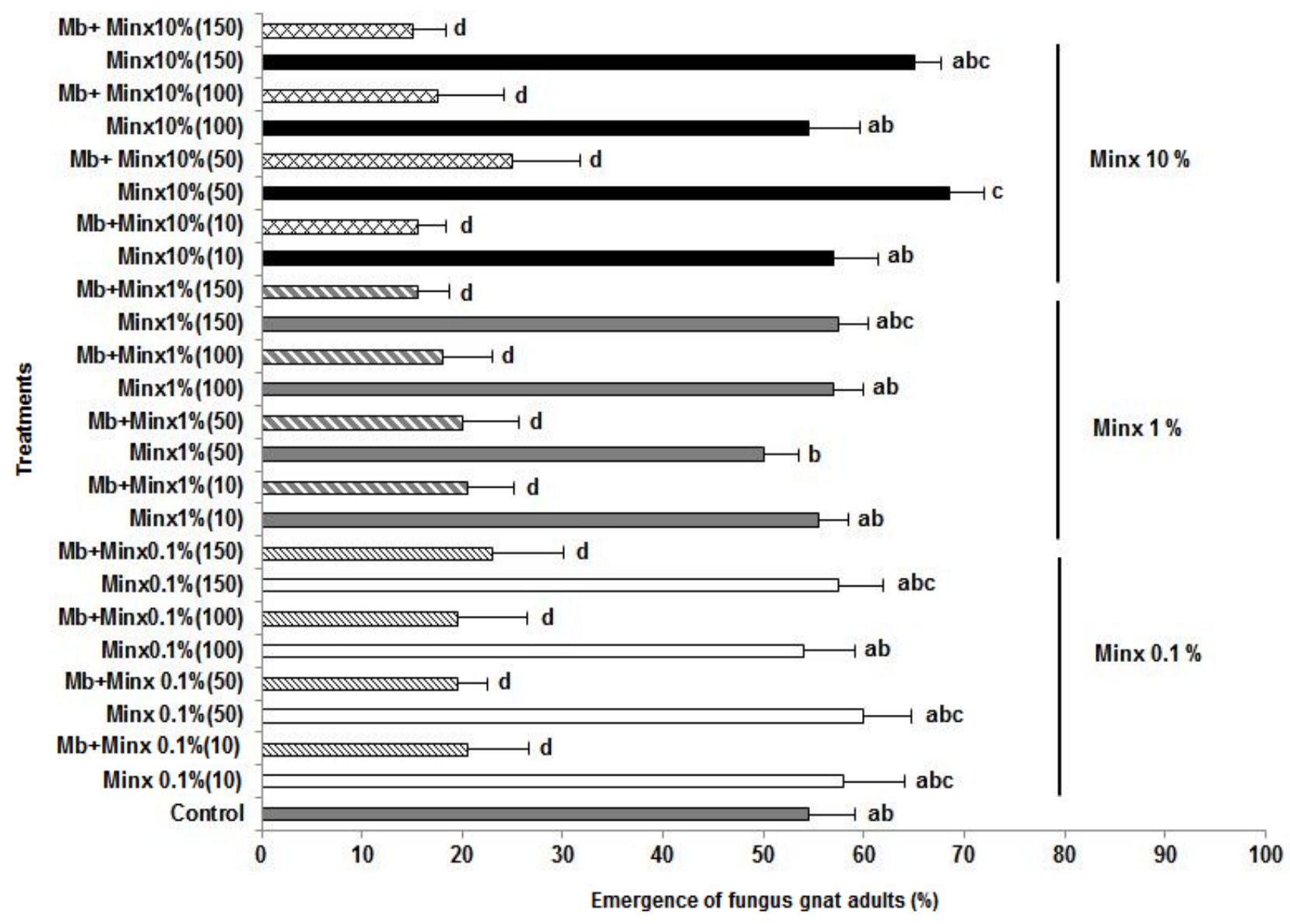

Fig.5: Percentage of fungus gnat adults $( \pm \mathrm{SE}$ ) emerged from larvae exposed to $M$. brunneum alone $(\mathbf{M b})$ or in combination with the botanical capsules $\operatorname{Minx}(\mathbf{M b}+\mathbf{M i n x})$ with three different mint oil concentrations (0.1\%, $1 \%$ and $10 \%)$ at different rates 10, 50, 100 and $150 \mathrm{mg}$. Different letters indicate significant differences as determined by Tukey HSD test $\left(F_{24,225}=12.54, p<0.05\right)$.

\section{Experiment 5}

The application of M. brunneum at a rate of $1 \times 10^{7} \mathrm{spore} / \mathrm{ml}$ and $1 \times 10^{9} \mathrm{spore} / \mathrm{ml}$ individually or in combination with the botanical capsules significantly reduced the emergence of adults compared to the control treatments $\left(F_{14,135}=14.97, p<0.001 ; F i g .6\right)$. The number of adults hatching from the M. brunneum spore suspensions $1 \times 10^{7}$ spore $/ \mathrm{ml}(30.5 \%)$ and $1 \times 10^{9}$ spore $/ \mathrm{ml}(20 \%)$, 
respectively, was significantly lower than the control treatment $(61 \%)\left(F_{2,27}=15.58, p<0.001\right)$. The number of adult emergence from the botanical capsules varied from $53 \%$ for the botanical capsules containing $10 \%$ mint oil to $63.5 \%$ for the capsules with $0.1 \%$ mint oil, with no significant difference between the different rates of the botanical capsules or the control treatments $\left(F_{4,45}=\right.$ $0.68, p=0.61)$. A higher number of adults hatched from larvae exposed to the Minx capsule containing $0.1 \%$ mint oil at a rate of $2 \mathrm{mg}(=\operatorname{Minx} 0.1 \%$ (2)) (63.5\%) as compared to the other botanical compounds or control treatment, indicating a positive interaction with the mint capsules. The data obtained from combining M. brunneum and Minx capsules resulted in fewer number of fungus gnat adults as compared to the single fungal treatment or the control. Conidial suspensions of $M$. brunneum at the rate of $1 \times 10^{9} \mathrm{spore} / \mathrm{ml}$ caused a higher reduction of adult emergence, when applied alone (20\%) or in combination with the botanical compounds, ranging from $15 \%$ to $19 \%$, as compared to the other treatments or the control. The combined application of $M$. brunneum at the rate of $1 \times 10^{9} \mathrm{spore} / \mathrm{ml}$ and Minx capsules indicated an additive effect as shown by the reduced number of adults emerging. However, this effect was not significant in comparison to the single fungal treatment $\left(F_{4,45}=0.28, p=0.88\right)$. In addition, The emergence of adults from larvae exposed to the fungal suspensions of $M$. brunneum at a rate of $1 \times 10^{7} \mathrm{spore} / \mathrm{ml}$ in combination with Minx capsules varied from $16 \%$ (= Mb $10^{7}+\operatorname{Minx} 10 \%$ (2)) to $30.5 \%$ (= Mb $\left.10^{7}+\operatorname{Minx} 0.1 \%(10)\right)$, with no significant differences between these combined treatments and the single fungal treatment $\left(F_{4,45}=2.72, p>0.05\right)$. 


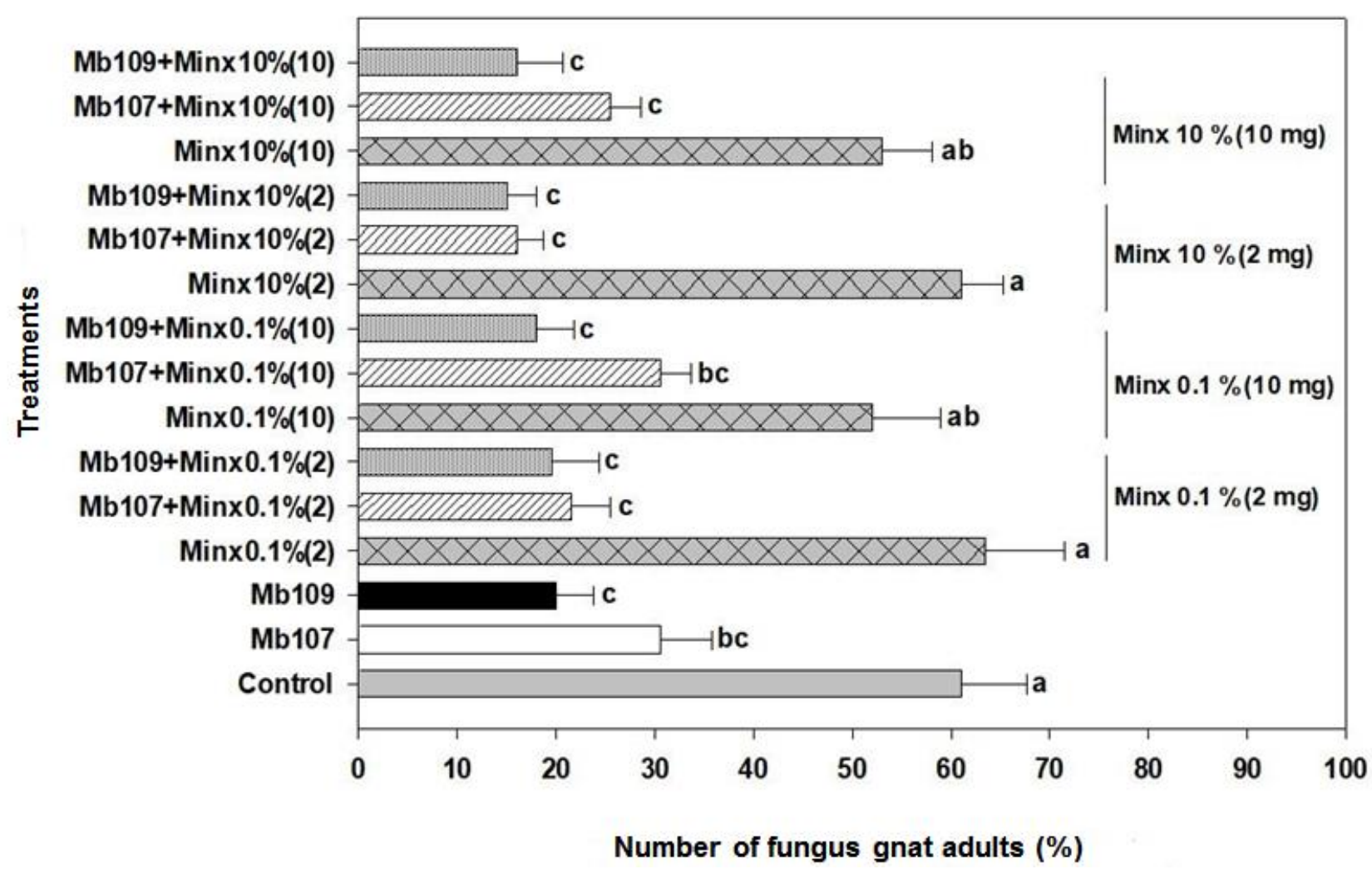

Fig. 6: Percentage of adult emergence $( \pm \mathrm{SE}$ ) from larvae exposed to $M$. brunneum at the rates of $1 \times 10^{7}$ spore $/ \mathrm{ml}\left(\mathbf{M b ~ 1 0 ^ { 7 }}\right)$ and $1 \times 10^{9}$ spore/ml ( $\mathbf{M b ~ 1 0 ^ { 9 }}$ ) individually or in combination with the botanical capsules Minx with two different mint oil concentrations ( $0.1 \%$ and $\mathbf{1 0} \%)$ at a rate of 2 $\mathrm{mg}$ and $10 \mathrm{mg}$. Means followed by a different letter are significantly different (Tukey HSD test $)\left(F_{14,135}=14.97, p<0.05\right)$.

\section{Experiment 6}

The fungal strain $M$. brunneum reduced the number of fungus gnat adults when applied alone or in combination with the Minx capsules as compared to the control $\left(F_{4,45}=2.23, p=0.08\right.$; Fig. 7). A higher percentage of adults hatching from larvae exposed to the Minx capsules (59\%) in comparison to the control treatment $(55.5 \%)$, with no significant difference between the control and Minx treatments $\left(F_{1,18}=0.09, p=0.75\right)$. On the other hand, the substrates treated with $M$. brunneum caused a better effect to control fungus gnats when applied in combination with the botanical compound (40.5\%), although not significantly, compared to M. brunneum alone (52.5 \%). Additionally, the botanical capsules coated with M. brunneum showed an efficient reduction of adult emergence ( $37 \%$ ), although there were no significant differences between this coated Minx 
capsules and the combined application of $M$. brunneum with Minx capsules $\left(F_{1,18}=0.186, p=\right.$ 0.67).

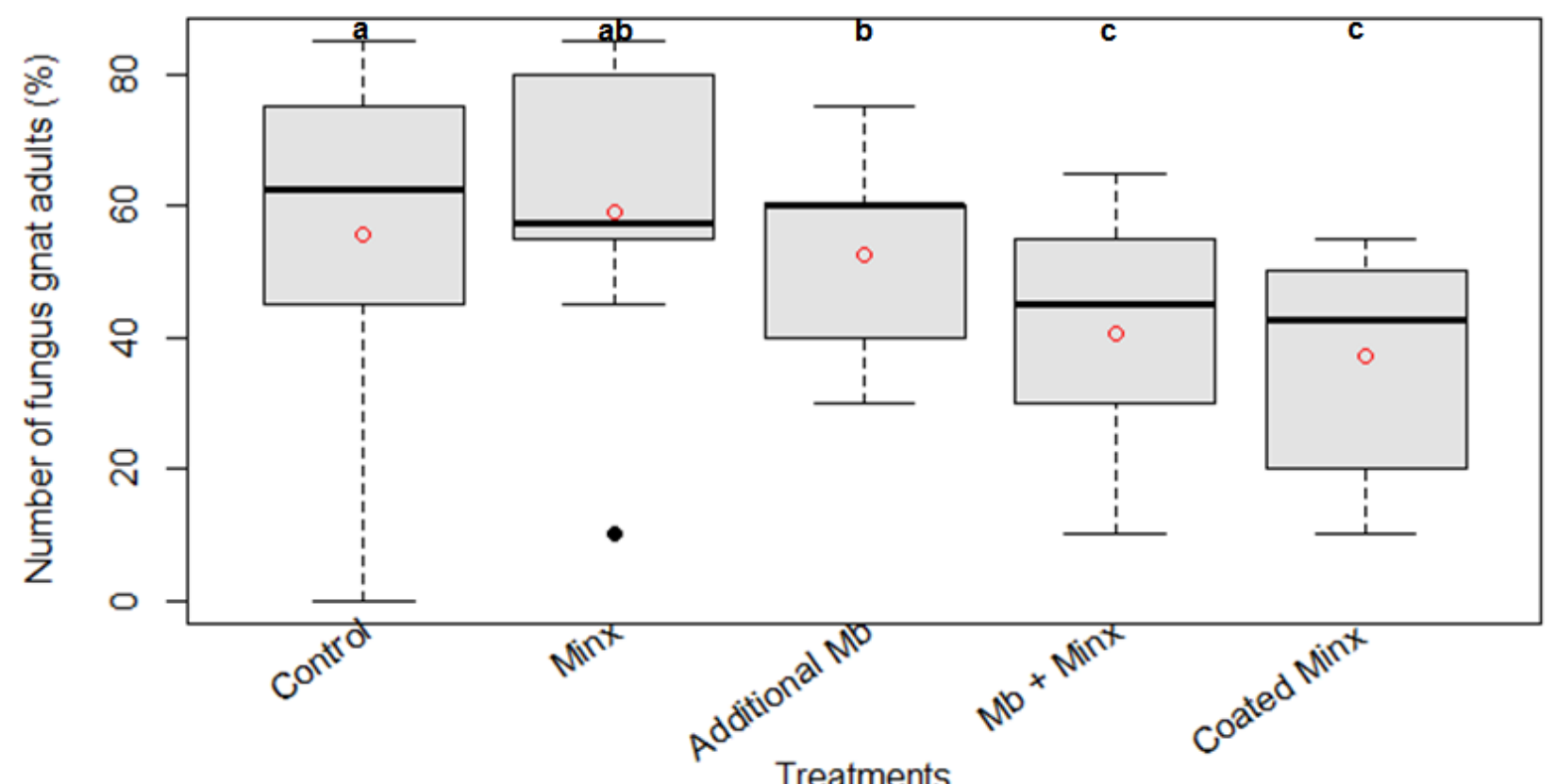

Fig. 7: Emergence of fungus gnat adults (\%) from substrates treated with M. brunneum alone or in combination with the botanical compound Minx. Minx: Botanical compound containing mint oil $0.1 \%$; Coated Minx: the botanical capsule coated with M. brunneum; Additional Mb: the additional fungal suspension of $M$. brunneum after mixing with the Minx capsules; Mb + Minx: Combining M. brunneum and botanical capsule Minx. Different letters indicate significant differences as determined by Tukey HSD test $\left(F_{4,45}=2.23, p<0.05\right)$.

\section{Experiment 7}

In this bioassay, significantly fewer adult numbers emerged from $M$. brunneum and the two biopesticides (Neem and Spinosad) individually or in combination with the botanical capsules as compared to the control treatments $\left(F_{7,72}=16.26, p<0.001\right.$; Fig. 8). Larvae exposed to the fungal and two biopesticides treatments exhibited a high susceptibility to these treatments, resulting in the reduction of adult emergence. The number of emerged adults varied from $3 \%$ for Spinosad treatments to $18.5 \%$ for $M$. brunneum. A higher percentage of adults emerged from substrates treated with the botanical compound Minx (46.5\%) compared to the control treatment (42\%). 
However, there were no significant differences between the botanical capsules and the control in terms of adult emergence $\left(F_{1,18}=0.15, p=0.62\right)$. The combination of the fungal or Spinosad treatments with the botanical capsules resulted in a reduction of adult emergence as shown by the reduced number of adults emerging, although not significantly, in comparison to the single fungal or Spinosad treatments $\left(=M b+\right.$ Minx and $M b: F_{1,18}=0.14, p=0.7 ;$ Spi.+Minx and Spinosad: $\left.F_{1,18}=0.25, p=0.61\right)$. Specifically, Spinosad when applied alone or in combination with the botanical capsule significantly reduced the emergence of adults by $97 \%$ as compared to the other treatments. In addition, the number of fungus gnat adults hatching from substrates treated with Neem or M. brunneum in combination with the botanical capsules was observed $9.5 \%$ and $16 \%$, respectively, with significant differences between these treatments $\left(F_{1,18}=1.94 ; p<0.01\right)$.

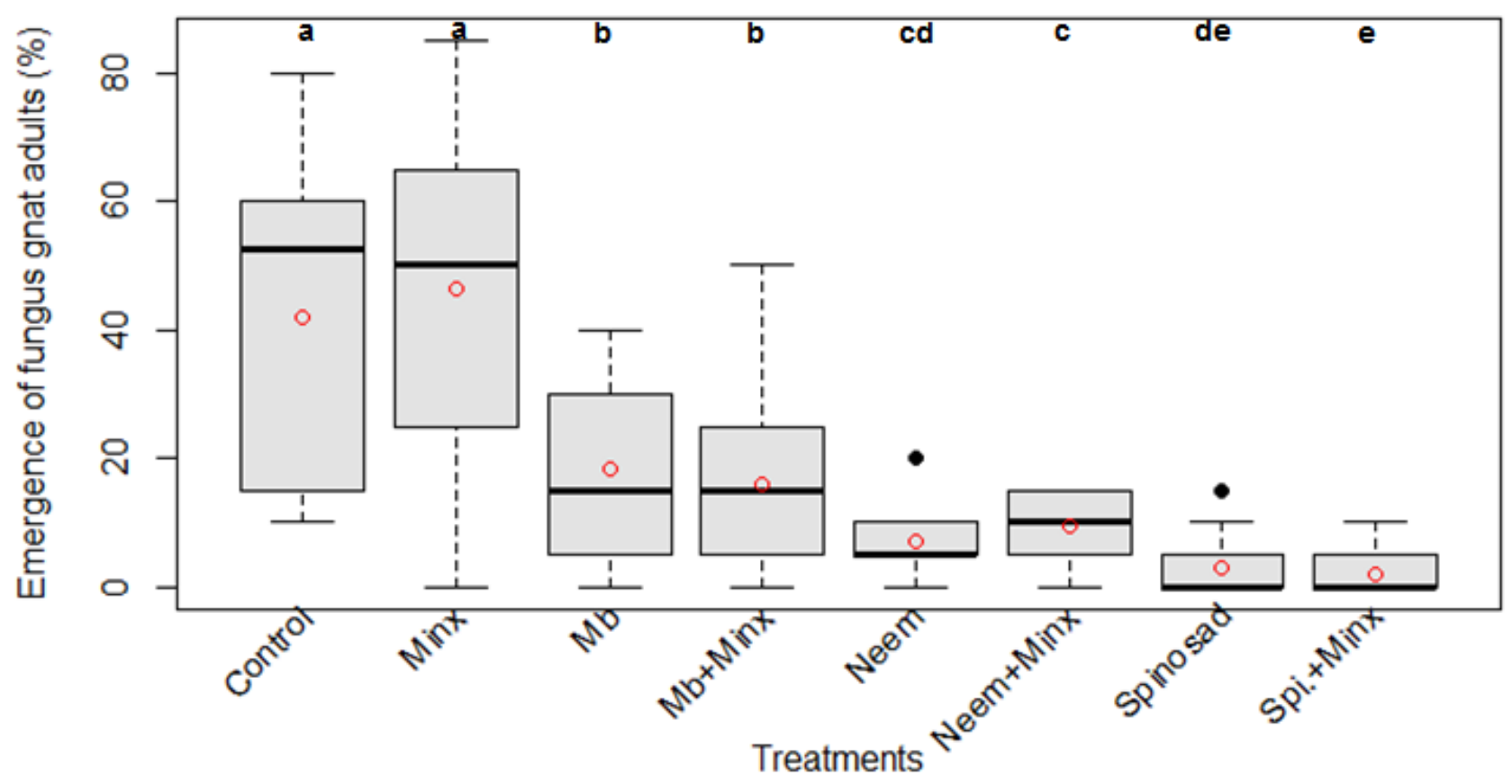

Fig. 8: Emergence of fungus gnat adults (\%) from larvae exposed to the substrates treated with a biopesticide individually or in combination with the botanical capsule Minx. Mb: M. brunneum; Minx: Botanical capsule containing mint oil; Mb + Minx: Combining M. brunneum and a botanical capsule; Neem: Neem Azal ${ }^{\oplus}$; Neem + Minx: Combining Neem Azal ${ }^{\oplus}$ and a botanical capsule; Spi.+Minx: Combining Spinosad and a botanical capsule. Different letters indicate significant differences between treatments following Tukey HSD test $\left(F_{7,72}=16.26, p<0.05\right)$. 


\section{Discussion}

This study builds upon the efficacy of combining a biopesticide and a botanical compound in an "attract and kill" approach targeting fungus gnat larvae. Our study revealed that the fungus gnat larvae were more susceptible to the combined application of fungal or biopesticide treatments and the botanical capsules Minx as compared to single treatments. The combination of Spinosad and the botanical microcapsule Minx resulted in a higher efficacy to control fungus gnat larvae as shown by the reduced emergence of adults $>98 \%$ as compared to the other biopesticide (Neem $A_{z a l^{\oplus}}$ ) and fungal treatments. Furthermore, female fungus gnats preferred the Spinosad-treated cups when applied alone or in combination with the botanical capsules for egg-laying. Interestingly, eggs laid by the females in Spinosad-treated cups could not successfully complete their life cycle to adults, resulting in an efficient reduction of adult emergence. Therefore, the combination of Spinosad and the botanical capsule Minx might be used as a "trap crop strategy".

\section{Egg-laying preference of fungus gnats}

Fungus gnat females preferred to lay more eggs, although not significantly, in Spinosad-treated cups individually or in combination with the botanical capsules as compared to the fungal treatments or the control. This finding might be supported by Perez et al., (2007) who demonstrated that Spinosad has a distinctive aroma derived from Actinomycetes which was attractive to the females of Aedes aegypti (Diptera: Culicidae). Additionally, the increased egglaying by fungus gnat females was probably a result of Spinosad poisoning. Given these results, Toews \& Subramanyam (2003) demonstrated the enhanced egg-laying behavior by Rhyzopertha dominica in Spinosad treatments. Spinosad acts on the nicotinic acetylcholine and gammaaminobutyric acid receptor sites of nerve cells, causing the poisoning symptoms in insects including leg extensions, wing beating, air swallowing, protruding of the ovipositor and expelling of the eggs (Salgado, 1998). Similarly, Scott (1998) reported the protrusion of the ovipositor of houseflies when exposed to Spinosad. This is the first study that examined the egg-laying behavior of fungus gnat females when exposed to Spinosad, and we found that the eggs laid by females in Spinosad-treated cups could not successfully finalize their life cycle to adults, resulting in an efficient reduction of adult emergence. Specifically, the combination of Spinosad and the botanical capsules was highly attractive for oviposition of fungus gnats; thus, this combination might be used as a "trap crop strategy" in greenhouse management. Fungus gnats are kept away from the main crops in the greenhouse and lured to trap pots containing Spinosad and the botanical 
capsules. However, further investigations are required to implement this strategy under greenhouse conditions.

Additionally, fungus gnat females showed a strong ovipositional preference on the substrate treated with botanical capsules vs. control substrate. This attractive behavior of fungus gnats to oviposit may be due to the volatiles emitted from the botanical capsule added to the growing medium. In this regard, Meers \& Cloyd (2005) reported that the egg-laying behavior of fungus gnat females rely on the volatiles emitted from the growing medium. The components of the growing medium might be regarded as a key factor that determines where fungus gnats lay eggs (Evans et al., 1998). In addition, volatiles generated as a result of microbial activity might be implicated as oviposition stimulants for fungus gnat adults (Olson et al., 2002). For instance, Frouz \& Naváková (2001) reported the egg-laying preference of the fungus gnat species $L$. ingenua when exposed to fungal strains of Trichoderma spp. However, the ovipositional preference of fungus gnat species to various fungi is highly variable, even among species of the same genus (Frouz \& Naváková, 2001). In addition, Braun et al. (2012) found a strong ovipositional preference of fungus gnat females to an entomopathogenic fungi (EPF) B. bassiana. However, the oviposition site selection by sciarid fungus gnat was reported to be a complex process, a number of different biological, physical and chemical factors involved (Braun et al., 2012). In our study, fungus gnat females were attracted to oviposit in the substrates treated with M. brunneum alone or in combination with the botanical capsules. This behavior may be due to the volatiles generated from this microbial agent alone or in combined with the Minx capsules, which have been shown an attractive stimulant for fungus gnat adults. However, the eggs laid by the females on M. brunneum alone or in combination with the botanical capsules could not fully develop to adults, resulting in a reduced number of adults emerging.

Influence of botanical compounds at different rates on larval fungus gnat B. impatiens

The performance of the botanical capsules varied according to the different concentrations of mint oil embedded in capsules, with no significant differences between the botanical capsules. The botanical Minx capsules containing mint oil $(0.1 \%)$ have been found to be highly attractive for fungus gnat larvae of $B$. impatiens, which exhibited a positive effect on the development of the fungus gnat larvae. The botanical capsules Minx with $10 \%$ mint oil also indicated an attractant effect for fungus gnat larvae as observed with Minx capsules containing $0.1 \%$ mint oil. Therefore, the botanical capsules with $0.1 \%$ mint oil was chosen as an optimal rate for the attractant of 
fungus gnat larvae in our trials. According to Brattsten (1983), the most insects, when exposed the botanical compounds, might exhibit neurotoxic symptoms such as agitation, hyperactivity and even paralysis.

Additionally, other components applied in the structure of the botanical capsules such as sodium alginate used for encapsulation might have caused a positive cue on fungus gnat larvae. In this regard, Ruiz-Vega et al. (2018) demonstrated a good biocompatibility of sodium alginate with biological products, which resulted in a positive effect on the survivorship and infectivity of the biological product. Additionally, Heuskin et al. (2012) demonstrated that semiochemical alginate beads can be considered as an efficient slow-release formulation in a biological control approach and could be useful to attract insects (e.g. aphid parasitoids).

\section{Efficacy of biopesticides on the control of fungus gnat larvae}

M. brunneum and biological insecticides tested caused a high reduction in the emergence of fungus gnat adults. In this study, fungus gnat larvae have been found to be susceptible to infection with $M$. brunneum, which resulted in a suppression of adult emergence as compared to the control treatments. This reduction of hatching adults from $M$. brunneum treatment could be due to a number of factors, including secretion of toxic secondary metabolites (Vey et al., 2001), negative effect of Metarhizium spp. via muscle paralysis and death (Samuels et al., 1998), transferring larvae from stock-rearing medium, or intrinsic unidentified biological factors (Olson et al., 2002), respectively. In a similar work, Tavoosi Ajvad et al. (2019) indicated a > $50 \%$ larval mortality of the fungus gnat L. auripila after exposure to $M$. anisopliae.

The two biopesticides (Neem Azal ${ }^{\circledR}$ and Spinosad) tested were able to significantly reduce the numbers of fungus gnat adults. This result is agreement with the report of Ludwig \& Oetting (2001), which found that larvae of the fungus gnat B. coprophila were susceptible to infection by azadirachtin, resulting in a reduction of fungus gnat emergence. Similarly, Cloyd (2008) reported the susceptibility of larvae of the fungus gnat Bradysia spp. when exposed to the neem products. Azadirachtin is available commercially and recommended for the control of the fungus gnats such as B. impatiens by producers (Boeke et. al., 2004, Jänsch et al., 2018). It has been reported that azadirachtin is physiologically able to inhibit the synthesis and release of ecdysteroids, resulting in incomplete moulting of immature insects (Mordue \& Nisbet, 2000). In addition, azadirachtin might weaken the defense systems of larvae and act as a growth regulator (Su \& Mulla, 1998). The other biological insecticide Spinosad tested in this study was more toxic to fungus gnat larvae than 
Neem Azal, as shown by the reduced number of emerged adults. The reduction of adults hatching from Spinosad in the present study could be due to the mechanism of action of Spinosad, which affects the nicotinic acetylcholine receptors in the insect nervous system, resulting in disruption of normal nerve function and death (Orr et al., 2009). Cloyd (2009) reported that Spinoad perform rapidly and is able to kill thrips, one of common pests on the ornamental crops in greenhouses, within some days after either contact or ingestion. In similar work, Perez et al. (2007) reported an efficient reduction (94-100\%) of survival larvae of $A$. aegypti (L.) (Diptera: Culicidae) when exposed to a Spinosad treatment. The effect of Spinosad on the adult emergence of fungus gnat $B$. impatiens has not been previously considered. Hence, the efficacy of this bioinsecticide needs further investigations to develop a more complement management program for fungus gnat control.

Efficacy of combined application of a biopesticide and a botanical compound on fungus gnat larvae The application of the fungal strain M. brunneum or Spinosad in combination with the botanical capsules Minx has provided an additive effect as shown by the reduced number of adult emergence as compared to the single treatments. Spinosad in combination with the Minx capsules caused a slightly higher reduction of adult emergence by $98 \%$, although not significantly, as compared to Spinosad alone. This reduction might be due to the hyperactivity and the emigration of B. impatiens larvae towards the botanical capsules (Minx) (= an attractive agent) through the substrate; thus increasing the probability of larvae to be infected by a fungal spore or Spinosad suspensions applied in the substrate via contact or ingestion application. Hence, this combined formulation might lead to a better efficacy to control fungus gnats in comparison to a single treatment. Moreover, the reduction of adult emergence could be influenced by a number of factors such as changes of the larval diet, intrinsic unidentified biological factors as demonstrated previously (Olson et al., 2002, Main et al., 2014), and larval cannibalism in specific conditions (Wilkinson \& Daugherty, 1970a).

The combination of biological agents to achieve a better effect might result in an efficient approach for insect pest management in agricultural systems. In this regard, Tavoosi Ajvad et al. (2019) tested the simultaneous application of M. anisopliae and a predatory mite, G. aculeifer in mushroom cultures for controlling the fungus gnat species L. auripila. In another study, the combination of an EPN ( $H$. indica) and the predatory mite (S. scimitus) exhibited a high mortality (73\%) in B. impatiens larvae (Acharya et al., 2019). The co-application of an EPN (Steinernema 
feltiae) with insecticide (imidacloprid) could increase the control efficacy against $B$. odoriphaga in chive fields (Yan et al., 2019). Additionally, the combination of the EPN S. feltiae and thiamethoxam has been shown to be highly effective (94.96 \%) for controlling $B$. odoriphaga larvae in integrated pest management (Wu et al., 2017). In our study, biopesticides (M. brunneum and Spinosad), specifically Spinosad in combination with the botanical capsules Minx (as an attractive agent) have been found to be highly effective (>90\%) in reducing the adult emergence of $B$. impatiens. These results pointed to an "attract and kill" effect, which might efficiently reduce fungus gnat populations. This study provides the basis for further investigations, which should be conducted in more realistic conditions. Further investigations are needed to evaluate the effect of these combined formulations under more realistic conditions.

\section{Conclusion}

This is the first report that examined the efficacy of the biopesticides ( $M$. brunneum or Spinosad) in combination with a botanical compound to control fungus gnat. This study revealed the potential of these combined formulations as an "attract and kill" strategy to control fungus gnats. The combined application of biopesticides, specifically Spinosad and a botanical compound Minx resulted in an efficient reduction of adult emergence. Moreover, we found a strong egg-laying behavior of female fungus gnats when exposed to Spinosad-treated cups, but these eggs laid by females could not fully develop to adults. This result pointed to a new approach, named here as a "trap crops strategy" to reduce the fungus gnat population. Further studies are needed to evaluate the effectiveness of these combined formulations to suppress fungus gnats under greenhouse conditions.

\section{Acknowledgements}

We would like to thank Bianca Tappe and Marie Nörthemann for their technical assistance. We would like to thank Jörg Rademacher (Katz Biotech AG, Baruth, Germany) for providing the botanical capsules.

\section{References}

Acharya, R., Hwang, H. S., Shim, J. K., Yu, Y. S. \& Lee, K. Y. (2019) Control efficacy of fungus gnat, Bradysia impatiens, enhanced by a combination of entomopathogenic nematodes and predatory mites. Biological Control, 138. 
Baker, D. K., Rice, S. J., Leemon, D. M. \& James, P. J. (2018) Horizontal transmission of Metarhizium anisopliae (Hypocreales: Clavicipitacea) and the effects of infection on oviposition rate in laboratory populations of Musca domestica (Diptera: Muscidae). Pest Management Science, 74, 987-991.

Bethke, J. A. \& Dreistadt, S. H. (2013) Pest notes: Fungus gnats. University of California Statewide Integrated Pest Management Program, Davis, CA.

Boeke, S. J., Boersma, M. G., Alink, G. M., Van Loon, J. J., Van Huis, A., Dicke, M. \& Rietjens, I. M. (2004) Safety evaluation of neem (Azadirachta indica) derived pesticides. Journal of Ethnopharmacology, 94, 25-41.

Brattsten, L. B. (1983) Cytochrome P-450 involvement in the interactions between plant terpenes and insect herbivores. In: Hedin, P. A. (eds.), Plant Resistance to Insects. ACS Symposium Series Vol. 208, American ChemicalSociety, Washington, D. C. 173-195.

Braun, S. E., Sanderson, J. P., Daughtrey, M. L. \& Wraight, S. P. (2012) Attraction and oviposition responses of the fungus gnat Bradysia impatiens to microbes and microbe-inoculated seedlings in laboratory bioassays. Entomologia Experimentalis et Applicata, 145, 89-101.

Braun, S. E., Sanderson, J. P. \& Wraight, S. P. (2012) Larval Bradysia impatiens (Diptera: Sciaridae) potential for vectoring Pythium root rot pathogens. Phytopathology, 102, 283-289.

Butt, T. M., Jackson, C. \& Magan, N. (2001) Fungi as biocontrol agents: progress, problems and potential. CABI Publishing, Wallingford, UK, 390p.

Cabrera, A. R., Cloyd, R. A. \& Zaborski, E. R. (2003) Effect of monitoring technique in determining the presence of fungus gnat, Bradysia spp. (Diptera: Sciaridae), larvae in growing medium. Journal of Agricultural and Urban Entomology, 20, 41-47.

Carney, V. A., Diamond, J. C., Muphy, G. D. \& Marshall, D. (2002). The potential of Atheta coriaria Kraatz (Colepotera: staphylinidae) as a biological control agent for use in greenhouse crops. IOBC/WPRS Bulletin, 25, 37-40.

Cleveland, C. B., Mayes, M. A. \& Cryer, S. A. (2001) An ecological risk assessment for Spinosad use on cotton. Pest Management Science, 58, 70-84.

Cloyd, R. A. (2000) Fungus gnat and shore fly management strategies: panel discussion. In: Proceedings for the $16^{\text {th }}$ conference on insect and disease management on ornamentals. San Jose, CA. Society of American Florists, Alexandria, VA. 
Cloyd, R. A. \& Zaborski, E. R. (2004) Fungus gnats, Bradysia spp. (Diptera: Sciaridae), and other arthropods in commercial bagged soilless growing media and rooted plant plugs. Journal of Economic Entomology, 97, 503-510.

Cloyd, R. A. \& Dickinson, A. (2006) Effect of Bacillus thuringiensis subsp. israelensis and neonicotinoid insecticides on the fungus gnat Bradysia sp. nr. Coprophila (Lintner) (Diptera: Sciaridae). Pest Management Science, 62, 171-177.

Cloyd, R. A. (2008) Management of fungus gnats (Bradysia Spp.) in greenhouses and nurseries. Floriculture and Ornamental Biotechnology, 2, 84-89.

Cloyd, R. A. (2009) Western flower thrips (Frankliniella occidentalis) management on ornamental crops grown in greenhouses: Have we reached an impasse. Pest Technology, 3, 1-9.

Copping, L. G. \& Menn, J. J. (2000) Biopesticides: a review of their action, applications and efficacy. Pest Management Science, 56, 651-676.

Dow AgroSciences. (2001) Spinosad technical bulletin. Dow AgroSciences LLC, Indianapolis, IN. http://www.parapro.com/documents/Spinosad_technical_bulletin.pdf.

Dreistadt, S. H. (2001) Fungus gnats, shore flies, moth flies, and march flies. In: Pest Notes. Publication 7448. University of California, Davis, CA.

El-Hamalawi, Z. A. (2008) Acquisition, retention and dispersal of soilborne plant pathogenic fungi by fungus gnats and moth flies. Annals of Applied Biology, 153, 195-203.

El-Sayed, A. M., Suckling, D. M., Byers, J. A., Jang, E. B. \& Wearing, C. H. (2009) Potential of 'lure and kill' in long-term pest management and eradication of invasive species. Journal of Economic Entomology, 102, 815-835.

Erler, F., Polat, E., Demir, H., Cetin, H. \& Erdemir, T. (2009) Evaluation of microbial products for the control of the mushroom phorid fly, Megaselia Halterata (Wood). Journal of Entomological Science, 44, 89-97.

Evans, M. R., Smith, J. N. \& Cloyd, R. A. (1998) Fungus gnat population development in coconut coir and Sphagnum peat-based substrate. HortTechnology, 8, 406-409.

Frouz, J. \& Naváková, A. (2001) A new method for rearing the sciarid fly, Lycoriella ingenua (Diptera: Sciaridae), in the laboratory: possible implications for the study of fly-fungal interactions. Pedobiologia, 45, 329-340.

Gillespie, D. R. \& Menzies, J. G. (1993) Fungus gnats vector Fusarium oxysporum f. Sp. Radicislycopersici. Annals of Applied Biology, 123, 539-44. 
Golo, P. S., Gardner, D. R., Grilley, M. M., Takemoto, J. Y., Krasnoff, S. B., Pires, M. S., Fernandes, E. K. K., Bittencourt, V. R. E. P. \& Roberts, D. W. (2014) Production of destruxins from Metarhizium spp. fungi in artificial medium and in endophytically colonized cowpea plants. PLoS One, 9, e104946.

Hamlen, R. A. \& Mead, F. W. (1979) Fungus gnat (Diptera: Sciaridae) larval control in greenhouse plant production. Journal of Economic Entomology, 72, 269-271.

Harris, M. A., Oetting, R. D. \& Gardner, W. A. (1995) Use of entomopathogenic nematodes and a new monitoring technique for control of fungus gnats, Bradysia Coprophila (Diptera: Sciaridae), in floriculture. Biological Control, 5, 412-418.

Heuskin, S., Lorge, S., Godin, B., Leroy, P., Frère, I., Verheggen, F. J., Haubruge, E., Wathelet, J. P., Mestdagh, M., Hance, T. \& Lognay, G. (2012) Optimisation of a semiochemical slow-release alginate formulation attractive towards Aphidius ervi Haliday parasitoids. Pest management science, $68,127-136$.

Jackson, M. A., Dunlap, C. A. \& Jaronski, S. T. (2010) Ecological considerations in producing and formulating fungal entomopathogens for use in insect biocontrol. BioControl, 55, 129-145.

Jänsch, S., Bauer, J., Leube, D., Otto, M., Römbke, J., Teichmann, H. \& Waszak, K. (2018) A new ecotoxicological test method for genetically modified plants and other stressors in soil with the black fungus gnat Bradysia impatiens (Diptera): current status of test development and dietary effects of azadirachtin on larval development and emerge. Environmental Sciences Europe, 30, 1-12.

Jagdale, G. B., Casey, M. L., Grewal, P. S. \& Lindquist, R. K. (2004) Application rate and timing, potting medium, and host plant effects on the efficacy of Steinernema feltiae against the fungus gnat, Bradysia coprophila, in floriculture. Biological Control, 29, 296-305.

James, R. L., Dumroese, R. K. \& Wenny, D. L. (1995) Botrytis cinerea carried by adult fungus gnats (Diptera: Sciaridae) in container nurseries. Tree Planters Notes, 46, 48-53.

Jelinek, S. \& Azzopardi, S. (2010) Fungus gnat management in greenhouse crops. Primefact 1006. New South Wales Department of Industry and Investment.

Jiang, Y. \& Mulla, M. S. (2006) Susceptibility of the adult eye gnat Liohippelates collusor (Diptera: Chloropidae) to neonicotinoids and Spinosad insecticides. Journal of Vector Ecology, 31, 6570. 
Kennedy, M. K. (1974) Survival and development of Bradysia impatiens (Diptera: Sciaridae) on fungal and non-fungal food sources. Annals of the Entomological Society of America, 67, 745749.

Kirst, H. A., Michel, K. H., Mynderse, J. S., Chao, E. H., Yao, R. C., Nakatsukasa, W. M., Boeck, L. D., Occlowitz, J., Paschel, J. W., Deeter, J. B. \& Thompson, G. D. (1992) Discovery, isolation and structure elucidation of a family of structurally unique fermentation-derived tetracyclic macrolides. In: Baker, D. R., Fenyes, J. G. \& Steffens, J. J. (eds.), Synthesis and chemistry of agrochemicals III. American Chemical Society, Washington, D. C. 214-225.

Koller, M. (2011) Comparison of steinernema feltiae, bacillus thuringiensis israelenis and azadirachtin to control sciarids in organic potted herbs. Acta horticulturae, 915, 179-184.

Lacey, L. A., Frutos, R., Kaya, H. K. \& Vail, P. (2001) Insect pathogens as biological control agents: do they have a future? Biological Control, 21, 230-248.

Leath, K. T. \& Newton, R. C. (1969) Interaction of a fungus gnat, Bradysia sp. (Sciaridae) with Fusarium spp. on alfalfa and red clover. Phytopathology, 59, 257-258.

Ludwig, S. W. \& Oetting, R. D. (2001) Evaluation of medium treatments for management of Frankliniella occidentalis (Thripidae: Thysanoptera) and Bradysia coprophila (Diptera: Sciaridae). Pest Management Science, 57, 1114-1118.

Main, M., McCaffrey, J. P. \& Morra, M. J. (2014) Insecticidal activity of Brassica juncea seed meal to the fungus gnat Bradysia impatiens Johannsen (Diptera:Sciaridae). Journal of Applied Entomology, 138, 701-707.

Meers, T. L. \& Cloyd, R. A. (2005) Egg-laying preference of female fungus gnat Bradysia sp. nr. coprophila (Diptera: Sciaridae) on three different soilless substrates. Journal of Economic Entomology, 98, 1937-1942.

Mertz, F. P. \& Yao, R. C. (1990) Saccharopolyspora spinosa sp. nov. isolated from soil collected in a sugar rum still. International Journal of Systematic Bacteriology, 40, 34-39.

Mordue, A. J. \& Nisbet, A. J. (2000) Azadirachtin from the neem tree Azadirachta indica: its action against insects. Anais da Sociedade Entomológica do Brasil, 29, 615-632.

Olson, D. L., Oetting, R. D. \& Van lersel, M. W. (2002) Effect of soilless potting media and water management on development of fungus gnats (Diptera: Sciaridae) and plant growth. HortScience, 37, 919-923. 
Orr, N., Shaffner, A. J., Richey, K. \& Crouse, G. D. (2009) Novel mode of action of Spinosad: receptor binding studies demonstrating lack of interaction with known insecticide target sites. Pesticide Biochemistry and Physiology, 95, 1-5.

Osborne, L. S., Boucias, D. G. \& Lindquist, R. K. (1985) Activity of Bacillus thuringiensis var. israelensis on Bradysia coprophila (Diptera: Sciaridae). Journal of Economic Entomology, 78, 922-925.

Paula, A. R., Silva, L. E. I., Ribeiro, A., Butt, T. M., Silva, C. P. \& Samuels, R. I. (2018) Improving the delivery and efficiency of fungus-impregnated cloths for control of adult Aedes aegypti using a synthetic attractive lure. Parasites \& Vectors, 11, 285-294.

Perez, C. M., Marina, C. F., Bond, J. G., Rojas, J. C., Valle, J. \& Williams, T. (2007) Spinosad, a naturally derived insecticide, for control of Aedes aegypti (Diptera: Culicidae): efficacy, persistence, and elicited oviposition response. Journal of Medical Entomology, 44, 631-638.

Popko, D. A., Henke, J. A., Mullens, B. A. \& Walton, W. E. (2018) Evaluation of two entomopathogenic fungi for control of Culex quinquefasciatus (Diptera: Culicidae) in underground storm drains in the Coachella Valley, California, United States. Journal of Medical Entomology, 55, 654-665.

Poprawski, T., Robert, P. H. \& Maniania, N. (1994) Contact toxicity of the mycotoxin destruxin E to Empoasca vitis (Göthe)(Hom., Cicadellidae). Journal of Applied Entomology, 117, 135-143.

Ruiz-Vega, J., Cortés-Martínez, C. I. \& García-Gutiérrez, C. (2018) Survival and infectivity of entomopathogenic nematodes formulated in sodium alginate beads. Journal of Nematology, $50,273-280$.

Salgado, V. L. (1998) Studies on the mode of action of Spinosad: insect symptoms and physiological correlates. Pesticide Biochemistry and Physiology, 60, 91-102.

Samuels, R. I., Reynolds, S. E. \& Charnley, A. K. (1998) Calcium channel activation of insect muscle by destruxins, insecticidal compounds produced by entomopathogenic fungus Metarhizium anisopiliae. Comparative Biochemistry and Physiology - Part C: Toxicology and Pharmacology, $90,403-412$.

Scott, J. G. (1998) Toxicity of Spinosad to susceptible and resistant strains of house flies, Musca domestica. Pesticide Science, 54, 131-133.

Stanton, G. \& Sanders, J. (1998) Ball identification guide to greenhouse pests and beneficials. Batavia, IL, USA: Ball Publishing (Grower Talks Bookshelf). 
Su, T. \& Mulla, M. S. (1998) Ovicidal activity of neem products (azadirachtin) against Culex tarsalis and Culex quinquefasciatus (Diptera: Culicidae). Journal of the American Mosquito Control Association, 14, 204-209.

Tavoosi Ajvad, F., Madadi, H., Michaud, J. P., Zafari, D. \& Khanjani, M. (2019) Combined applications of an entomopathogenic fungus and a predatory Mite to control fungus gnats (Diptera: Sciaridae) in mushroom production. Biological Control, 141.

Thompson, G. D., Michael, H. H., Yao, R. C., Mynderse, J. S., Mosburg, C. T., Wordsen, T. V., Chio, E. H., Sparks, T. C. \& Hutchins, S. H. (1997) The discovery of Saccharopolyspora spinosa and a new class of insect control products. Down to Earth, 52, 1-5.

Toews, M. D. \& Subramanyam, B. (2003) Contribution of contact toxicity and wheat condition to mortality of stored-product insects exposed to Spinosad. Pest Management Science, 59, 538544.

Vega, F. E., Posada, F., Aime, M. C., Pava-Ripoll, M., Infante, F. \& Rehner, S. A. (2008) Entomopathogenic fungal endophytes. Biological Control, 46, 72-82.

Vey, A., Hoagland, R. \& Butt, T. M. (2001) Toxic metabolites of fungal biocontrol agents. In: Butt, T. M., Jackson, C. W. \& Magan, N. (eds.) Fungi as biocontrol agents progress, problems and potential. CABI Publishing, Wallingford, UK, 311-346.

Vincent, C., Goettel, M. S. \& Lazarovits, G. (2007) Biological Control: a Global Perspective. CAB International/AAFC, Wallingford, United Kingdom.

Wilkinson, J. D. \& Daugherty, D. M. (1970a) The biology and immature stages of Bradysia impatiens (Diptera: Sciaridae). Annals of the Entomological Society of America, 63, 656-660.

Woltz, J. M., Donahue, K. M. Bruck, D. J. \& Lee, J. C. (2015) Efficacy of commercially available predators, nematodes and fungal entomopathogens for augmentative control of Drosophila suzukii. Journal of Applied Entomology, 139, 759-770.

Wright, E. M., \& Chambers, R. J. (1994) The biology of the predatory mite Hypoaspis miles (Acari: Laelapidae), a potential biological control agent of Bradysia paupera (Diptera: Sciaridae). Entomophaga, 39, 225-235.

Wu, H., Gong, Q., Fan, K., Sun, R., Xu, Y. \& Zhang, K. (2017) Synergistic effect of entomopathogenic nematodes and thiamethoxam in controlling Bradysia odoriphaga Yang and Zhang (Diptera: Sciaridae). Biological Control, 111, 53-60. 
Yan, X., Zhao, G. Y. \& Han, R. C. (2019). Integrated management of chive gnats (Bradysia odoriphaga Yang \& Zhang) in chives using entomopathogenic nematodes and low-toxicity insecticides. Insects, 10, 161.

Yee, W. L. (2018) Spinosad versus spinetoram effects on kill and oviposition of Rhagoletis indifferens (Diptera: Tephritidae) at differing fly ages and temperatures. Journal of Insect Science, 18. 
Chapter 4

Combined application of a biopesticide and a botanical compound to control fungus gnats Bradysia impatiens on different host plants

Mahsa Dehghani*, Jörg Rademacher and Stefan Vidal

* Corresponding author 


\title{
Combined application of a biopesticide and a botanical compound to control
}

\section{fungus gnats Bradysia impatiens on different host plants}

M. Dehghani ${ }^{1 *}$, J. Rademacher ${ }^{2}$ and S. Vidal ${ }^{1}$

${ }^{1}$ Georg-August-Universität Göttingen, Department of Crop Sciences, Agricultural Entomology, Grisebachstrasse 6, 37077 Göttingen, Germany

${ }^{2}$ Katz Biotech AG, Baruth, Germany

*Corresponding author: E-mail address: mdehgha@gwdg.de

\begin{abstract}
Fungus gnats, Bradysia impatiens (Diptera: Sciaridae) are one of the most common pests of greenhouses and nurseries. We assessed the effectiveness of two biopesticides (Metarhizium brunneum isolate $\mathrm{Cb} 15$ and Spinosad) individually or in combination with a botanical compound against fungus gnats $B$. impatiens using three different host plant species (basil, parsley and poinsettia), under greenhouse conditions. Eighty fungus gnat adults were released into each experimental chamber with potted plants and the soils drenched with the biopesticides individually or in combination with botanical capsules. $48 \mathrm{~h}$ after exposure, the number of eggs laid by the females were recorded. Using basil plants, fungus gnat females preferred to lay more eggs, although not significantly, in these soils treated with the botanical capsules $(65.28 \pm 12.79)$ or the control treatment $(60.25 \pm 16.61)$ compared to the other treatments. In addition, females exhibited a strong ovipositional preference for parsley and poinsettia plant soils treated with Spinosad individually or in combination with botanical capsules compared to the other treatments. In subsequent bioassay, the combination of these biopesticides with the botanical compound showed that in the host plants species tested so far, Spinosad treatments individually or in combination with the botanical capsules significantly reduced the number of emerged fungus gnat adults as compared to the fungal or the control treatments. Specifically, in poinsettia plants, Spinosad alone or in combination with botanical capsules could efficiently suppress adult emergence by $92.2 \%$ and $80.4 \%$ in comparison to the other plants. These results highlight the high efficacy of Spinosad treatments in reducing fungus gnats.
\end{abstract}

Keywords: fungus gnats, Metarhizium brunneum, Spinosad, oviposition, basil, parsley, poinsettia, adult emergence 


\section{Introduction}

In greenhouse production systems, fungus gnats of the genus Bradysia spp. (Diptera: Sciaridae) are major insect pests (Hamlen \& Mead, 1979). Fungus gnats of the genus Bradysia spp. are the most common species of the family Sciaridae (Menzel et al., 2003, Mohrig et al., 2013). Adults fly near the surface of the growing medium and only cause minimal plant damage (Cloyd, 2000); they are typically reported as aphagous (Kennedy, 1976). Mohrig \& Menzel (2010) described that fungus gnat adults are able to feed on pollen or carbohydrate-rich sources. Females Bradysia spp. are also capable to transmit some fungal pathogens e.g. Verticillium dahliae, Fusarium acuminatum or Thielaviopsis basicola, respectively (El-Hamalawi, 2008) and lay 100 to 200 eggs under debris or into the crevices of the growing medium (Cloyd, 2008). Following the development of the eggs, the emerging larvae feed on organic matter and fungi in the growing media. Fungus gnat larvae are mainly causing direct plant damage on the roots tissues and stem systems of healthy plants by disrupting the ability of plants to uptake water and nutrients, and are interacting with plant pathogens as disease vectors including Pythium spp., Fusarium spp., and Verticillium spp (Elmer 2008, Braun et al., 2012). A study of Jagdale et al. (2004) indicated that fungus gnat larvae are causing a serious damage to the roots of various ornamental plants in greenhouses and nurseries including Capsicum, Cyclamen, Poinsettia (Euphorbia pulcherrima), Geranium, transvaal daisy (Gerbera jamesonii) and Impatiens. In general, young plants or seedlings are more susceptible to injury from fungus gnat larval feeding as compared to mature plants (Gauthier, 1989, Chambers et al., 1993).

The control of fungus gnats can usually be achieved by insecticide applications and biological control agents including entomopathogenic nematodes (EPNs) (Gouge \& Hague, 1995, Jagdale et al., 2004, Katumanyane et al., 2018), predatory mites (Chambers et al., 1993, Jess \& Bingham, 2004, Freire et al., 2007) and biopesticide Bacillus thuringiensis (Osborne et al., 1985, Cloyd \& Dickinson, 2006). However, the use of synthetic insecticides for fungus gnats control is widely restricted due to the development of the insect resistance, and environmental and human health concerns (Bartlett \& Keil, 1997, Bethke \& Dreistadt, 2013).

It is thus necessary to exploit an alternative method for fungus gnat control, emphasizing biological control agents through microbial microorganisms such as entomopathogenic fungi (EPF). Metarhizium anisopliae (Hypocreals: Clavicipitaceae) is one of the most common entomopathogenic fungus (EPF) against a wide range of insects including Diptera e.g. Culicidae (Popko et al., 2018), fruit flies (Tephritidae) (Yousef et al., 2013) or Sciaridae (Tavoosi Ajvad et al., 
2019). This fungal species is able to express virulence against immature or adults of insects, but it is also able to secrete toxins which can be toxic through injection, ingestion and topical application (Golo et al., 2014). M. anisopliae has been recently reported for the control of fungus gnats Lycoriella auripilla larvae (Diptera: Sciaridae) in mushroom production (Tavoosi Ajvad et al., 2019). Additionally, in our previous studies (Chapter 3), M. brunneum has been found to be effective against the larvae of fungus gnat species B. impatiens. Andreadis et al. (2016) reported the EPF Beauveria bassiana as a biological control agent of fungus gnats of the species $L$. ingenue in mushroom production systems.

Spinosad, a natural insecticides derived from the Actinomycete bacteria Saccharopolyspora spinosa (Mertz \& Yao, 1990) is a biorational product and developed for the control of insects pests in agriculture. This product is a combination of two neurotoxic compounds: spinosyins $A$ and $D$ and have low toxicity on non-target organisms and mammals with low persistence in the environment (Bret et al., 1997, Thompson et al., 2000). The mode of action of this bioinsecticide targets nicotinic acetylcholine and gamma-amino butyric acid (GABA) receptor functions, which can contribute to disrupt the insect nerve system (Salgado, 1998). The toxicity of Spinosad has been reported against variety of insect pests in Diptera e.g. Aedes aegypti (Diptera: Culicidae) (Perez et al., 2007), phorid flies Megaselia halterata (Diptera: Phoridae) (Erler et al., 2009) and other mosquito species (Darriet et al., 2005, Romi et al., 2006). This bioinsecticide has, however, so far not been evaluated against fungus gnats (Diptera: Sciaridae). The effectiveness of this bioinsecticide may help to develop a more efficient management program for controlling fungus gnats.

The present study builds upon the results of a previous study (Chapter 3), which reports a higher reduction of adult emergence from substrates treated with a combination of a biopesticide, specifically Spinosad, and a botanical compound (Minx) in an "attract and kill" strategy. Using this strategy, insects are attracted towards an attracting source, and thus they are more subjected to the killing agents (El-Sayed et al., 2009). There have been some previous reports on the combined application of two biological agents against fungus gnats, reporting an increased efficacy in fungus gnat control. According to Tavoosi Ajvad et al. (2019), the combined application of EPF $M$. anisopliae and a predatory mite Gaeolaelaps aculeifer enhanced the control of fungus gnat larvae L. auripilla in mushroom productions. Acharya et al. (2019) also demonstrated an improved efficacy control of fungus gnats $B$. impatiens by the combination of an entomopathogenic nematode EPN (Heterorhabditis indica) and the predatory mite (Stratiolaelaps scimitus). The 
practical application of an EPN (Steinernema feltiae) and an insecticide (imidacloprid) provided an effective and environmental safety strategy for controlling B. odoriphaga in chive production (Yan et al., 2019).

In recent years, biological products gained increased attention for the control of fungus gnats in different ornamental and herb production systems. Most studies have addressed the use of biological products e.g. EPNs to control fungus gnats Bradysia spp. in various crops including fuchsias (Gouge \& Hague, 1995), poinsettia, Euphorbia pulcherrima (Harris et al., 1995, Jagdale et al., 2004, Jagdale et al., 2007), New Guinea impatiens, Impatiens hawkeri (Jagdale et al., 2004), Daisy gerbera plants [Gerbera sp. L. (Asterales: Asteraceae)] (San-Blas et al., 2017), Chinese chive, Allium tuberosum (Ma et al., 2013a) and oyster mushroom, Pleurotus ostreatus (Kim et al., 2001).

Herbs are generally considered as a minor or special group of crops; hence there is a limited number of pest control products available for controlling pests in herbs (Cox \& Craker, 1994b). Among the registered products, Spintor ${ }^{\circledR}$ (Spinosad active ingredient) was reported as a safe bioinsecticide for pest control in herbs such as fennel and parsley, vegetables and crops (Eger \& Lindenberg, 1998). However, the effectiveness of bioinsecticides (e.g. EPF M. brunneum and Spinosad) has not yet been studied for the control of fungus gnats $B$. impatiens in plant production systems such as basil, parsley and poinsettia. In the present study, we assessed the effectiveness of the combined application of a biopesticide and a botanical compound to control the fungus gnat species B. impatiens using three different plant species: basil (Ocimum basilicum L.), parsley (Petroselinum crispum Mill.) and poinsettia (E. pulcherrima Willd.ex Koltzch) under greenhouse conditions.

In the present study, a botanical capsule (Minx) was used as an attractive agent. This capsule, containing mint oil was formulated in a slow release capsule. The component of this capsule was encapsulated in sodium $(\mathrm{Na})$-alginate solution as a carrier material and stabilized by calcium alginate matrix as hydrogel as described previously (Chapter 3).

The objectives of this study were: (1) to determine the ovipositional preference of fungus gnat females on different host plant species (basil, parsley and poinsettia) soils treated with biopesticides ( $M$. brunneum and Spinosad) individually or in combination with the Minx capsules under greenhouse conditions, (2) to evaluate the efficacy of these biopesticides alone or in combination with the Minx capsules aimed at reducing adult fungus gnat numbers and (3) to assess the effectiveness of the combined application of these biopesticides with the different rates of the Minx capsules on the control efficiency of fungus gnat using poinsettia plants. 


\section{Materials and methods}

Insect rearing

Laboratory colonies of the fungus gnat species $B$. impatiens were obtained from a permanent colony maintained in Agricultural Entomology section, Crop Science Department, Georg-August Universität Göttingen, Germany. Fungus gnat adults were reared in plastic pots $(11 \mathrm{~cm} \times 11 \mathrm{~cm} \times$ $12 \mathrm{~cm}$ ) filled with $350 \mathrm{gr}$ of a favorable growing medium, $6 \mathrm{gr}$ horn shives (Klasmann-Deilmann GmbH; Geeste, Germany), oatmeal (3 gr) and a shredded potato (Cloyd \& Zaborski, 2004) under a photoperiod of $16: 8 \mathrm{~h}(\mathrm{~L}: \mathrm{D})$ and a temperature of $23 \pm 2^{\circ} \mathrm{C}$. For each experiment, adults were collected from the permanent colonies and were released into a small rectangle container (Nette Papier GmbH, Göttingen, Germany) containing moist growing medium as described above (Klasmann-Deilmann GmbH; Geeste, Germany) to allow oviposition. 48h after oviposition, the container including the eggs were maintained into a room at $23 \pm 2^{\circ} \mathrm{C}$ to allow development to fungus gnat adults. The newly emerged adults were collected from the container via an aspirator and used in the bioassays.

\section{Sources of plant material}

Basil: Sweet basil (Ocimum basilicum) seeds (Bio-Saatgut Basilikum, Kiepenkerl ${ }^{\circledR}$, Bruno Nebelung $\mathrm{GmbH}$, Everswinkel, Germany) were sown in a rectangle container filled with a moist substrate (Fruhstorfer Erde Typ P25; Hawita Gruppe GmbH, Vechta, Germany), sand and compost (2:1:1), and covered with a thin layer of substrate after sowing. A seedling of basil was transferred after 15 days sowing into a plastic cup (300 ml, Nette Papier $\mathrm{GmbH}$, Göttingen, Germany) filled with $250 \mathrm{ml}$ substrate (Fruhstorfer Erde Typ P 25; Hawita Gruppe GmbH, Vechta, Germany). These plants were maintained in a rearing tent (BugDorm-2 Insect Tents, MegaView Science Co. Ltd.; Taichung, Taiwan) to prevent the plants from unintended fungus gnat contamination in the greenhouse at $15^{\circ} \mathrm{C} \pm 2$ (April - May 2019). Plants were irrigated with water as needed or a fertilizer solution (10 $\mathrm{ml}$ per cup; (15-10-15(+2); Hakaphos ${ }^{\circledR}$ Blau) on a weekly schedule from the surface. Basil plants at a height of $20 \mathrm{~cm}$ were used for our experiments.

Parsley: Seeds of parsley (Petroselinum crispum) variety neapolitanum (Petersilie Gignate di Napoli, Kiepenkerl ${ }^{\circledR}$, Bruno Nebelung $\mathrm{GmbH}$, Everswinkel, Germany) were planted in a container including moist substrate (Fruhstorfer Erde Typ P 25; Hawita Gruppe GmbH, Vechta, Germany), sand and compost (2:1:1) to sprout as described above for basil plants. About 3-week-old seedling of parsley was transplanted into a plastic cup filled with $250 \mathrm{ml}$ substrate (Fruhstorfer Erde Typ 
P25; Hawita Gruppe $\mathrm{GmbH}$, Vechta, Germany) and placed in the greenhouse at $24{ }^{\circ} \mathrm{C} \pm 2$ (August September 2019). Plants were kept in a rearing tent and irrigated as described for basil plants. Parsley plants were used with the same height as the basil plant.

Poinsettia: poinsettia plants (Euphorbia pulcherrima) were obtained from a horticulture grower (Gartenbau Heinz von Danwitz GmbH, Tönisvorst, Germany) and maintained in the greenhouse until use (October - November 2019; December - January 2020). Poinsettia plants (20-25 leaves) were watered as needed and plants with $15 \mathrm{~cm}$ height were used for our experiments.

\section{Treatments}

"Fungal preparation": A fungal isolate of M. brunneum ( $\mathrm{Cb} 15)$ was obtained from the Agricultural Entomology section, Georg-August Universität Göttingen, Germany. To prepare the conidial suspension for the experiments, a fungal culture of the selected isolate was subcultured on potato extract glucose agar (PDA; Carl Roth ${ }^{\oplus}$, Germany) grown for ca.3 weeks in a climate chamber (Biologischer Klimaschrank WB 750, Mytron Bio- und Solartechnik GmbH, Heilbad Heiligenstadt, Germany) at $25 \pm 1^{\circ} \mathrm{C}$ and $60 \pm 5 \% \mathrm{RH}$ in darkness. The spore suspension was prepared by scraping conidia from Petri plates into a $50 \mathrm{ml}$ sterile demineralized water plus Tween 80 (0.1\% v/v; Carl Roth $^{\circledR}$, Germany). This initial conidial suspension was filtered through a fine mesh to remove mycelium and agar residues. The spore suspension was verified with light microscopy (200X magnification, BH2-HLSH, Olympus Corporation, Tokyo, Japan). The conidial suspension used for the bioassay was adjusted by diluting the conidia with demineralized water to a final concentration of $1 \times 10^{7}$ spores/ml. The number of spores was calculated using a Neubauer Hemocytometer (0.1 mm depth) (Thoma CE, Paul Marienfeld GmbH \& Co.KG, Lauda-Königshofen, Germany).

"Attract": Botanical capsules (Minx) were obtained from Katz Biotech AG; Baruth, Germany and stored in a cooling chamber $\left(6^{\circ} \mathrm{C}\right)$ until use. Minx capsules consisted of vegetable oil (approximately 50\% dry matter), ( $\mathrm{Na}$-alginate solution, silica gel, mint oil and water (5\% in dry matter). These components were encapsulated in a ( $\mathrm{Na}$ )-alginate solution and formulated in a slow release capsule as described in our previous study (Chapter 3). A solid form of this capsule was used for our bioassays (J. Rademacher, personal communication, Katz Biotech AG; Baruth, Germany). Minx capsules with a high rate of mint oil (1\% and $10 \%$ mint oil) were recommended by J. Rademacher (Katz Biotech AG; Baruth, Germany) for this study. The same amount of Minx capsules ( $2 \mathrm{mg}$ ) was used based on our previous experiments (Chapter 3 ). 


\section{Experimental design}

Experiment 1: combined application of a biopesticide and a botanical compound to control fungus gnats in basil plants

We assessed the effectiveness of two biopesticides ( $M$. brunneum and Spinosad) individually or in combination with the botanical capsule (Minx) against fungus gnats using basil plants in the greenhouse. In this trial, the surface of the substrate was covered by a fine mesh ( $9 \mathrm{~cm}$ diameter). Prior to treating the substrate, a small portion (2 g) of the substrate (Fruhstorfer Erde Typ P 25; Hawita Gruppe $\mathrm{GmbH}$, Vechta, Germany) supplemented with three to four pieces of oatmeal were added to cover the surface of the mesh in each experimental cup to provide an appropriate site for fungus gnat females to lay eggs. This mesh assisted in counting the numbers of eggs laid by the females. Minx capsules containing $1 \%$ mint oil were added at a rate of $2 \mathrm{mg}$ to the substrate above the mesh. $10 \mathrm{ml}$ of $M$. brunneum isolate $\mathrm{Cb} 15$ or Spinosad (Spintor ${ }^{\circledR}: 480 \mathrm{~g}$ Spinosad active ingredient per liter, Dow AgroSciences Kings Lynn Norfolk, United Kingdom) were drench-applied at a rate of $1 \times 10^{7} \mathrm{spore} / \mathrm{ml}$ or $1 \%$ into the substrate. The treatments were homogenously incorporated in the substrate above the mesh. The concentration of Spinosad was chosen based on our preliminary dose-response bioassays using third instar fungus gnat larvae with a concentration of $1 \%$ as the minimum concentration. Immediately after treatment, eighty fungus gnat adults (mixture of females and males; sex ratio about 50\%) were released into each experimental chamber with potted plants and the soils drenched with either the fungal suspension or Spinosad individually or in combination with the botanical capsules in the greenhouse. $48 \mathrm{~h}$ after exposure, the numbers of eggs laid by females was counted under a binocular microscope (ZEISS, Carl Zeiss Microscopy GmbH, Jena, Germany). After counting, Basil plants were cut and the eggs were returned into the plastic cups. Cups were maintained in a climate chamber at $25 \pm 1^{\circ} \mathrm{C}$ and $65 \pm 5 \% \mathrm{RH}$ with a photoperiod: 16:8 h (Biologischer Klimaschrank WB 750, Mytron Bio- und Solartechnik $\mathrm{GmbH}$, Heilbad Heiligenstadt, Germany) and allowed to complete their life cycle to adults. Each cup was closed with a lid. The number of the emerging adults was monitored via a yellow sticky trap $(2.5 \times 2.5 \mathrm{~cm})$ attached at the top of each plastic cup. Control cups received sterilized water with the same volume as the biopesticide treatment. Three weeks after treatment, the numbers of emerged adults in each cup were recorded. The trial was terminated when no new adults were counted within a period of 4 days. This bioassay was conducted in the experimental chamber with four replicates and each chamber consisted of two plants for each treatment. 
Experiment 2: combined application of a biopesticide and a botanical compound to control fungus gnats in parsley plants

In this trial, the efficacy of two biopesticides ( $M$. brunneum isolate $\mathrm{Cb} 15$ and Spinosad) individually or in combination with the botanical capsule Minx to control fungus gnats in parsley plants were tested in the greenhouse. We followed the same treatment application procedures and experimental management as described above in experiment 1 for the basil plants. $10 \mathrm{ml}$ of the fungal suspensions or Spinosad were added at the same rate mentioned above to the substrate on the mesh. Eighty fungus gnat adults were transferred into each experimental chamber with potted plants and the substrates treated with the fungal suspension or Spinosad individually or in combination with the botanical capsules containing $1 \%$ mint oil. This experiment was set-up in the greenhouse. $48 \mathrm{~h}$ post treatments (HPT), the number of eggs laid by females were counted. Plants were cut and the eggs were returned into the plastic cups. All the plastic cups containing eggs laid by females were closed with a lid and were transplanted into a climatic chamber (air temperature and humidity: $25 \pm 1^{\circ} \mathrm{C}, 65 \pm 5 \%$; photoperiod: 16:8 h). 22 days after treatment, the number of adults emerged were recorded using yellow sticky cards as mentioned above. The control cups were treated with sterilized water. A total of four experimental chambers were designed, each having two 2 plants for each treatment.

Experiment 3: combined application of a biopesticide and a botanical compound to control fungus gnats in poinsettia plants

We studied the control of fungus gnats in poinsettia plant soils drenched with $M$. brunneum isolate $\mathrm{Cb} 15$ or Spinosad individually or in combination with the botanical capsule (Minx). All procedures were the same as described in pervious experiments except that botanical capsules including $10 \%$ mint oil were used. Due to the strong volatiles derived from Minx capsules containing $10 \%$ mint oil, these Minx capsules were selected for this trail. The substrate supplemented with oat meal was added to cover the surface of the mesh (10 cm diameter). The botanical capsules containing $10 \%$ mint oil (at a rate of $2 \mathrm{mg}$ ) were buried in the substrate above the mesh. $10 \mathrm{ml}$ of the fungal suspension of M. brunneum or Spinosad were added at a rate of $1 \times$ $10^{7} \mathrm{spore} / \mathrm{ml}$ or $1 \%$ to the substrate on the mesh and then homogenously incorporated within the substrate as described above. Following drench application, eighty fungus gnat adults (mixture of females and males; sex ratio about 50\%) were released into each experimental chamber to oviposit on the mesh. $48 \mathrm{~h}$ post treatment, the number of eggs laid by females was counted under 
a binocular microscope. Poinsettia plants were cut and the eggs were returned into the plastic cups. Experimental cups including eggs laid by females were closed with a lid $(11 \mathrm{~cm}$ diameter, Nette Papier $\mathrm{GmbH}$, Göttingen, Germany) and placed in a climatic chamber at $25 \pm 1^{\circ} \mathrm{C}$ and $65 \pm$ 5\% RH with a photoperiod: 16:8 h. After 22 days, the next generation of fungus gnat adults began to emerge and the total number of adults emerged from each experimental cup via a yellow sticky trap $(2.5 \times 2.5 \mathrm{~cm})$ attached at the top of each plastic cup was counted. Sterilized water was used as the control treatment. A total of four replications (= experimental chambers) were set up, each experimental chamber including two potted plants for each treatment.

Experiment 4: combined application of a biopesticide and a botanical compound at different rates to control fungus gnats in poinsettia plants

We assessed the effectiveness of biopesticides $M$. brunneum $\mathrm{Cb} 15$ or Spinosad individually or in combination with the botanical capsule Minx at different rates against fungus gnats in poinsettia plants in the greenhouse. The experimental procedures and methods used were the same as described in experiment 3. Each pot was covered with a fine mesh with $10 \mathrm{~cm}$ diameter and the substrate (2 g) (Fruhstorfer Erde Typ P 25; Hawita Gruppe GmbH, Vechta, Germany), supplemented with three to four pieces of oatmeal were added to cover the surface of the mesh as described in the previous trials. Minx capsules containing $10 \% \mathrm{mint}$ oil at different rates $(1,5$ and 10 capsules) were added individually into each potted plants to the substrates on the mesh. $10 \mathrm{ml}$ of the fungal suspensions or Spinosad were drench-applied at a rate of $1 \times 10^{7}$ spores $/ \mathrm{ml}$ or $1 \%$ to the substrate above the mesh. The treatments were homogenously incorporated within the substrate. Following treatments, eighty fungus gnat adults (mixture of females and males; sex ratio about 50\%) were released into each experimental chamber consisting of potted plants and the substrates drenched with the biopesticide treatments alone or in combination with the Minx capsules. $48 \mathrm{~h}$ after treatment, the number of eggs laid by the fungus gnat females were counted. Plants were cut and the eggs were returned into the pots. The pots including eggs on the mesh were closed with a lid as described above and were transferred into a climatic chamber the same conditions as mentioned in experiment 3. After 3 weeks, the emergence of fungus gnat adults was monitored using yellow sticky card fixed at the top to trap the emerging adults. This bioassay was conducted in the experimental chamber with eight replicates, each experimental chamber including one plant for each treatment. 


\section{Data analysis}

All Statistical analyses was run with R version 3.5.3 and graphs were also created with R. Data were analyzed using the general linear model (GLM) with quasipoisson distribution, and differences between treatments were tested with a Tukey HSD test at $5 \%$ level of significance. The number of fungus gnat adults which emerged from each treatment were calculated as percentages and analyzed as independent variables. All data were first checked for normality and error variance for homoscedasticity. Fungus gnat females did not lay eggs on some host plants. These data were not considered in the analyses.

\section{Results}

Experiment 1: combined application of a biopesticide and a botanical compound to control fungus gnats in basil plants

Fungus gnat females laid more eggs, although not significantly, in basil plants soils treated with the botanical capsules Minx $(65.28 \pm 12.79$; mean \pm SE) or the control treatment $(60.25 \pm 16.61$; mean $\pm S E)$ compared to the fungal or Spinosad treatments $\left(F_{5,34}=7.69 ; p<0.001 ;\right.$ Fig. 1$)$. There were highly significant differences among the basil plant soils treated with the fungal suspension of $M$. brunneum and Spinosad individually or in combination with the botanical capsules in terms of number of eggs laid $\left(F_{3,21}=12.93, p<0.05\right)$. Fungus gnat females exhibited an ovipositional preference on the basil plant substrates treated with $M$. brunneum alone (53 \pm 10.97 ) or in combination with the botanical capsules $(49.45 \pm 13.24)$ as compared to Spinosad treatments. The lowest number of eggs laid by the females was found on the basil plant soils treated with Spinosad alone $(2 \pm 0.54)$ or in combination with the botanical capsule Minx $(1.5 \pm 0.5)$, with no significant differences between these two treatments $\left(F_{1,7}=0.43, p=0.532\right)$.

In the subsequent bioassay, the emergence of fungus gnat adults from eggs revealed that $71.88 \%$ and $58.9 \%$ of eggs laid in basil plant soils in the control and the botanical capsule Minx could successfully develop to adults. The number of eggs laid by the females in basil plant substrates was negatively affected by the fungal suspension or Spinosad treatments, resulting in a reduction of adult emergence as compared to the control or the botanical capsule alone treatments $\left(F_{5,34}=1.37 ; p=0.25\right.$; Fig. 2). The number of adult emergence from eggs laid in basil plant soils treated with $M$. brunneum varied from $43.41 \%(M b+M i n x)$ to $46.22 \%$ M. brunneum alone $(M b)$, with no significant differences between two treatments $\left(F_{1,14}=0.028 ; p=0.86\right)$. Additionally, Spinosad treatments reduced, although not significantly, adult emergence; only $25 \%$ 
of eggs laid in Spinosad alone or in combination with the Minx capsules finalized their life cycle to adults.

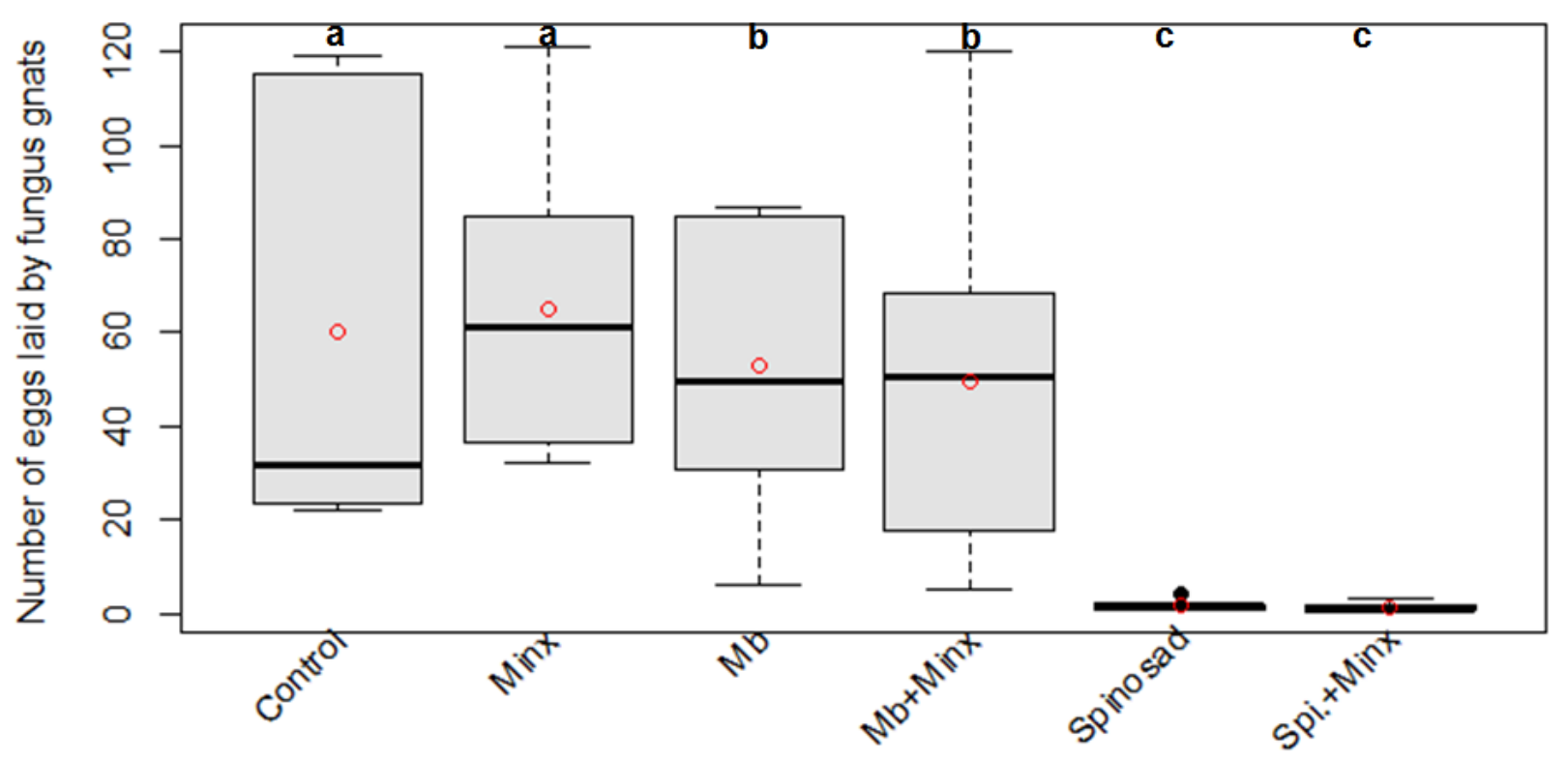

Treatments

Fig. 1: Oviposition of fungus gnat females in basil plant soils treated with the fungal suspension $M$. brunneum (Mb) and Spinosad (Spi.) individually or in combination with the botanical capsule (Minx) vs. control treatments; different letters indicate significant differences between treatments after Tukey HSD test $(p<0.05)\left(F_{5,34}=7.69\right)$.

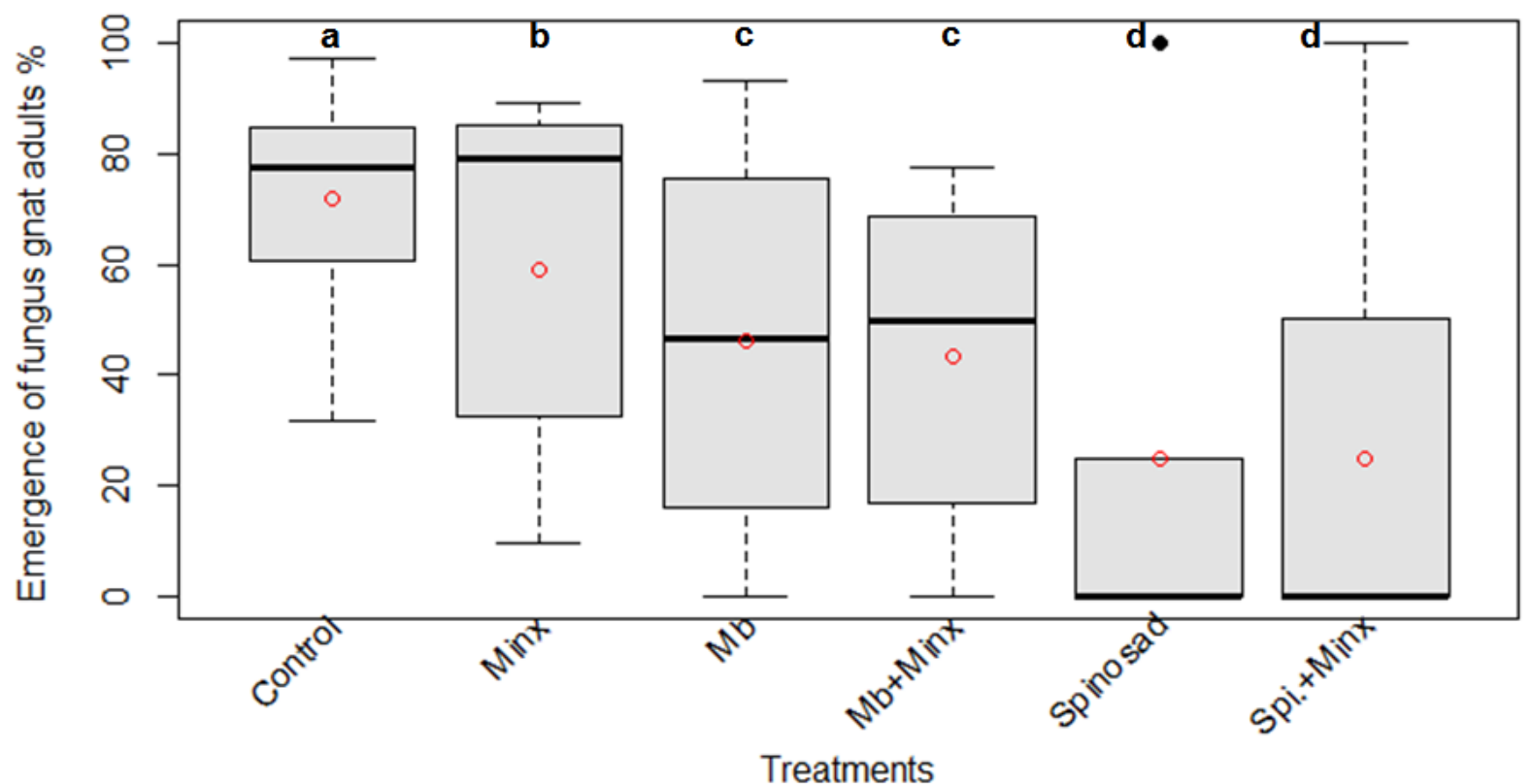

Fig. 2: Emergence of fungus gnat adults (\%) from eggs laid by the females in basil plant soils treated with the fungal suspension M. brunneum (Mb) and Spinosad (Spi.) individually or in combination with the botanical capsule (Minx) vs. control treatments; different letters indicate significant differences between treatments after Tukey HSD test $(p<0.05)\left(F_{5,34}=1.37\right)$. 
Experiment 2: combined application of a biopesticide and a botanical compound to control fungus gnats in parsley plants

Fungus gnat females showed a strong ovipositional preference for parsley plant substrates treated with Spinosad individually $(124 \pm 35.62$; mean \pm SE) or in combination with botanical capsules $(88.42 \pm 16.04 ;$ mean $\pm S E)$ compared to the fungal and the control treatments $\left(F_{5,40}=1.32 ; p=\right.$ 0.28; Fig. 3). Additionally, using parsley plant, fungus gnat females laid more eggs, although not significantly, in the substrates containing the botanical capsules $(77.75 \pm 8.92$; mean $\pm \mathrm{SE})$ or control substrate $(78.37 \pm 25.66$; mean $\pm S E$ ) as compared to the substrate treated with the fungal suspension of $M$. brunneum alone $(62 \pm 13.87)$ or in combination with the Minx capsules (63.12 \pm 8.75) $\left(F_{3,28}=0.33 ; p=0.81\right)$.

In the following experiment, the emergence of adults from eggs laid by the females in the parsley plants showed that the lowest number of adults hatched from Spinosad treatments individually (25.92 \%) or in combination with the botanical capsule $(28.34 \%)$ as compared to the fungal or the control treatments $\left(F_{5,40}=5.154 ; p<0.001 ;\right.$ Fig. 4). In addition, eggs laid by fungus gnat females in parsley soils treated with the fungal suspension of $M$. brunneum alone or in combination with the botanical capsule could not completely develop to adults; resulting in a significant reduction of the adult emergence, $48.93 \%$ and $42.72 \%$, respectively, in comparison to the control or the botanical capsule treatments. Furthermore, the highest percentage of fungus gnat adults were recorded from the substrates treated with Minx $(51.46 \%)$ and the control treatments $(57.42 \%)$, with no significant differences between the these treatments $\left(F_{1,14}=0.51 ; p\right.$ $=0.48)$. 


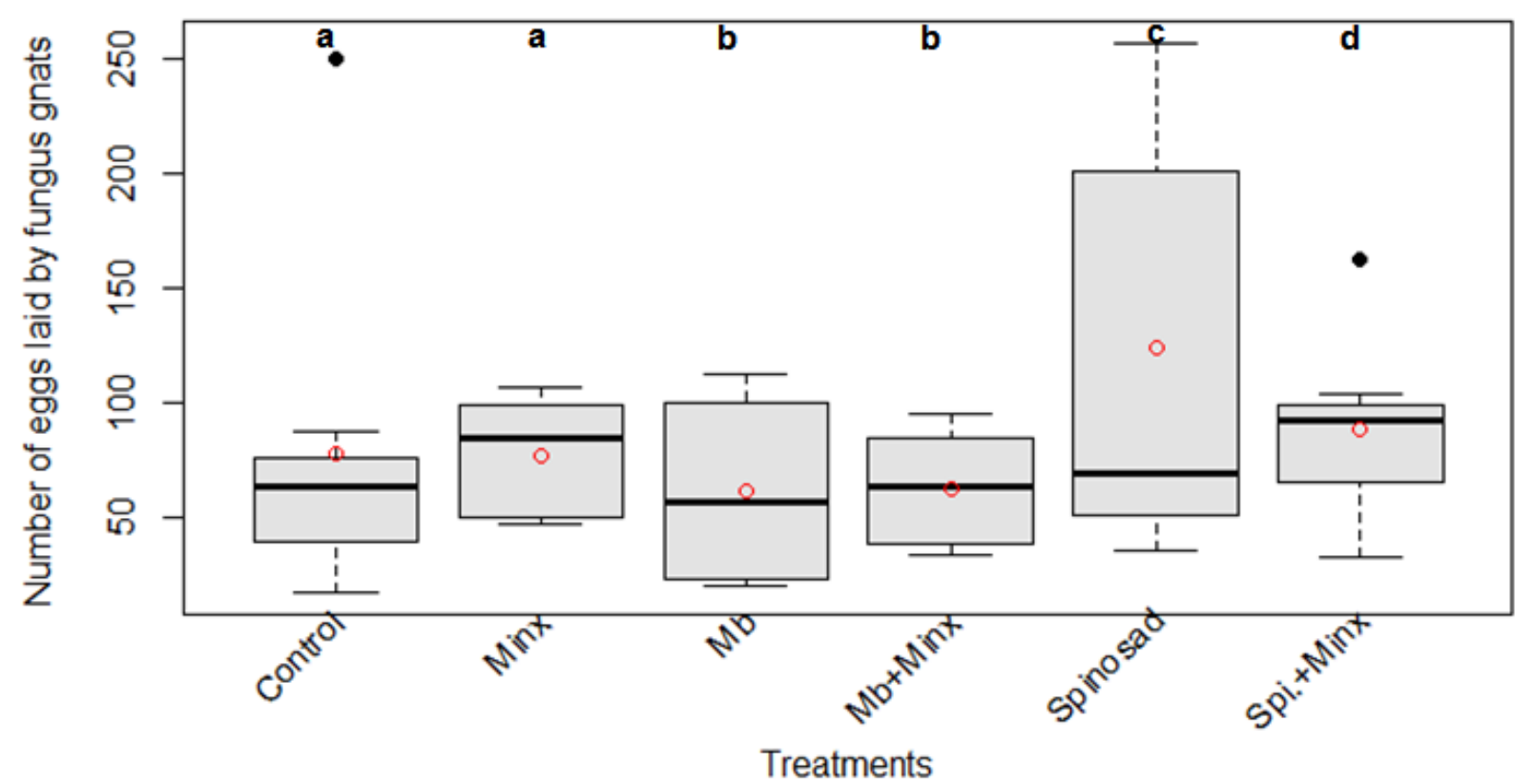

Fig. 3: Number of eggs laid by the fungus gnat females on parsley plant soils treated with the fungal suspension M. brunneum (Mb) and Spinosad (Spi.) individually or in combination with the botanical capsule (Minx) vs. control treatments; different letters indicate significant differences between treatments after Tukey HSD test $(p<0.05)\left(F_{5,40}=1.32\right)$.

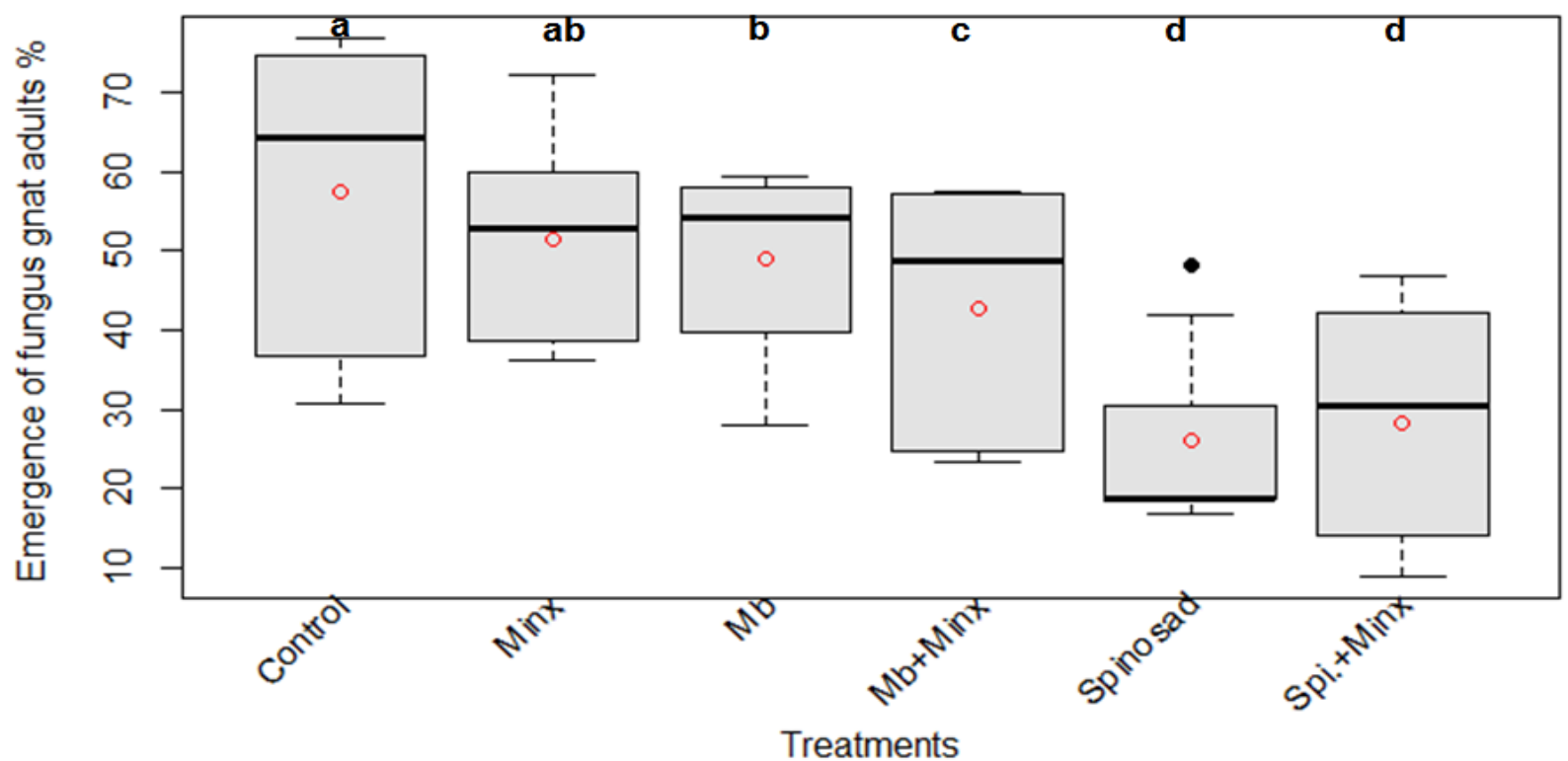

Fig. 4: Emergence of fungus gnat adults (\%) from eggs laid by the females in parsley plant soils treated with the fungal suspension M. brunneum (Mb) and Spinosad (Spi.) individually or in combination with the botanical capsule (Minx) vs. control treatments; different letters indicate significant differences between treatments after Tukey HSD test $(p<0.05)\left(F_{5,40}=5.154\right)$. 
Experiment 3: combined application of a biopesticide and a botanical compound to control fungus gnats in poinsettia plants

Poinsettia plant substrates treated with Spinosad individually or in combination with the botanical capsule received the highest number of eggs laid by the females as compared to the fungal or control treatments $\left(F_{5,40}=7.47 ; p<0.001 ;\right.$ Fig. 5$)$. High numbers of eggs were laid by fungus gnat females in Spinosad-treated plant substrates when applied alone or in combination with the botanical capsules (34.83 \pm 9.17 and $21.87 \pm 3.45$; mean $\pm \mathrm{SE}$, respectively). In contrast, low numbers of eggs laid by the females were recorded in poinsettia soils treated with fungal suspension alone $(10.87 \pm 1.72$; mean $\pm \mathrm{SE}$ ) or in combination with the botanical capsule (11 \pm 1.43; mean \pm SE). Additionally, a high attractiveness of fungus gnat females for oviposition was found for the control treatments $(18.62 \pm 2.18$; mean $\pm \mathrm{SE})$ as compared to the botanical capsules $\min x(13.12 \pm 1.56 ;$ mean $\pm S E)\left(F_{1,14}=4.2 ; p=0.059\right)$.

In addition, the emergence of fungus gnat adults was significantly reduced in the poinsettia soil treated with the fungal suspension or Spinosad when applied alone or in combination with the botanical capsules in comparison to the botanical capsules or the control treatments $\left(F_{5,40}=8.91\right.$; $p<0.001$; Fig. 6). The emergence of adults were negatively affected by Spinosad treatments; thus, only $7.82 \%$ and $19.65 \%$ of all eggs laid by the females in Spinosad-treated pots individually and in combination with the botanical capsule could develop to adults, with a significant difference between these treatments $\left(F_{1,12}=1.76 ; p=0.2\right)$. Additionally, the emergence of adults from eggs laid in poinsettia soils treated with $M$. brunneum varied from $36.23 \%$ in Mb+ Minx to $51.22 \%$ in M. brunneum alone $(M b)\left(F_{1,14}=2.11 ; p=0.16\right)$. Moreover, the number of fungus gnat adults was recorded from the substrates treated with the botanical capsules and the control was recorded $56.68 \%$ and $61.67 \%$, respectively. 


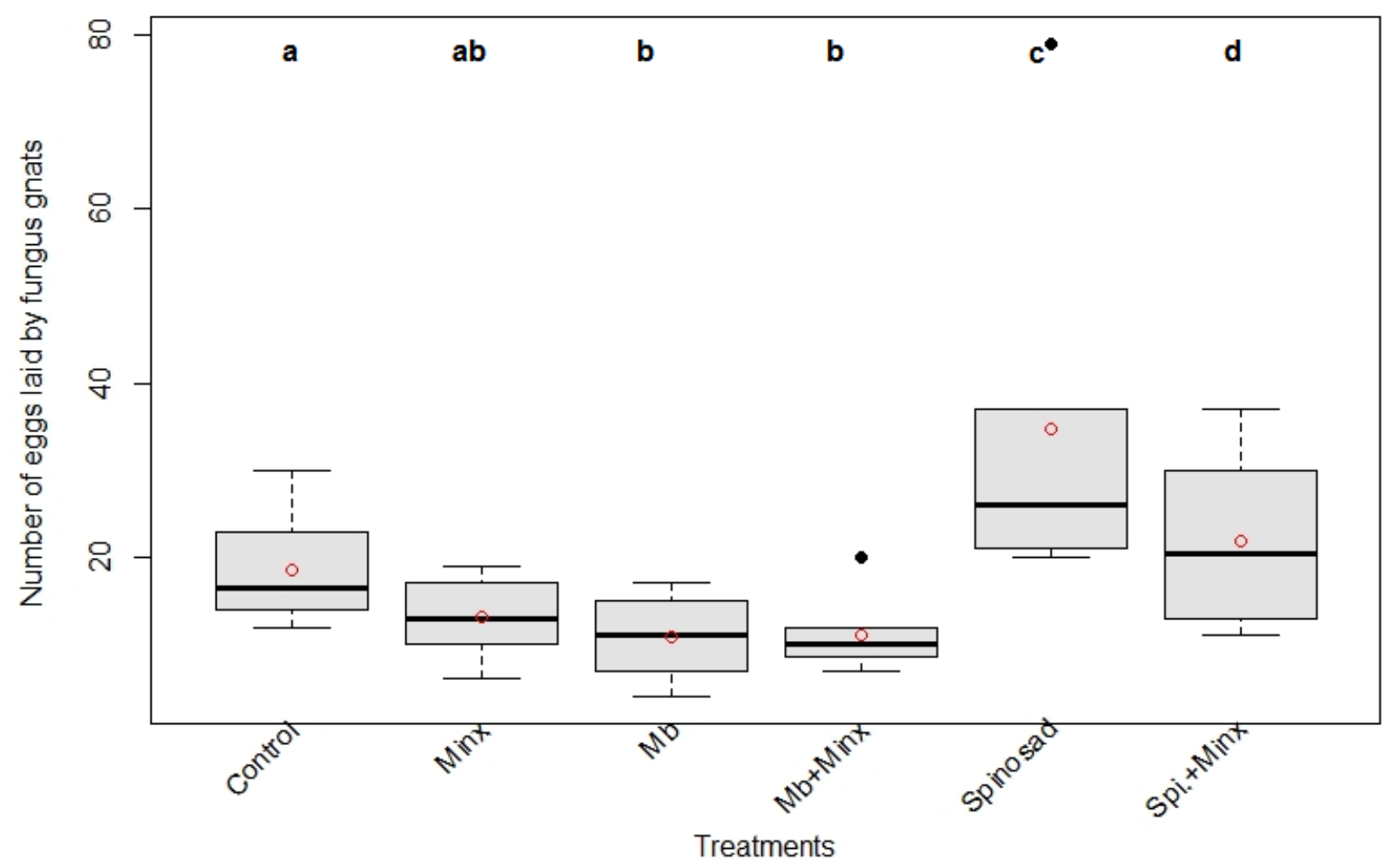

Fig. 5: Number of eggs laid by fungus gnat females in poinsettia plant soils treated with the fungal suspension of M. brunneum (Mb) and Spinosad (Spi.) individually or in combination with the botanical capsule (Minx) vs. control treatments; different letters indicate significant differences between treatments after Tukey HSD test $(p<0.05)\left(F_{5,40}=7.47\right)$.

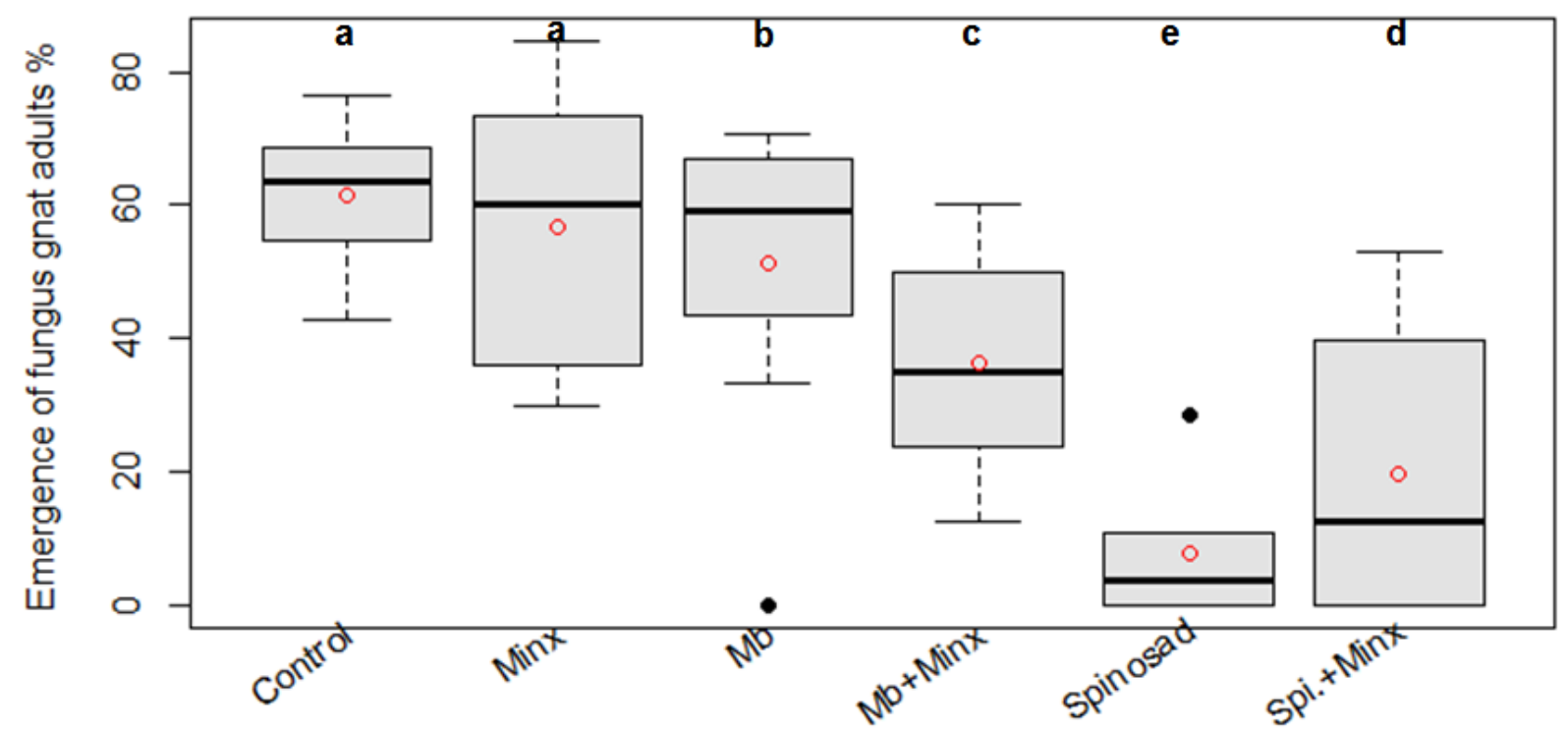

Treatments

Fig. 6: Fungus gnat adult emergence (\%) from eggs laid in poinsettia plant soils treated with the fungal suspension M. brunneum (Mb) and Spinosad (Spi.) individually or in combination with the botanical capsule (Minx) vs. control treatments; different letters indicate significant differences between treatments after Tukey HSD test $(p<0.05)\left(F_{5,40}=8.91\right)$. 
Experiment 4: combined application of a biopesticide and botanical capsules at different rates for fungus gnat control in poinsettia plants

The number of eggs laid by fungus gnat females differed between treatments $\left(F_{11,57}=1.02 ; p=\right.$ 0.43; Fig. 7). Fungus gnat females were attracted to oviposit in the poinsettia plants soil treated with the botanical capsules Minx, ranging from $14.33 \pm 5.45$ in (Minx at a rate of 1 capsule $=$ Minx (1)) to $19 \pm 5.71$ in (Minx at a rate of 5 capsules = Minx (5)) as compared to the control treatment $(8.8 \pm 2.47 ;$ mean $\pm S E)\left(F_{2,16}=1.04 ; p=0.37\right)$, resulting an attractant effect for fungus gnat adults to oviposit. Additionally, Fungus gnats were laid more eggs in Spinosad-treated soils alone or in combination with the botanical capsules Minx at a rate of 1 capsule (Spi. + Minx (1)), $18.20 \pm 10.36$ and $20.80 \pm 10.11$, respectively, as compared to other Spinosad treatments in combination with minx capsules $\left(F_{3,13}=1.07 ; p=0\right.$. 39). However, there were no significant differences between the poinsettia plants soils treated with Spinosad in combination with the Minx capsules at rates of 5 capsules $(S p i .+$ Minx (5)) (8.66 $\pm 4.05 ;$ mean \pm SE) and 10 capsules (Spi. + Minx (10)) $(4.75 \pm 2.42$; mean $\pm S E)$ in terms of number of eggs laid $\left(F_{1,5}=0.76 ; p=0.42\right)$. Furthermore, the number of eggs laid by the females in the poinsettia plant soils treated with $M$. brunneum treatments when applied alone or in combination with the minx capsules ranged from $7.62 \pm 2.04$ (mean $\pm \mathrm{SE}$ ) in $\mathrm{Mb}$ $+\operatorname{Minx}(10)$ to $16.85 \pm 3.65$ (mean $\pm \mathrm{SE})$ in $M b+\operatorname{Minx}(1)\left(F_{3,22}=1.22 ; p=0.32\right)$.

Subsequently, the adult emergence from eggs laid by the females in the poinsettia plant soils varied between treatments $\left(F_{11,57}=0.94 ; p=0.51 ;\right.$ Fig. 8). A higher percentage of adults were emerged from the eggs laid in the plant soils treated with the Minx capsule at a rate of 10 capsules (Minx (10)) (40.37\%) in comparison to the other rates of the botanical capsules (27\% - $28 \%)$. However, there were no significant differences between the different rates of the Minx capsules and the control treatments in terms of adult emergence $\left(F_{3,22}=0.26 ; p=0.84\right)$. Additionally, only $15.25 \%$ of all eggs laid by the females in poinsettia plant soils treated with M. brunneum alone could finalize their life cycle to adults, which was lower than the adult emergence from $M$. brunneum in combination with the botanical capsules Minx. The adult emergence from $M$. brunneum in combination with the botanical capsules Minx varied from $20.31 \%(M b+$ Minx (10)) to $53.86 \%(M b+\operatorname{Minx}(5))\left(F_{3,22}=2.15 ; p=0.12\right)$. Furthermore, the number of adult emergence from the host plant soils treated with Spinosad alone (39.44\%) was higher than the adult emergence from the combination of Spinosad with the botanical capsules $\left(F_{3,13}=1.09 ; p=0.38\right)$. 


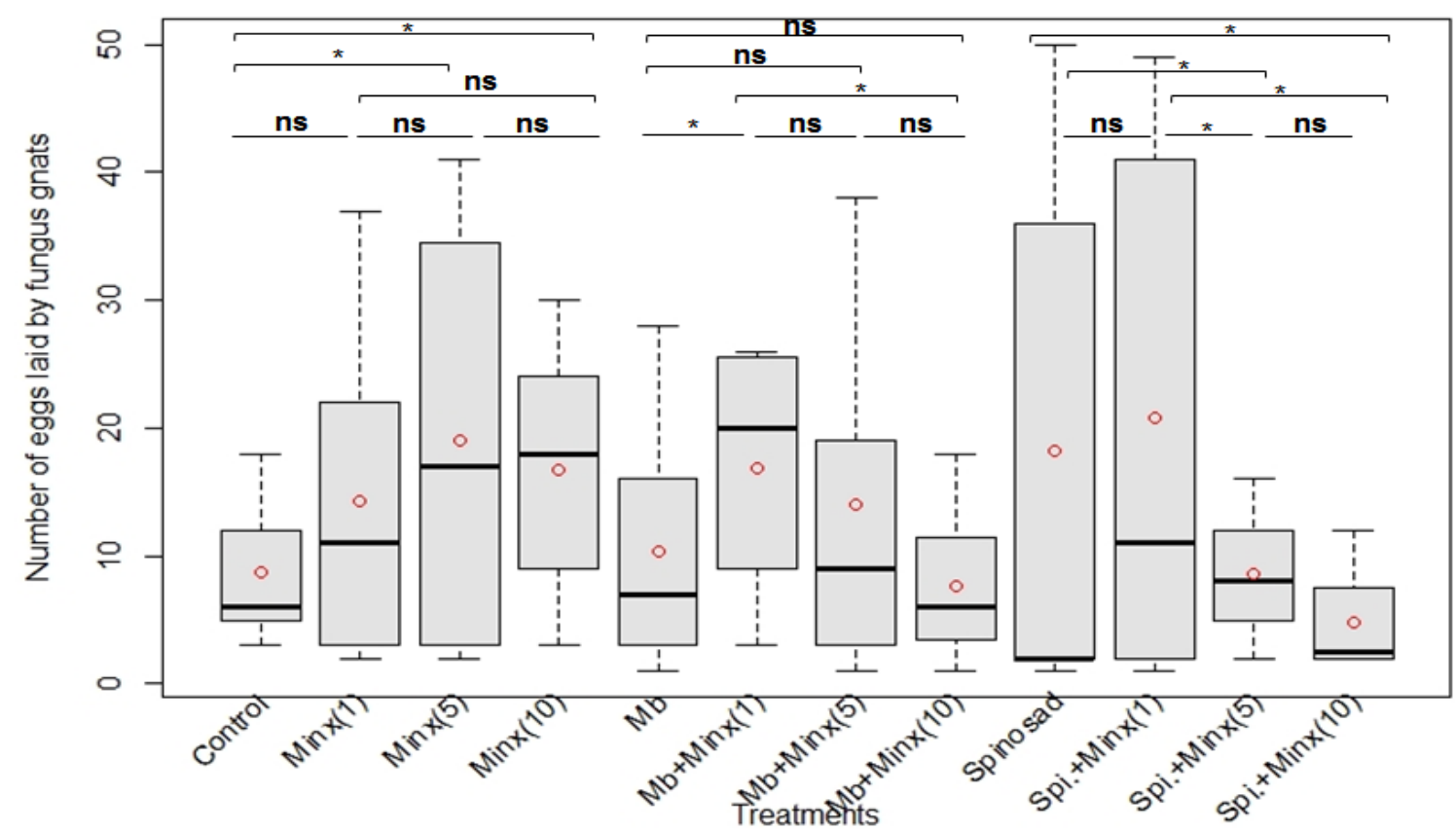

Fig. 7: Oviposition of females on poinsettia plant soils treated with the fungal suspension $M$. brunneum (Mb) and Spinosad (Spi.) individually or in combination with different rates (1, $\mathbf{5}$ and 10 capsules / pot) of the botanical capsule (Minx) vs. control treatments; Asterisks indicate significant differences between treatments in terms of numbers of eggs laid after Tukey HSD test $(p<0.05)(F$ $11,57=1.02)$; ns: not significant.

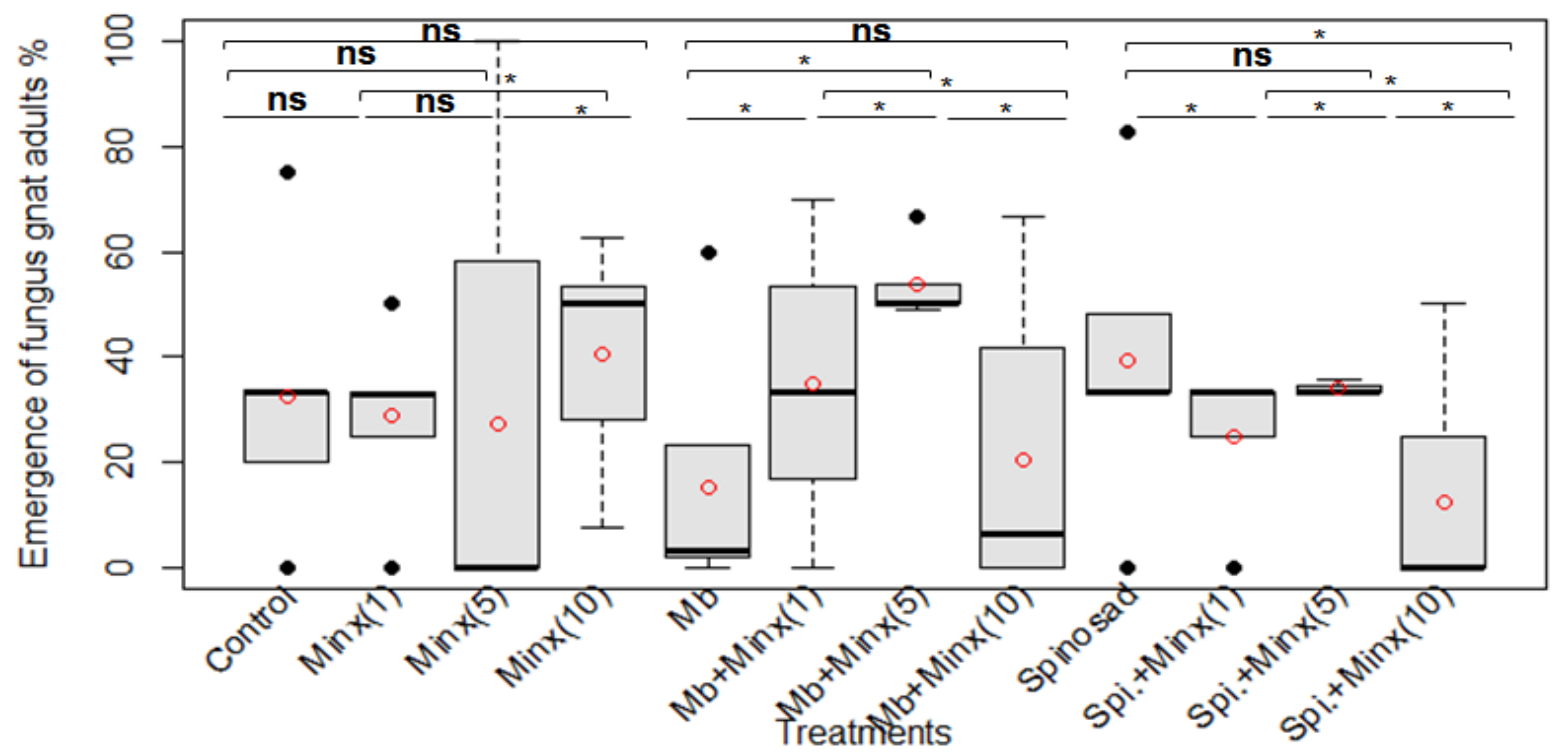

Fig. 8: Emergence of fungus gnat adults (\%) from eggs laid in poinsettia plant soils treated with the fungal suspension M. brunneum (Mb) and Spinosad (Spi.) individually or in combination with different rates (1, 5 and $\mathbf{1 0}$ capsules / pot) of the botanical capsule (Minx) vs. control treatments.; Asterisks indicate significant differences between treatments in terms of adult emergence after Tukey HSD test $(p<0.05)\left(F_{11,57}=0.94\right)$; ns: not significant. 


\section{Discussion}

The present study demonstrated the efficacy of combining a biopesticide and a botanical capsule in an "attract and kill" approach to control fungus gnats on three different host plants (basil, parsley and poinsettia), under greenhouse conditions. Performance of biopesticides in combination with the Minx capsules differed according to the host plants. This study indicated attractiveness of fungus gnat females to lay more eggs in parsley and poinsettia plant soils treated with Spinosad alone or in combination with the botanical capsules Minx as compared to the fungal or the control treatments. Using basil plants, fungus gnat females showed a strong ovipositional preference for the botanical capsules or control treatments. Interestingly, eggs laid by the females in the host plants species treated with Spinosad could not finalize their life cycle to adults, resulting in an efficient reduction of adult emergence (72 \% - $93 \%$ ) when applied alone or in combination with the Minx capsules. We also found that parsley and poinsettia plants soils treated with M. brunneum in combination with the Minx capsules were slightly higher attractive for oviposition of fungus gnats as compared to M. brunneum alone. Despite its attractiveness, the adult emergence from M. brunneum in combination with the botanical capsules was lower than the number of adults emerged from M. brunneum alone. The combination of M. brunneum with the botanical capsules in all of three host plant species tested resulted in a higher efficacy to control fungus gnat larvae as shown by the reduced emergence of adults by $57 \%$ to $64 \%$ in an "attract and kill" approach.

Efficacy of biopesticides individually or in combination with Minx capsules on the control of fungus gnats on three host plants

\section{Spinosad}

Using two host plants species, parsley and poinsettia, fungus gnat females were highly attracted to the potted plants and the soils drenched with Spinosad for oviposition as compared to other treatments. The attractive behavior of fungus gnat females to oviposit in Spinosad treatments might be due to the distinctive aroma of damp earth, which is derived from Actinomycetes. This reason was suggested by Perez et al. (2007) who reported that Spinosad was attractive to females of Aedes aegypti (Diptera: Culicidae). Another reason for the increased egg-laying by females might be due to Spinosad poisoning (Toews \& Subramanyam, 2003), which is disrupting the receptors sites of nerve cells of insects, exhibiting symptoms such as muscular contractions, protruding of the ovipositor and expelling of the eggs (Salgado, 1998). Similarly, the protrusion of 
the ovipositor of houseflies when exposed to Spinosad has been reported by Scott (1998). In another study, Yee (2018) reported that fruit fly, Rhagoletis indifferens Curran (Diptera: Tephritidae) were weakened after exposure to Spinosad and they were unable to oviposit or might lay eggs only at a low level. It should be noted that in our study, in some cases, no eggs were laid by females in the host plant soils treated with Spinosad. This behavior of fungus gnat females may be explained by Perez et al. (2007) who demonstrated the limited possibility of $A$. aegypti to spread eggs over various oviposition sites in one cage experiment or skip the egg-laying behavior by some $A$. aegypti.

In this study, although fungus gnat females were highly attracted to lay more eggs in the host plants parsley and poinsettia soils treated with Spinosad alone or in combination with the botanical compound, these eggs could not successfully finalize their life cycle to adults, resulting in a high reduction of adult emergence (> $72 \%$ ). This reduction might be due to the ability of Spinosad to maintain its insecticidal properties and persist for weeks, resulting in a suppression of fungus gnat adult emergence. These findings are again supported by Perez et al. (2007), who observed that the high reduction of $A$. aegypti larvae in Spinosad treatments (94 - $100 \%$ ). Moreover, Cloyd (2009) reported that Spinosad works quickly and is able to kill thrips Frankliniella occidentalis (Thripidae: Thysanoptera) within one or three days after either contact or ingestion. Use of this naturally derived insecticide for controlling thrips could be helpful to control other insect species in the greenhouse e.g. fungus gnats (Ludwig \& Oetting, 2001). Further investigations to determine the effectiveness of this insecticide to manage fungus gnats in the greenhouse are needed.

Additionally, the egg-laying behavior of females might be impacted by temperature. In this regard, our experiments with poinsettia host plants were conducted in November 2019 - January 2020. The oviposition of fungus gnat females might be negatively affected by temperature, thus females did not lay as many eggs in the host plants as compared to the trials performed with basil and parsley plants in May-June and August-September 2019, respectively. In another study, Pecora et al. (1989) suggested that the limited oviposition of Dasineura sp. near capsulae (Diptera: Cecidomyiidae) on poinsettia plants might be due to the deterrent compound or have a floral morphology, which might limit the attraction of females to the plants for oviposition. However, in parsley and poinsettia host plants, fungus gnat females laid more eggs in these host plants soils treated with Spinosad, while females were less stimulated to oviposit in the basil plant soils treated with Spinosad. Cloyd (2014) reported that some volatiles such as linalool, a monoterpene 
alcohol that is present in plants such as lavender, marjoram and basil can be used for repelling fungus gnat adults. However, further studies will be required to assess the efficacy of volatiles derived from host plants species tested on attraction and oviposition of fungus gnats.

\section{Metarhizium brunneum}

The egg-laying behavior of fungus gnat females was significantly reduced when exposed to the host plant soils treated with $M$. brunneum individually or in combination with the botanical capsules as compared to the control treatments. Similarly, the reduction of the ovipositional rate of Musca domestica females after exposure to $M$. anisopliae has been reported by GarciaMunguia et al. (2015). Baker et al. (2018) reported that the ovipositional rate of the same species was reduced when exposed to $M$. anisopliae. In another study, M. anisopliae has been shown to be effective in reducing fecundity $(58.4 \%-72.1 \%)$ and fertility $(28.6 \%-45.9 \%)$ of the fruit fly Ceratitis capitata (Diptera: Tephritidae) (Quesada-Moraga et al., 2006). Our results indicated that fungus gnat females preferred to lay more eggs in soils of the host plant species parsley and poinsettia treated with $M$. brunneum in combination with the botanical capsules as compared to the fungal suspension M. brunneum alone. However, in basil plants, M. brunneum alone was more attractive for fungus gnat females to oviposit as compared to the combined formulation of this fungal suspension with the botanical compounds. This egg-laying behavior of female fungus gnat in different host plant species might depend on many ecological, chemical and physiological factors (Braun et al., 2012).

All of the host plant species soils treated with M. brunneum individually or in combination with the botanical capsules resulted in a higher reduction of adult emergence in comparison to the control treatments. These results are in agreement with the findings of Tavoosi Ajvad et al. (2019) which found a high efficacy of the fungal strain M. anisopliae (> $50 \%$ ) as a biocontrol agent when applied alone or in combination with the predatory mite G. aculeifer against fungus gnat $L$. auripilla (Diptera: Sciaridae) in mushroom production. This reduction might be supported by Baker et al. (2018) which M. anisopliae infection might affect internally in dipteran species. In another study, it has been shown the M. anisopliae infection via the buccal cavity of Lucilia cuprina (Wiedemann) (Diptera: Calliphoridae) (Leemon \& Jonsson, 2012). Furthermore, M. anisopliae might cause muscle paralysis, resulting in muscles weakness in infected insects and death (Samuels et al. 1998). Short et al. (2010) pointed the efficacy of M. anisopliae in immune defense of dipteran species (e.g. M. domestica). Hence, the efficacy of entomopathogenic fungi (EPF) on 
various insects depends on many factors, which are related to the behavior of the insects in natural habitat (Gindin et al., 2006). In case of B. impatiens, where larvae hatched from eggs move through the substrate to find a suitable food source, they might come into contact with spores or Spinosad applied via the suspension into the substrate, resulting in an increased probability of larvae to be infected with the fungal strain or Spinosad. However, this idea needs further studies. Moreover, the reduction of adult emergence might be influenced by larval cannibalism in specific conditions (Wilkinson \& Daugherty, 1970).

Furthermore, the egg-laying behavior of fungus gnat females after exposure to potted poinsettia plants and soils treated with $M$. brunneum or Spinosad individually or in combination with the botanical capsules at different rates (experiment 4) did not provide a clear picture in terms of egg-laying behavior of fungus gnat females. We suspect that this obscure behavior of fungus gnat females in this trial might be as a result of the setup of this trial and placing the treated poinsettia plants closely in one cage experiment. Obviously, this experiment needs a repetition and a better design should be considered in the further tests.

The egg-laying behavior of female fungus gnat in this study varied based on the different factors such as volatiles emitted from host plants, growing medium, volatiles generated from microbial products, environmental condition and a combination of factors. In this regard, Braun et al. (2012) reported a strong ovipositional preference of fungus gnat females to another species of EPF e.g. B. bassiana. According to the study of Binns (1973), the presence of host plant species might have influenced the effect of the bioinsecticides tested on fungus gnats. For instance, Jagdale et al. (2004) reported a higher efficacy of an entomopathogenic nematode (EPN) (Steinernema feltiae) in reducing larval fungus gnat B. coprophila in New Guinea impatiens (Impatiens hawkeri) (55\%) as compared to the poinsettia (E. pulcherrima) (18\%). Moreover, there have been some previous reports on the efficacy of biocontrol agents alone or in combined formulations to suppress fungus gnat populations in nursery and greenhouse crops. Bennison et al. (2008) demonstrated that an efficient reduction of fungus gnats has been found in parsley ( $P$. crispum) plants after using rove beetles Dalotia coriaria adults as a biological control. VillanuevaSánchez et al. (2017) reported the susceptibility of B. difformis larvae to entomopathogenic nematodes (EPNs) Heterorhabditis bacteriophora and S. feltiae in poinsettia plants. In another study, the combination of EPN S. feltiae and thiamethoxam has been shown to be highly effective (> $90 \%$ ) against larvae of the fungus gnat species B. odoriphaga in Chinese chive (Allium tuberosum Rottl. ex Spreng.) (Wu et al., 2017). 
The findings of this study indicate the potential of biopesticides, specifically Spinosad in combination with the botanical capsule Minx when applied in host plant soils to suppress fungus gnats. These combined formulations suggested an "attract and kill" strategy in the host plant species e.g. basil, parsley and poinsettia, which effectively reduce fungus gnat populations.

\section{Conclusion}

This is the first study that demonstrated that the efficacy of biopesticides $M$. brunneum and Spinosad individually or in combination with the botanical capsules Minx on attraction and oviposition of the fungus gnat B. impatiens in three host plants (basil, parsley and poinsettia) in the greenhouse conditions. This study showed that fungus gnat females laid more eggs in parsley and poinsettia plants soils treated with Spinosad alone or in combination with the botanical capsules as compared to the other treatments. However, these eggs could not successfully finalize their cycle to adults, resulting in a higher reduction of adult emergence. This result points to an additive effect of this co-formulation as shown by the reduced number of adults emerging in an "attract and kill" strategy. From a practical standpoint, further investigations are required to develop these co-formulations (e.g. biopesticides M. brunneum or Spinosad in combination with the botanical capsules Minx) in suppressing fungus gnat populations in greenhouse production systems.

\section{Acknowledgements}

We would like to thank Bianca Tappe for their technical assistance. We would like to thank Jörg Rademacher (Katz Biotech AG, Baruth, Germany) for providing the botanical capsules. Furthermore, we would like to thank Maik Knobel for providing the poinsettia plants.

\section{References}

Acharya, R., Hwang, H. S., Shim, J. K., Yu, Y. S. \& Lee, K. Y. (2019) Control efficacy of fungus gnat, Bradysia impatiens, enhanced by a combination of entomopathogenic nematodes and predatory mites. Biological Control, 138.

Andreadis, S. S., Cloonan, K. R., Bellicanta, G. S., Paley, K., Pecchia, J. \& Jenkins, N. E. (2016) Efficacy of Beauveria bassiana formulations against the fungus gnat Lycoriella Ingenua. Biological Control, 103, 165-71. 
Baker, D. K., Rice, S. J., Leemon, D. M. \& James, P. J. (2018) Horizontal transmission of Metarhizium anisopliae (Hypocreales: Clavicipitacea) and the effects of infection on oviposition rate in laboratory populations of Musca domestica (Diptera: Muscidae). Pest Management Science, 74, 987-991.

Bartlett, G. R. \& Keil, C. B. O. (1997) Identification and characterization of a permethrin resistance mechanism in populations of the fungus gnat Lycoriella mali (Fitch) (Diptera: Sciaridae). Pesticide Biochemistry and Physiology, 58, 173-181.

Bethke, J. A. \& Dreistadt, S. H. (2013) Pest notes: Fungus gnats. University of California Statewide integrated pest management program, Davis, CA.

Bennison, J., Maulden, K., Maher, H. \& Tomiczek, M. (2008) Development of a grower rearingrelease system for Atheta coriaria, for low cost biological control of ground dwelling pest life stages. IOBC/wprs Bulletin, 32.

Binns, E.S. (1973) Fungus gnats (Diptera: Mycetophilidae, Sciaridae) and the role of mycophagy in soil: a review. Revue d'Ecologie et de Biologie du Sol, 18, 77-90.

Braun, S. E., Sanderson, J. P. \& Wraight, S. P. (2012) Larval Bradysia impatiens (Diptera: Sciaridae) potential for vectoring Pythium root rot pathogens. Phytopathology, 102, 283-289.

Braun, S. E., Sanderson, J. P., Daughtrey, M. L. \& Wraight, S. P. (2012) Attraction and oviposition responses of the fungus gnat Bradysia impatiens to microbes and microbe-inoculated seedlings in laboratory bioassays. Entomologia Experimentalis et Applicata, 145, 89-101.

Bret, B. L., Larson, L. L., Schoonover, J. R., Sparks, T. C. \& Thompson, G. D. (1997) Biological properties of Spinosad. Down to Earth, 52, 6-13.

Chambers, R. J., Wright, E. M. \& Lind, R. J. (1993) Biological control of glasshouse sciarid flies (Bradysia spp.) with the predatory mite, Hypoaspis miles, on cyclamen and poinsettia. Biocontrol Science and Technology, 3, 285-293.

Cloyd, R. A. (2000) Fungus gnat and shore fly management strategies: panel discussion. In: Proceedings for the $16^{\text {th }}$ conference on insect and disease management on ornamentals. San Jose, CA. Society of American Florists, Alexandria, VA.

Cloyd, R. A. \& Zaborski, E. R. (2004) Fungus gnats, Bradysia spp. (Diptera: Sciaridae), and other arthropods in commercial bagged soilless growing media and rooted plant plugs. Journal of Economic Entomology, 97, 503-510. 
Cloyd, R. A. \& Dickinson, A. (2006) Effect of Bacillus thuringiensis subsp. israelensis and neonicotinoid insecticides on the fungus gnat Bradysia sp. nr. Coprophila (Lintner) (Diptera: Sciaridae). Pest Management Science, 62, 171-177.

Cloyd, R. A. (2008) Management of fungus gnats (Bradysia Spp.) in greenhouses and nurseries. Floriculture and Ornamental Biotechnology, 2, 84-89.

Cloyd, R. A. (2009) Western flower thrips (Frankliniella occidentalis) management on ornamental crops grown in greenhouses: Have we reached an impasse. Pest Technology, 3, 1-9.

Cloyd, R. A. (2014) Horticultural Entomology/Plant Protection, Department of Entomology, Kansas State University, Manhattan, KS, USA. Personal observation.

Cox, D. \& Craker, L. (1994b) Herbs: Grow them and they will sell. Greenhouse Manager, 12, 74-77.

Darriet, F., Duchon, S. \& Hougard, J. M. (2005) Spinosad: a new larvicide against insecticideresistant mosquito larvae. Journal of the American Mosquito Control Association, 21, 495496.

Eger, J. R. J. E. \& Lindenberg, L. B. (1998) Utility of Spinosad for insect control in Florida vegetables. Proc. Florida State Horticulture, 111, 55-57.

El-Hamalawi, Z. A. (2008) Acquisition, retention and dispersal of soilborne plant pathogenic fungi by fungus gnats and moth flies. Annals of Applied Biology, 153, 195-203.

Elmer, W. H. (2008) Preventing spread of Fusarium wilt of Hiemalis begonias in the greenhouse. Crop Protection, 27, 1078-1083.

El-Sayed, A. M., Suckling, D. M., Byers, J. A., Jang, E. B. \& Wearing, C. H. (2009) Potential of 'lure and kill' in long-term pest management and eradication of invasive species. Journal of Economic Entomology, 102, 815-835.

Erler, F., Polat, E., Demir, H., Cetin, H. \& Erdemir, T. (2009) Evaluation of microbial products for the control of the mushroom phorid fly, Megaselia Halterata (Wood). Journal of Entomological Science, 44, 89-97.

Freire, R. A., de Moraes, G. J. , Silva, E. S., Vaz A. C. \& de Campos Castilho, R. (2007) Biological control of Bradysia matogrossensis (Diptera: Sciaridae) in mushroom cultivation with predatory mites. Experimental and Applied Acarology, 42, 87-93.

Garcia-Munguia, C. A., Reyes-Villanueva, F., Rodriguez-Perez, M. A., Cortez-Madrigal, H., AcostaRamos, M., Ibarra-Juarez, L. A., Velázquez-Machuca, M. A., Silva-García, J. T., Rebollar-Plata, M. \& Garcia-Munguia, A. M. (2015) Autodissemination of Metarhizium anisopliae and 
Beauvaria bassiana in Musca domestica L. results in less oviposition and short gonotrophic cycle. Southwestern Entomologist, 40, 519-529.

Gauthier, N. L. (1989) a new alternative to chemicals for fungus gnat control. Connecticut Greenhouse Newsletter, 151, 13-16.

Gindin, G., Levski, S., Glazer, I. \& Soroker, V. (2006) Evaluation of the entomopathogenic fungi Metarhizium anisopliae and Beauveria bassiana against the red palm weevil Rhynchophorus ferrugineus. Phytoparasitica, 34, 370-379.

Golo, P. S., Gardner, D. R., Grilley, M. M., Takemoto, J. Y., Krasnoff, S. B., Pires, M. S., Fernandes, E. K. K., Bittencourt, V. R. E. P. \& Roberts, D. W. (2014) Production of destruxins from Metarhizium spp. fungi in artificial medium and in endophytically colonized cowpea plants. PLoS One, 9, e104946.

Gouge, D. H. \& Hague, N. G. M. (1995) Glasshouse control of fungus gnats, Bradysia pauper, on fuchsias by Steinernema feltiae. Fundamentals in Applied Nematology, 18, 77-80.

Hamlen, R. A. \& Mead, F. W. (1979) Fungus gnat larval control in greenhouse plant production. Journal of Economic Entomology, 72, 269-271.

Harris, M. A., Oetting, R. D. \& Gardner, W. A. (1995) Use of entomopathogenic nematodes and a new monitoring technique for control of fungus gnats, Bradysia Coprophila (Diptera: Sciaridae), in floriculture. Biological Control, 5, 412-418.

Jagdale, G. B., Casey, M. L., Grewal, P. S. \& Lindquist, R. K. (2004) Application rate and timing, potting medium, and host plant effects on the efficacy of Steinernema feltiae against the fungus gnat, Bradysia coprophila, in floriculture. Biological Control, 29, 296-305.

Jagdale, G. B., Casey, M. L., Cañas, L. \& Grewal, P. S. (2007) Effect of entomopathogenic nematode species, split application and potting medium on the control of the fungus gnat, Bradysia difformis (Diptera: Sciaridae), in the greenhouse at alternating cold and warm temperatures. Biological Control, 43, 23-30.

Jess, S. \& Bingham, J. F. W. (2004) Biological control of sciarid and phorid pests of mushroom with predatory mites from the genus Hypoaspis (Acari: Hypoaspidae) and the entomopathogenic nematode Steinernema feltiae. Bulletin of Entomological Research, 94, 159-167.

Katumanyane, A., Ferreira, T. \& Malan, A. P. (2018) Potential use of local entomopathogenic nematodes to control Bradysia impatiens (Diptera: Sciaridae) under laboratory conditions. African Entomology, 26, 337-349. 
Kennedy, M. K. (1976) The interaction of Bradysia impatiens (Joh.) (Diptera: Sciaridae), a fungal host, and the root systems of vascular plants. PhD Dissertation, Cornell University, Ithaca, NY, USA.

Kim, H. H., Choo, H. Y., Lee, H. S., Park, C. G., Lee, D. W., Jin, B. R. \& Choo, Y. M. (2001) Biological control of Lycoriella mali (Diptera: Sciaridae), a pest of oyster mushroom, Pleurotus ostreatus using entomopathogenic nematodes. Korean Journal of Applied Entomology, 40, 59-67.

Leemon, D. M. \& Jonsson, N. N. (2012) Comparative studies on the invasion of cattle ticks (Rhipicephalus (Boophilus) microplus) and sheep blowflies (Lucilia cuprina) by Metarhizium anisopliae (Sorokin). Journal of Invertebrate Pathology, 109, 248-259.

Ludwig, S. W. \& Oetting, R. D. (2001) Evaluation of medium treatments for management of Frankliniella occidentalis (Thripidae: Thysanoptera) and Bradysia coprophila (Diptera: Sciaridae). Pest Management Science, 57, 1114-1118.

Ma, J., Chen, S. L., Moens, M., Han, R. \& De Clercq, P. (2013a) Efficacy of entomopathogenic nematodes (Rhabditida: Steinernematidae and Heterorhabditidae) against the chive gnat, Bradysia odoriphaga. Journal of Pest Science, 86, 551-561.

Menzel, F., Smith, J. E. \& Colauto, N. B. (2003) Bradysia difformis Frey and Bradysia ocellaris (Comstock): two additional neotropical species of black fungus gnats (Diptera: Sciaridae) of economic importance: a redescription and review. Annals of Entomological Society of America, 94, 448-457.

Mertz, F. P. \& Yao, R. C. (1990) Saccharopolyspora spinosa sp. nov. isolated from soil collected in a sugar rum still. International Journal of Systematic Bacteriology, 40, 34-39.

Mohrig, W. \& Menzel, F. (2010) Sciaridae (black fungus fungus gnats). In: Brown, B.V., Borkent, A., Cumming, J. M., Wood, D. M., Woodley, N. E. \& Zumbado, M. A. (eds.) Manual of Central American Diptera. NRC Research Press, Ottawa, Canada, 279-292.

Mohrig, W., Heller, K., Hippa, H. Vilkamaa, P. \& Menzel, F. (2013) Revision of the black fungus gnats (Diptera: Sciaridae) of North America. Studia dipterologica, 19, 141-286.

Osborne, L. S., Boucias, D. G. \& Lindquist, R. K. (1985) Activity of Bacillus thuringiensis var. israelensis on Bradysia coprophila (Diptera: Sciaridae). Journal of Economic Entomology, 78, 922-925.

Pecora, P., Cristofaro, M. \& Stazi, M. (1989) Dasineura sp. near capsulae (Diptera: Cecidomyiidae), a candidate for biological control of Euphorbia esula complex in North America. Annals of the Entomological Society of America, 82, 693-700. 
Perez, C. M., Marina, C. F., Bond, J. G., Rojas, J. C., Valle, J. \& Williams, T. (2007) Spinosad, a naturally derived insecticide, for control of Aedes aegypti (Diptera: Culicidae): efficacy, persistence, and elicited oviposition response. Journal of Medical Entomology, 44, 631-638.

Popko, D. A., Henke, J. A., Mullens, B. A. \& Walton, W. E. (2018) Evaluation of two entomopathogenic fungi for control of Culex quinquefasciatus (Diptera: Culicidae) in underground storm drains in the Coachella Valley, California, United States. Journal of Medical Entomology, 55, 654-665.

Quesada-Moraga, E., Ruiz-García, A. \& Santiago-Alvarez, C. (2006) Laboratory evaluation of entomopathogenic fungi Beauveria bassiana and Metarhizium anisopliae against puparia and adults of Ceratitis capitata (Diptera: Tephritidae). Journal of Economic Entomology, 99, 19551966.

Romi, R., Proietti, S., Di Luca, M. \& Cristofaro, M. (2006) Laboratory evaluation of the bioinsecticide Spinosad for mosquito control. Journal of the American Mosquito Control Association, 22, 93-96.

Salgado, V. L. (1998) Studies on the mode of action of Spinosad: insect symptoms and physiological correlates. Pesticide Biochemistry and Physiology, 60, 91-102.

Samuels, R. I., Reynolds, S. E. \& Charnley, A. K. (1998) Calcium channel activation of insect muscle by destruxins, insecticidal compounds produced by entomopathogenic fungus Metarhizium anisopliae . Comparative Biochemistry and Physiology - Part C: Toxicology and Pharmacology, $90,403-412$.

San-Blas, E., Luzardo, M., Portillo, E., Fuenmayor, Y. \& Bastidas, B. (2017) Heterorhabditis amazonensis for biological control of fungus gnats Bradysia difformis in Daisy gerberas. BioControl, 62, 847-855.

Scott, J. G. (1998) Toxicity of Spinosad to susceptible and resistant strains of house flies, Musca domestica. Pesticide Science, 54, 131-133.

Short, S. M. \& Lazzaro, B. P. (2010) Female and male genetic contributions to post-mating immunedefence in female Drosophila melanogaster, Proceedings of the Royal Society BBiological Sciences, 277, 3649-3657.

Tavoosi Ajvad, F., Madadi, H., Michaud, J. P., Zafari, D. \& Khanjani, M. (2019) Combined applications of an entomopathogenic fungus and a predatory Mite to control fungus gnats (Diptera: Sciaridae) in mushroom production. Biological Control, 141. 
Thompson, G. D., Dutton, R. \& Sparks, T. C. (2000) Spinosad - a case study: an example from a natural products discovery programme. Pest Management Science, 56, 696-702.

Toews, M. D. \& Subramanyam, B. (2003) Contribution of contact toxicity and wheat condition to mortality of stored-product insects exposed to Spinosad. Pest Management Science, 59, 538544.

Villanueva-Sánchez, E., Guzmán-Franco, A. W, Lomelí-Flores, J. Refugio, Alatorre-Rosas, R., OrtízSolorio, C. A, Manuel-Pinto, V. \& Villanueva-Verduzco, C. (2017). Susceptibility of Bradysia difformis to entomopathogenic nematodes and their persistence in substrates used for poinsettia production. Southwestern Entomologist, 42, 1015-1026.

Wu, H., Gong, Q., Fan, K., Sun, R., Xu, Y. \& Zhang, K. (2017) Synergistic effect of entomopathogenic nematodes and thiamethoxam in controlling Bradysia odoriphaga Yang and Zhang (Diptera: Sciaridae). Biological Control, 111, 53-60.

Wilkinson, J. D. \& Daugherty, D. M. (1970a) The biology and immature stages of Bradysia impatiens (Diptera: Sciaridae). Annals of the Entomological Society of America, 63, 656-660.

Yan, X., Zhao, G. Y. \& Han, R. C. (2019). Integrated management of chive gnats (Bradysia odoriphaga Yang \& Zhang) in chives using entomopathogenic nematodes and low-toxicity insecticides. Insects, 10, 161.

Yee, W. L. (2018) Spinosad versus spinetoram effects on kill and oviposition of Rhagoletis indifferens (Diptera: Tephritidae) at differing fly ages and temperatures. Journal of Insect Science, 18.

Yousef, M., Lozano-Tovar, M. D., Garrido-Jurado, I., \& Quesada-Moraga, E. (2013). Biocontrol of Bactrocera oleae (Diptera: Tephritidae) with Metarhizium brunneum and its extracts. Journal of Economic Entomology, 106, 1118-1125. 


\section{General discussion}

The dark-winged fungus gnats (Diptera: Sciaridae) are diverse and specious. Fungus gnats such as the species, Bradysia impatiens Johannsen are considered as major insect pests in greenhouses and nurseries. Various approaches for the control of fungus gnats are available, but novel and environmentally safe control strategies are desirable for efficient management of fungus gnats in greenhouses. Biological control agents (BCAs) including Bacillus thuringiensis Bt (Harris et al., 1995), entomopathogenic nematodes (EPNs) (Jagdale et al., 2004) and predatory mites (Jess \& Bingham, 2004) could provide a potential alternative to chemical pesticides. Finding new biological formulations principally derived from fungi or bacteria could be helpful to supply alternative control strategies implemented in the integrated pest management (IPM) to control fungus gnat $B$. impatiens.

Survival larvae on pure fungal cultures or in the substrate treated with the fungal cultures (Chapter 1)

This study showed that fungus gnat larvae are able to survive after feeding on pure fungal cultures (Metarhizium brunneum, Beauveria bassiana and Acremonium strictum). Fungus gnat larvae were more susceptible to $M$. brunneum as compared to other fungal strains, which resulted in a low survival rate of fungus gnat larvae. However, some fungal isolates of Beauveria bassiana and Acremonium strictum allowed a high percentage of larval survival. In this regard, Andreadis et al. (2016) indicated that eggs and larvae of Lycoriella ingenua Dufour (Diptera: Sciaridae) were not susceptible to infection by $B$. bassiana strain GHA; hence, fungus gnat larvae exhibited a high survivorship. Additionally, the efficacy of the fungal suspensions of $M$. brunneum ( $\mathrm{Cb} 15), B$. bassiana (EABb04/01-Tip) and $A$. strictum, when mixed within the substrate against fungus gnat larvae were evaluated. Fewer numbers of adults emerged from larvae exposed to the substrate treated with M. brunneum and A. strictum (22\% to $25 \%$ ) in comparison to B. bassiana. Tavoosi Ajvad et al. (2019) reported $>50$ \% larval mortality of fungus gnat $L$. auripilla after exposure to $M$. anisopliae $\left(10^{7}\right.$ and $10^{8}$ spores $\left./ \mathrm{ml}\right)$ applied to the compost of mushroom production. However, the study of Dobrindt (2013) indicated that M. anisopliae at a rate of $1.6 \times 10^{8}$ spores $/ \mathrm{ml}$ applied to the growing medium did not significantly reduce the emergence of fungus gnat adults Bradysia spp. The efficacy of Metarhizium might be influenced by the strains of the fungus, the application method and the time between fungus and larvae application and soil substrate type. 
A number of factors such as transferring larvae from stock-rearing medium, lack of favorite microbial food source and some intrinsic unidentified biological factors could influence the development of fungus gnat larvae (Olson et al., 2002). According to Wilkinson \& Daugherty, (1970a), fourth instars of larvae were able to cannibalize the weakened larvae and pupae, which might result in a reduced number of adults emerging. The growing medium type and its components, the availability of plant material or fungal growth as a food source in the growing medium are also critical with regard to larval growth and development (Olson, et al., 2002, Meers \& Cloyd, 2005, Cloyd \& Dickinson, 2008). Therefore, the fungus gnat populations could be suppressed by a number of factors. Regarding the growing medium type, Fruhstorfer Erde Typ $\mathrm{P}$ was evaluated in our study as the more suitable substrate for development of fungus gnat larvae. The high adult emergence from the substrate Fruhstorfer Erde Typ P might be a result of microbial activity and fine texture of this substrate.

Furthermore, the addition of the fertilizer in the substrate did not affect the abundance of fungus gnats. In this case, the development of fungus gnat larvae might be mainly correlated with the presence of the decaying plant material and microbial food sources (e.g. fungi or bacteria) in the soil rather than the fertilizer. However, this side of study needs further testing.

Efficacy of biopesticides (Bacillus thuringiensis Bt and M. brunneum) in combination with the botanical compounds against fungus gnat larvae (Chapter 2)

Fungus gnat larvae exposed to the botanical microcapsule Minx showed a positive interaction with the Minx capsules encapsulated with Na-alginate. According to our laboratory experiments using water agar, fungus gnat larvae move straightly towards the Minx capsules and remain a certain times around of this botanical capsule to find a food source, but larva moved away later from the Minx capsules. The semiochemical emitted from the alginate beads might be useful to attract insects in a biological control approach (Heuskin et al., 2012). Beside larvae, fungus gnat adults showed a strong ovipositional preference for Minx capsules using multiple-choice experiments.

A few numbers of larvae after exposure to the biopesticides Bacillus thuringiensis Bt or $M$. brunneum CB15 could finalize their life cycle to adults, resulting in a reduction of adult emergence. A combined application of Bt or M. brunneum with the Minx capsules indicated an additive effect to control fungus gnat larvae, which resulted in a higher reduction of adult emergence (37\% and $30 \%$, respectively) as compared to $\mathrm{Bt}$ alone (42\%) or single fungal treatment (33\%). This reduction might be due to the hyperactivity and the emigration of $B$. impatiens larvae towards the 
botanical capsules (Minx) (= an attractive agent) through the substrate; thus increasing the probability of larvae to be infected by a fungal spore or Bt suspensions applied in the substrate via contact or ingestion. Specifically M. brunneum in combination with the Minx capsules was slightly more effective (70 \%) in suppressing the adult emergence. The low efficacy of Bt treatments in fungus gnats control could be related to a short residual activity of Bt in the substrate and its adsorption to the substrate particles, which causes a rapid reduction in toxicity (Margalit et al., 1985). However, there is a wide variation in Bt efficacy for fungus gnat control. Osborne et al. (1985) demonstrated the high influence of Bt against larval fungus gnats $B$. coprophila, while some studies reported minimal or no efficacy of Bt against fungus gnat larvae (Cloyd \& Dickinson, 2006, Koller, 2011). This wide variation in Bt efficacy might be affected by a number of factors including type of formulation (type of Bt product), dose application, the larval instar and type of substrate (Molloy et al., 1981).

The implementation of a combined application of a biopesticide and the botanical compound Minx resulted in a better effect to control fungus gnats. Fewer numbers of adults did emerge from these combined formulations as compared to single fungal or Bt treatments. Hence, these results point to an "attract and kill" strategy, which might efficiently reduce fungus gnat populations. Thus, these combined formulations might be used as an alternate pest control approach to combat fungus gnats populations in the greenhouses.

Efficacy of biopesticides (M. brunneum and Spinosad) in combination with the botanical capsules Minx on larval fungus gnats and oviposition of fungus gnat females (Chapter 3 )

Chapter 3 extended the implementation of an "attract and kill" approach through a combination of biopesticides (e.g. M. brunneum and Spinosad) and the botanical capsules Minx to control fungus gnats.

The combined applications of a biopesticide (e.g. M. brunneum, Bt and Spinosad) and the botanical capsule Minx were found to be attractive for female fungus gnats to oviposit. Fungus gnat females showed a strong ovipositional preference for Spinosad treatments individually or in combination with the Minx capsules in comparison to the other biopesticides. The increased egglaying behavior of female fungus gnats in Spinosad treatments might be due to Spinosad poisoning. This hypothesis is supported by the study of Toews \& Subramanyam (2003) who found that Spinosad influences the receptor sites of nerve cells of Rhyzopertha dominica, resulting in poisoning symptoms such as muscle paralysis, protruding of the ovipositor and expelling of the 
eggs. In similar work, these poisoning symptoms were found in houseflies when exposed to Spinosad (Scott, 1998). Interestingly, these eggs laid by females in Spinosad treatments could not finalize their life cycle to adults, causing an efficient reduction of adult emergence as compared to the Bt or fungal treatments. This high efficacy of Spinosad in the reduction of adult emergence is presumably due to the presence of Spinosad residues. According to the findings of Perez et al. (2007), Spinosad is able to retain its insecticidal properties for weeks, which is sufficient to suppress the development of $A$. aegypti larvae. The results of our study demonstrated that Spinosad in combination with the botanical capsules Minx might be used as a "trap crop strategy", highly attractive for oviposition of fungus gnats; thus being kept away from the main crops in greenhouses and lured to trap pots containing Spinosad and the botanical capsules.

Additionally, volatiles emitted from the botanical capsules (Minx) can be seen as a stimulant attractant for female fungus gnats to oviposit. The egg-laying behavior of fungus gnat females depend on the volatiles emitted from the components of the growing medium or microbial activity that determines where fungus gnats lay eggs (Olson et al., 2002, Meers \& Cloyd, 2005). In the present study, the different rates of the botanical capsules Minx showed a positive effect on the fungus gnat larvae, with no significant differences between the Minx capsules in terms of adult emergence. Therefore, the botanical capsules containing $0.1 \%$ mint oil (minimum rate) have been found to be an excellent attractant to fungus gnat larvae.

In case of the application of EPF M. brunneum in this study, the comparison of two concentrations, $1 \times 10^{7} \mathrm{spore} / \mathrm{ml}$ and $1 \times 10^{9} \mathrm{spore} / \mathrm{ml}$ of $M$. brunneum against fungus gnat larvae concluded that both concentrations decrease the adult emergence of fungus gnat as compared to the control treatments. The minimum concentration of $M$. brunneum $\left(1 \times 10^{7} \mathrm{spore} / \mathrm{ml}\right)$ was considered for this study due to the technical aspects to prepare this fungal suspension and the sufficient efficacy (Vega et al., 2008). Similarly, larval fungus gnat L. auripilla showed high susceptibility (> $50 \%$ mortality) to infection by $M$. anisopliae at rates of $10^{7}$ and $10^{8} \mathrm{spores} / \mathrm{ml}$ when applied to the compost of mushroom production (Tavoosi Ajvad et al., 2019).

In our study on Spinosad application against fungus gnat larvae, we found that Spinosad significantly suppressed the adult emergence of $B$. impatiens. This reduction might be related to the residual activity of Spinosad (up to two weeks) in the substrate. The ability of Spinosad to persist in the substrate for weeks might be effective in controlling the development of fungus gnat larvae. This finding was supported by Perez et al. (2007) who reported an efficient reduction of $A$. aegypti (L.) (Diptera: Culicidae) larvae (94-100\%) in the presence of residues from the Spinosad 
treatments. Additionally, Spinosad has been shown to be effective by stimulating the insect nervous system and causing paralysis and death (Salgado, 1998, Orr et al., 2009).

The combined application of biopeticides ( $M$. brunneum or Spinosad) and the botanical compound Minx slightly enhanced the control efficacy against fungus gnats as shown by the reduced number of emerged adults. In this regard, some studies have recently showed the higher efficacy of the combined application of different, but compatible products for suppressing fungus gnat populations. Acharya et al. (2019) obtained the enhanced control efficacy in fungus gnat $B$. impatiens by combining an EPN (Heterorhabditis indica) and the predatory mite (Stratiolaelaps scimitus). In other study, the combination of the fungal strain $M$. anisopliae with the predatory mite Gaeolaelaps aculeifer could be used to improve the control efficacy of fungus gnat L. auripilla (Diptera: Sciaridae) (Tavoosi Ajvad et al., 2019).

Combined formulations tested in this study, specifically Spinosad either when applied alone or in combination with the botanical microcapsule Minx significantly reduced the number of fungus gnat adults as compared to the fungal treatments. The high efficacy of the combined formulation might be related to the movement of $B$. impatiens larvae towards the Minx capsules through the substrate, where it is possible that larvae come into contact with spores or Spinosad applied via the suspension into the substrate, resulting in an infection by the fungal spores or Spinosad. In this regard, volatiles elicited from the botanical Minx capsules and biopesticide Spinosad containing a specific aroma derived from Actinomycetes (Perez et al., 2007) might play a pivotal role in attraction of fungus gnat larvae. The application of biopesticides, specifically Spinosad, in combination with the botanical capsules Minx pointed towards an "attract and kill" strategy, which might efficiently reduce fungus gnat populations.

Efficacy of biopesticides (M. brunneum and Spinosad) in combination with the Minx capsules to control fungus gnat on different host plants (Chapter 4)

The ovipositional preference of fungus gnat females varied according to the biopesticides when applied alone or in combination with the botanical compound. This variation might be due to the volatiles generated from the host plant species or a result of microbial activity in the growing medium, substrate type and the components (Evans et al., 1998, Olson et al., 2002). Using basil plants, females showed a strong ovipositional preference in the substrate treated with the botanical capsule Minx or the control treatment. However, linalool as a monoterpene alcohol, which can be found naturally in basil plants, has been reported to repel fungus gnat adults 
(Narusuye et al., 2005, Cloyd et al., 2010). The underlying mechanism for interaction between volatiles elicited from host plant species tested in this study and fungus gnat $B$. impatiens was not studied. Therefore, this side of our study requires further investigations.

In addition, Fungus gnat females are attracted to oviposit in parsley and poinsettia plant substrates treated with Spinosad alone or in combination with the Minx capsules as compared to the fungal or control treatments. These findings are supported by Perez et al. (2007), who reported that Spinosad is attractive for females of $A$. aegypti (L.) to oviposit because of a distinctive aroma of damp earth derived from the Actinomycetes. However, the mechanism of selection an ovipositional site by female fungus gnats is a complex process, with a number of different biological, physical and chemical factors involved (Braun et al., 2012).

Eggs laid by fungus gnat females in the host plants species treated with Spinosad alone or in combination with the minx capsules did not finalize their life cycle to adults. Among host plant species, poinsettia plant soils treated with Spinosad individually (92\%) or in combination with botanical Minx capsules (80 \%) resulted in a higher reduction of adult emergence in comparison to the other host plants. This reduction might be due to the ability of Spinosad to persist in the substrates, which could be helpful to suppress the development of fungus gnat. However, closer examination of these results indicated that in two host plant species, parsley and poinsettia, Spinosad individually could cause a higher reduction of adults emergence, although not significantly, as compared to Spinosad in combination with the Minx capsule. This might be due to the physical and chemical traits of the host plant species, which might have an impact on the efficiency of the control agent (e.g. Spinosad) and as a consequence, the development and reproduction of fungus gnats.

Furthermore, female fungus gnats when exposed to different rates of Minx capsules containing a high rate of mint oil (10\%) did not show obviously their ovipositional preference on different treatments. It might be related to the placing the treated poinsettia plants closely in one experimental chamber. Hence, it requires repeating this experiment with a new setup.

\section{Conclusion}

Fungus gnat larvae of the species Bradysia impatiens, are able to survive on pure fungal diets Metarhizium brunneum, Beauveria bassiana and Acremonium strictum. Fungus gnat larvae were more susceptible to $M$. brunneum as compared to other fungal strains, resulting in fewer numbers of adults emerging. This susceptibility of larvae was also observed when the fungal suspension of 
M. brunneum was applied to the growing medium. In addition, larvae exposed to the Minx capsules containing mint oil showed a positive interaction with the Minx capsules. Furthermore, our hypothesis regarding the increased control efficiency of fungus gnat could be supported by a combination of an attractant (= Minx capsule) with a killing agent (= M. brunneum or Spinosad) in an "attract and kill" strategy. This combined formulation could provide a framework for further studies on the application of a biopesticide with Minx capsules to deal with populations of fungus gnats. Specifically, the higher efficacy of Spinosad in combination with Minx capsules might be due to this finding that Spinosad could maintain its insecticide properties for weeks, resulting in suppressing the development of larvae. However, Spinosad was found strongly attractive for fungus gnat females to oviposit, while these eggs did not fully develop to adults. Therefore, the combination of Spinosad with the Minx capsules suggests a "trap crop strategy". In addition, the combined application of biopesticides ( $M$. brunneum or Spinosad) with Minx capsules could efficiently reduce the emergence of fungus gnat adults on different host plant species (basil, parsley and poinsettia). Further studies need to improve the control efficiency of fungus gnats by combining the biopesticides with the botanical compound targeting the practical control strategy for fungus gnat $B$. impatiens control in greenhouse production systems. Furthermore, different combined formulations, for example entomopathogenic nematodes (EPNs) with Minx capsules, might pave the way for a new approach to manage fungus gnats. 


\section{References: General introduction and general discussion}

Acharya, R., Hwang, H. S., Shim, J. K., Yu, Y. S. \& Lee, K. Y. (2019) Control efficacy of fungus gnat, Bradysia impatiens, enhanced by a combination of entomopathogenic nematodes and predatory mites. Biological Control, 138.

Anas, O. \& Reeleder, R. D. (1988) Feeding habits of Bradysia coprophila on fungi and plant tissue. Phytoprotection, 69, 73-78.

Andreadis, S. S., Cloonan, K. R., Bellicanta, G. S., Paley, K., Pecchia, J. \& Jenkins, N. E. (2016) Efficacy of Beauveria bassiana formulations against the fungus gnat Lycoriella ingenua. Biological Control, 103, 165-171.

Bartlett, G. R. \& Keil, C. B. (1997) Identification and characterization of a permethrin resistance mechanism in populations of the fungus gnat Lycoriella mali (Fitch) (Diptera: Sciaridae). Pesticide Biochemistry and Physiology, 58, 173-181.

Braun, S. E. (2011) Elucidation of the role played by fungus gnats (Diptera: Sciaridae) in the transmission of Pythium root rot diseases of floriculture crops. PhD dissertation, Cornell University.

Braun, S. E., Sanderson, J. P., Daughtrey, M. L. \& Wraight, S. P. (2012) Attraction and oviposition responses of the fungus gnat Bradysia impatiens to microbes and microbe-inoculated seedlings in laboratory bioassays. Entomologia Experimentalis et Applicata, 145, 89-101.

Brues, C, T. (1946) Insect Dietary. An account of the food habits of insects, Harvard University press, Cambridge, MA, 466 PP.

Butt, T. M., Jackson, C. \& Magan, N. (2001) Fungi as biocontrol agents: progress, problems and potential. CABI Publishing, Wallingford, UK, $390 \mathrm{p}$

Carney, V. A., Diamond, J. C., Muphy, G. D. \& Marshall, D. (2002). The potential of Atheta coriaria Kraatz (Colepotera: staphylinidae) as a biological control agent for use in greenhouse crops. IOBC/WPRS Bulletin, 25, 37-40.

Chambers, R. J., Wright, E. M. \& Lind, R. J. (1993) Biological control of glasshouse sciarid flies (Bradysia spp.) with the predatory mite, Hypoaspis miles, on cyclamen and poinsettia. Biocontrol Science and Technology, 3, 285-293.

Cloyd, R. A. (2000) Fungus gnat and shore fly management strategies: panel discussion. In: Proceedings for the $16^{\text {th }}$ conference on insect and disease management on ornamentals. San Jose, CA. Society of American Florists, Alexandria, VA. 
Cloyd, R. A. \& Dickinson, A. (2006) Effect of Bacillus thuringiensis subsp. israelensis and neonicotinoid insecticides on the fungus gnat Bradysia sp nr. coprophila (Lintner) (Diptera: Sciaridae). Pest Management Science, 62, 171-177.

Cloyd, R. A., Dickinson, A., \& Kemp, K. E. (2007) Effect of diatomaceous earth and Trichoderma harzianum T-22 (Rifai Strain KRL-AG2) on the fungus gnat Bradysia sp. nr. coprophila (Diptera: Sciaridae). Journal of Economic Entomology, 100, 1353-1359.

Cloyd, R. A. (2008) Management of fungus gnats (Bradysia spp.) in greenhouse and nurseries. Floriculture and Ornamental Biotechnology, 2, 84-89.

Cloyd, R. A. \& Dickinson, A. (2008) Procedure to enhance the recovery rate of fungus gnat, Bradysia sp. nr. coprophila Lintner (Diptera: Sciaridae) adults from growing medium. HortScience, 43, 1528-1530.

Cloyd, R. A. (2009) Western flower thrips (Frankliniella occidentalis) management on ornamental crops grown in greenhouses: Have we reached an impasse. Pest Technology, 3, 1-9.

Cloyd, R. A., Marley, K. A., Larson, R. A. \& Arieli B. (2010) Bounce ${ }^{\circledR}$ fabric softener dryer sheets repel fungus gnat, Bradysia sp. nr. coprophila (Diptera: Sciaridae), Adults. Hortscience, 45, 1830-1833.

Cloyd, R. A. (2014) Horticultural Entomology/Plant Protection, Department of Entomology, Kansas State University, Manhattan, KS, USA, Personal observation.

Cloyd R. A. (2015) Ecology of fungus gnats (Bradysia spp.) in greenhouse production systems associated with disease-interactions and alternative management strategies. Insects, 6, 325332.

Cleveland, C. B., Mayes, M. A. \& Cryer, S. A. (2001) An ecological risk assessment for Spinosad use on cotton. Pest Management Science, 58, 70-84.

Dennis, D. J. (1978) Observations of fungus gnat damage to glasshouse cucurbits. New Zealand Journal of Experimental Agriculture, 6, 83-84.

Dobrindt, L. (2013) INBIOSOIL Progress Report: Use of semiochemicals and entomopathogenic fungi for the biological control of fungus gnats.

El-Sayed, A. M., Suckling, D. M., Byers, J. A., Jang, E. B. \& Wearing, C. H. (2009) Potential of 'lure and kill' in long-term pest management and eradication of invasive species. Journal of Economic Entomology, 102, 815-835.

Evans, M. R., Smith, J. N. \& Cloyd, R. A. (1998) Fungus gnat population development in coconut coir and Sphagnum peat-based substrate. HortTechnology, 8, 406-409. 
Fawzi, T. H. \& Kelly, W. C. (1982) Cavity spot of carrots caused by feeding of fungus gnat larvae. Journal of American Society of Horticultural Science, 107, 1177-1181.

Filotas, M., Castrillo, L., Vandenberg, J., Sanderson, J. \& Wraight, S. (2005) Novel isolates of the entomopathogenic fungus Beauveria bassiana for biolpogical controlp of the shore fly, Scatella tenicosta. IOBC/WPRS bulletin, 28, 95-98.

Gardiner, R. B., Jarvis, W. R. \& Shipp, J. L. (1990) Ingestion of Pythium spp. by larvae of fungus gnat Bradysia impatiens (Diptera: Sciaridae). Annals of Applied Biology, 116, 205-212.

Gillespie, D. R. \& Quiring, D. M. J. (1990) Biological control of fungus gnats, Bradysia spp. (Diptera: Sciaridae), and western flower thrips, Frankliniella occidentalis (Pergande) (Thysanoptera: Thripidae), in greenhouses using a soil-dwelling predatory mite, Geolaelaps sp. nr. aculeifer (Canestrini) (Acari: Laelapidae), Canadian Entomologist, 122, 975-983.

Gillespie, D. R. \& Menzies, J. G. (1993) Fungus gnats vector Fusarium oxysporum f. sp. Radicislycopersici. Annals of Applied Biology, 123, 539-544.

Hajek, A. E. \& St. Leger, R. J. (1994) Interactions between fungal pathogens and insect hosts. Annual Review of Entomology, 39, 293-322.

Hamlen, R. A. \& Mead, F. W. (1979) Fungus gnat larval control in greenhouse plant production. Journal of Economic Entomology, 72, 269-271.

Höfte, H. \& Whiteley, H. R. (1989) Insecticidal crystal proteins of Bacillus thuringiensis, Microbiological Reviews, 53, 242-255.

Harris, M. A., Oetting, R. D. \& Gardner, W. A. (1995) Use of entomopathogenic nematodes and a new monitoring technique for control of fungus gnats, Bradysia Coprophila (Diptera: Sciaridae), in floriculture. Biological Control, 5, 412-418.

Harris, M. A., Gardner, W. A. \& Oetting, R. D. (1996) A review of the scientific literature on fungus gnats (Diptera: Sciaridae) in the genus Bradysia. Journal of Entomological Science, 31, 252271.

Heuskin, S., Lorge, S., Godin, B., Leroy, P., Frère, I., Verheggen, F. J., Haubruge, E., Wathelet, J. P., Mestdagh, M., Hance, T. \& Lognay, G. (2012) Optimisation of a semiochemical slow-release alginate formulation attractive towards Aphidius ervi Haliday parasitoids. Pest management science, 68, 127-136.

Hungerford, H. B. (1916) Sciara maggots injurious to potted plants. Journal of Economic Entomology, 9, 538-549. 
Jackson, M. A., Dunlap, C. A. \& Jaronski, S. T. (2010) Ecological considerations in producing and formulating fungal entomopathogens for use in insect biocontrol. BioControl, 55, 129-145.

Jagdale, G. B., Casey, M. L., Grewal, P. S. \& Lindquist, R. K. (2004) Application rate and timing, potting medium, and host plant effects on the efficacy of Steinernema feltiae against the fungus gnat, Bradysia coprophila, in floriculture. Biological Control, 29, 296-305.

James, R. L., Dumroese, R. K. \& Wenny, D. L. (1995) Botrytis cinerea carried by adult fungus gnats (Diptera: Sciaridae) in container nurseries. Tree Planters' Notes, 46, 48-53.

Jess, S. \& Bingham, J. F. W. (2004) Biological control of sciarid and phorid pests of mushroom with predatory mites from the genus Hypoaspis (Acari: Hypoaspidae) and the entomopathogenic nematode Steinernema feltiae. Bulletin of entomological research, 94, 159-167.

Jiang, Y. \& Mulla, M. S. (2006) Susceptibility of the adult eye gnat Liohippelates collusor (Diptera: Chloropidae) to neonicotinoids and spinosad insecticides. Journal of Vector Ecology, 31, 6570.

Kennedy, M. K. (1974) Survival and development of Bradysia impatiens (Diptera: Sciaridae) on fungal and non-fungal food sources. Annals of the Entomological Society of America, 67, 745749.

Koller, M. (2011) Comparison of steinernema feltiae, bacillus thuringiensis israelenis and azadirachtin to control sciarids in organic potted herbs. Acta horticulturae, 915, 179-184.

Lacey, L. A., Frutos, R., Kaya, H. K. \& Vail, P. (2001) Insect pathogens as biological control agents: do they have a future? Biological Control, 21, 230-248.

Lindquist, R. K., Faber, W. R. \& Casey, M. L. (1985) Effect of various soilless root media and insecticides on fungus gnats. HortScience, 20, 358-360.

Ludwig, S. W. \& Oetting, R. D. (2001) Evaluation of medium treatments for management of Frankliniella occidentalis (Thripidae: Thysanoptera) and Bradysia coprophila (Diptera: Sciaridae). Pest Management Science, 57, 1114-1118.

Margalit, J., Lahkim-Tsror, L., Pascar-Gluzman, C. \& Bobroglo, H. (1985) Biocontrol of mosquitoes in Israel. In: Laird, M. \& Miles, J. W. (eds.) Integrated mosquito control methodologies. Academic Press, 361-374.

Meers, T. L. \& Cloyd, R. A. (2005) Egg-Laying preference of female fungus gnat Bradysia sp. nr. coprophila (Diptera: Sciaridae) on three different soilless substrates. Journal of Economic Entomology, 98, 1937-1942. 
Mertz, F. P. \& Yao, R. C. (1990) Saccharopolyspora spinosa sp. nov. isolated from soil collected in a sugar rum still. International Journal of Systematic Bacteriology, 40, 34-39.

Molloy, D., Gaugler, R. \& Jamnback, H. (1981) Factors influencing efficacy of Bacillus thuringiensis var. israelensis as a biological control agent of black fly larvae. Journal of Economic Entomology, 74, 61-64.

Mordue, A. J. \& Nisbet, A. J. (2000) Azadirachtin from the neem tree Azadirachta indica: its action against insects. Anais da Sociedade Entomológica do Brasil, 29, 615-632.

Narusuye, K., Kawai, F., Matsuzaki, K. \& Miyachi, E. (2005) Linalool suppresses voltage-gated currents in sensory neurons and cerebellar purkinije cells. Journal of neural transmission, 112, 193-203.

Olson, D. L., Oetting, R. D. \& van lersel, M. W. (2002) Effect of soilless potting media and water management on development of fungus gnats (Diptera: Sciaridae) and plant growth. HortScience, 37, 919-923.

Osborne, L. S., Boucias, D. G. \& Lindquist, R. K. (1985) Activity of Bacillus thuringiensis var. israelensis on Bradysia coprophila (Diptera: Sciaridae). Journal of Economic Entomology, 78, 922-925.

Orr, N., Shaffner, A. J., Richey, K. \& Crouse, G. D. (2009) Novel mode of action of Spinosad: receptor binding studies demonstrating lack of interaction with known insecticide target sites. Pesticide Biochemistry and Physiology, 95, 1-5.

Pedrini, N. (2018) Molecular interactions between entomopathogenic fungi (Hypocreales) and their insect host: Perspectives from stressful cuticle and hemolymph battlefields and the potential of dual RNA sequencing for future studies. Fungal Biology, 122, 538-545.

Perez, C. M., Marina, C. F., Bond, J. G., Rojas, J. C., Valle, J. \& Williams, T. (2007) Spinosad, a naturally derived insecticide, for control of Aedes aegypti (Diptera: Culicidae): efficacy, persistence, and elicited oviposition response. Journal of Medical Entomology, 44, 631-638.

Quesada-Moraga, E., Carrasco-Dı'az, J. A. \& Santiago-A' Ivarez, C. (2006) Insecticidal and antifeedant activities of proteins secreted by entomopathogenic fungi against Spodoptera littoralis (Lep., Noctuidae). Journal of Applied Entomology, 130, 442-452.

Quintela, E. D. \& McCoy, C. W. (1998) Synergistic effect of imidacloprid and two Entomopathogenic fungi on the behavior and survival of larvae of Diaprepes abbreviatus (Coleoptera: Curculionidae) in soil. Journal of Economic Entomology, 91, 110-122. 
Raudenbush, A. L., Cloyd, R. A. \& Echegaray, E. R. (2014) Effect of physical barrier on adult emergence and egg survival associated with the fungus gnat, Bradysia sp. nr. coprophila (Diptera: Sciaridae), under laboratory conditions. HortScience, 49, 905-910.

Roberts, D. W., Gupta, S. \& St. Leger, R. J. (1992) Metabolite production by entomopathogenic fungi. Pesquisa Agropecuária Brasileira, 27, 325-347.

Salgado, V. L. (1998) Studies on the mode of action of spinosad: insect symptoms and physiological correlates. Pesticide Biochemistry and Physiology, 60, 91-102.

Scott, J. G. (1998) Toxicity of spinosad to susceptible and resistant strains of house flies, Musca domestica. Pesticide science, 54, 131-133.

Stark, J. D., Vargas, R. \& Miller, N. (2004) Toxicity of spinosad in protein bait to three economically important Tephritid fruit fly species (Diptera: Tephritidae) and their parasitoids (Hymenoptera: Braconidae). Journal of Economic Entomology, 97, 911-915.

Stanghellini, M. E. \& El-Hamalawi, Z. A. (2005) Efficacy of Beauveria bassiana on colonized millet seed as a biopesticide for the control of shore flies. HortScience, 40, 1384-1388.

Steffan, W.A. (1966) A generic revision of the family Sciaridae (Diptera) of America north of Mexico. University of California Publications in Entomology, 44, 1-77.

Sznyk-Basalyga, A. \& Bednarek, A. (2003) Integrated control of Lycoriella solani (Diptera: Sciaridae) with entomopathogenic nematodes and insecticides. Insect Pathogens and Insect Parasitic Nematodes IOBC wprs Bulletin, 26, 189-192.

Tavoosi Ajvad, F., Madadi, H., Michaud, J. P., Zafari, D. \& Khanjani, M. (2019) Combined applications of an entomopathogenic fungus and a predatory Mite to control fungus gnats (Diptera: Sciaridae) in mushroom production. Biological Control, 141.

Thompson, G. D., Dutton, R. \& Sparks, T. C. (2000) Spinosad - a case study: an example from a natural products discovery programme. Pest Management Science, 56, 696-702.

Toews, M. D. \& Subramanyam, B. (2003) Contribution of contact toxicity and wheat condition to mortality of stored-product insects exposed to spinosad. Pest Management Science, 59, 538544.

Trienens, M. \& Rohlfs, M. (2012) Insect-fungus interference competition - the potential role of global secondary metabolite regulation, pathway-specific mycotoxin expression and formation of oxylipins. Fungal Ecology, 5, 191-199.

Vega, F. E., Posada, F., Aime, M. C., Pava-Ripoll, M., Infante, F. \& Rehner, S. A. (2008) Entomopathogenic fungal endophytes. Biological Control, 46, 72-82. 
Vernon, R. S., Van Herk, W. G., Clodius, M. \& Tolman, J. (2015) Companion planting attract-and-kill method for wireworm management in potatoes. Journal of Pest Science, 89, 375-389.

Vey, A., Hoagland, R. \& Butt, T. M. (2001) Toxic metabolites of fungal biocontrol agents. In: Butt, T. M., Jackson, C. W. \& Magan, N. (eds.) Fungi as biocontrol agents progress, problems and potential. CABI Publishing, Wallingford, UK, 311-346.

Wilkinson, J. D. \& Daugherty, D. M. (1970a) The biology and immature stages of Bradysia impatiens (Diptera: Sciaridae). Annals of the Entomological Society of America, 63, 656-660.

Yee, W. L. (2018) Spinosad versus spinetoram effects on kill and oviposition of Rhagoletis indifferens (Diptera: Tephritidae) at differing fly ages and temperatures. Journal of Insect Science, 18. 


\section{Publications}

\section{Publications}

\section{Presentations at conferences}

Dehghani, M., Rademacher, J., Vidal, S. (2019) Laboratory evaluation of combining an entomopathogenic fungus (EPF) and a botanical compound to control fungus gnats, Bradysia spp., International Congress on Invertebrate Pathology and Microbial Control \& 52nd Annual Meeting of the Society for Invertebrate Pathology \& $17^{\text {th }}$ Meeting of the IOBC-WPRS Working Group “Microbial and Nematode Control of Invertebrate Pests, Valencia, Spain.

Dehghani, M., Rademacher, J., Vidal, S. (2019) Effect of combining an entomopathogenic fungus (EPF) and a botanical compound on attraction and oviposition of the fungus gnat, Bradysia spp. DGaaE, Entomologentagung, Halle (Saale), Germany.

Dehghani, M., Rademacher, J., Vidal, S. (2018) An attract and kill formulation for fungus gnat control combining an entomopathogenic fungal strain and a botanical compound. 36 . Tagung des DPG-Arbeitskreises Nutzarthropoden und Entomopathogene Nematoden, Bremen, Germany.

Dehghani, M., Vidal, S. (2018) Combining entomopathogenic fungal isolates and botanical compounds for control of fungus gnat larvae (Diptera: Sciaridae). XI European Congress of Entomology, Naples, Italy.

\section{Poster at conference}

Dehghani, M., Vidal, S. (2017). The influence of different entomopathogenic fungi (EPF) on fungus gnat larvae. DGaaE, Entomologentagung, Freising, Germany. 


\section{Acknowledgements / Danksagung}

Ein Teil der Gruppe Agrarentomologie in Göttingen zu werden, verdanke ich meinen Supervisor Herrn Prof. Dr. Stefan Vidal. Ihm danke ich sehr viel für die Unterstützung bei diesen interessanten und praxisorientierten Thema, den mir gegebenen Freiraum für eigene Ideen, der Möglichkeit an nationalen und internationalen Konferenzen teilzunehmen sowie der Durchsicht der Manuskripte. Ich danke ihm für die sehr gute und wertvolle Zusammenarbeit, der vielen interessanten Gespräche im Büro, Ideen und Hilfestellungen.

Ich möchte mich besonders bei Herrn Dr. sc. nat. Rainer Meyhöfer und Herrn Prof. Dr. Petr Karlovsky für Ihre Bereitschaft und für die Mitgliedschaft in meinem Prüfungskomitee bedanken.

Dank gilt dem gesamten Team der Katz Biotech AG für die gute Zusammenarbeit. Ich danke im Besonderen Herrn Jörg Rademacher für die Bereitstellung der Kapseln für meine Versuche und den Austausch unserer Erfahrungen.

Mein Dank gilt ebenfalls Frau Bianca Tappe für ihre unermüdliche Hilfe und der vielen lehrreichen Gespräche.

Ich danke meinen Kollegen und Freunden, welche mich neben dem Studium begleitet haben und in unterschiedlichster Weise helfend zur Seite standen: Dr. Michael Albert Brandl, Dr. Khaldon Askoul, Dr. Laurenz Hettlage, Nongcebo Sandra Memela und Hadis Jayanti.

Allen anderen Kollegen und Kolleginnen der Agrarentomologie danke ich ebenfalls für die offene und freundliche Zusammenarbeit.

Ein herzlicher Dank gilt unserem Technikerteam: vor allem Maik Knobel und Frank Gremmes.

Zum Schluss bedanke ich mich neben meiner Familie und meinen Freunden bei zwei besonderen Menschen, welche mich vor und während meines Studiums begleitet haben. Zum einen bei Dr. med. Farokhzad Baharestani, der mich bei meinen Schritten in Deutschland geholfen hat. Und zum Schluss meinen Ehemann Tobias, welcher mich stets motiviert und unterstützt hat.

Vielen Dank! 


\section{Erklärungen}

1. Hiermit erkläre ich, dass diese Arbeit weder in gleicher noch in ähnlicher Form bereits anderen Prüfungsbehörden vorgelegen hat.

Weiter erkläre ich, dass ich mich an keiner anderen Hochschule um einen Doktorgrad beworben habe.

Göttingen, den

(Unterschrift)

2. Hiermit erkläre ich eidesstattlich, dass diese Dissertation selbständig und ohne unerlaubte Hilfe angefertigt wurde.

Göttingen, den

(Unterschrift) 